\title{
MEDIUM ACCESS USING QUEUES
}

\author{
By Devavrat Shah ${ }^{\S}$, Jinwoo Shin ${ }^{\llbracket}$ and Prasad Tetali ${ }^{\|}$ \\ MIT $T^{\S}$ IBM Research and Georgia Tech ${ }^{\|}$
}

\begin{abstract}
Consider a wireless network of $n$ nodes represented by a graph $G=$ $(V, E)$ where an edge $(i, j) \in E$ models the fact that transmissions of $i$ and $j$ interfere with each other, i.e. simultaneous transmissions of $i$ and $j$ become unsuccessful. Hence it is required that at each time instance a set of non-interfering nodes (corresponding to an independent set in $G$ ) access the wireless medium. To utilize wireless resources efficiently, it is required to arbitrate the access of medium among interfering nodes properly. Moreover, to be of practical use, such a mechanism is required to be fully distributed as well as simple.

As the main result of this paper, we provide such a medium access algorithm. It is randomized, fully distributed and simple: each node attempts to access medium at each time with probability that is a function of its local information. We establish its efficiency by showing that the corresponding network Markov chain is positive recurrent as long as the demand imposed on the network can be supported by the wireless network (using any algorithm). In that sense, the proposed algorithm is optimal in terms of utilizing wireless resources. The algorithm is oblivious to the network graph structure, in contrast with the so-called polynomial back-off algorithm that was established to be optimal only for a certain class of graphs. The key methodological innovations are (a) establishing the positive recurrence of coupled Markov chains, and (b) a comparison relation between stationary distributions of Markov chains building upon the classical Markov chain tree theorem.
\end{abstract}

1. Introduction. We consider a single-hop wireless network of $n$ nodes or queues represented by $V=\{1, \ldots, n\}$. Time is discrete indexed by $\tau \in$ $\{0,1, \ldots\}$. Unit-size packets arrive at queue $i$ as per an exogenous process.

${ }^{\S}$ D. Shah is with the Department of Electrical Engineering and Computer Science at Massachusetts Institute of Technology, Cambridge, MA 02139 USA. The author's email address: devavrat@mit.edu

ฯ J. Shin is with the Mathematical Sciences Department at IBM T. J. Watson Research, Yorktown Heights, NY 10598 USA. The author's email address: jshin@us.ibm.com

${ }^{\|} \mathrm{P}$. Tetali is with the School of Mathematics and the School of Computer Science at Georgia Institute of Technology, Atlanta, GA 30332 USA. The author's email address: tetali@math.gatech.edu

AMS 2000 subject classifications: Primary 60K20, 68M12; Secondary 68M20

Keywords and phrases: Wireless Medium Access, Positive Recurrence, Contention Resolution Protocol, Mixing Time 
Let $\zeta_{i}(\tau)$ denote the number of packets arriving at queue $i$ in the time slot $[\tau, \tau+1)$. For simplicity, we shall assume $\zeta_{i}(\cdot)$ as an independent Bernoulli process with rate $\lambda_{i}$, i.e. $\lambda_{i}=\mathbb{P}\left(\zeta_{i}(\tau)=1\right)$ and $\zeta_{i}(\tau) \in\{0,1\}$ for all $i, \tau \geq 0 .{ }^{1}$ Let $Q_{i}(\tau) \in \mathbb{N}$ be the number of packets in queue $i$ at the beginning of time slot $[\tau, \tau+1)$.

The work from queues is served at the unit rate subject to interference constraints. Specifically, let $G=(V, E)$ denote the inference graph $:(i, j) \in$ $E$ implies that queues $i$ and $j$ can not transmit simultaneously since their transmissions interfere with each other. Formally, let $\sigma_{i}(\tau) \in\{0,1\}$ denote whether the queue $i$ is (successfully) transmitting at time $\tau$, and let $\boldsymbol{\sigma}(\tau)=$ $\left[\sigma_{i}(\tau)\right]$. Then,

$\boldsymbol{\sigma}(\tau) \in \mathcal{I}(G) \triangleq\left\{\boldsymbol{\rho}=\left[\rho_{i}\right] \in\{0,1\}^{n}: \rho_{i}+\rho_{j} \leq 1\right.$ for all $\left.(i, j) \in E\right\}, \quad$ for $\tau \geq 0$,

i.e. $\mathcal{I}(G)$ is the set of independent sets of $G$. The resulting queueing dynamics can be summarized as

$$
Q_{i}(\tau+1)=Q_{i}(\tau)-\sigma_{i}(\tau) \mathbf{I}_{\left\{Q_{i}(\tau)>0\right\}}+\zeta_{i}(\tau), \text { for } \tau \geq 0,1 \leq i \leq n .
$$

Here $\mathbf{I}_{\{x\}}=1$ if $x=$ 'true' and 0 if $x=$ 'false'.

Now an algorithm, which we shall call medium access, is required to choose $\boldsymbol{\sigma}(\tau) \in \mathcal{I}(G)$ at the beginning of each time $\tau$. A good medium access algorithm should choose $\boldsymbol{\sigma}(\tau)$ so as to utilize the wireless medium as efficiently as possible. Putting it another way, it should be able to keep queues finite for as large a set of arrival rates $\boldsymbol{\lambda}=\left[\lambda_{i}\right]$ as possible. Towards this, define the capacity region

$$
\boldsymbol{\Lambda}=\left\{\boldsymbol{y} \in \mathbb{R}_{+}^{n}: \boldsymbol{y}<\sum_{\boldsymbol{\sigma} \in \mathcal{I}(G)} \alpha_{\boldsymbol{\sigma}} \boldsymbol{\sigma} \text { with } \alpha_{\boldsymbol{\sigma}} \geq 0, \sum_{\boldsymbol{\sigma} \in \mathcal{I}(G)} \alpha_{\boldsymbol{\sigma}} \leq 1\right\}
$$

Since $\boldsymbol{\sigma}(\tau) \in \mathcal{I}(G)$, the effective 'service' rate induced by any algorithm over time is essentially in the closure of $\boldsymbol{\Lambda}$. Therefore, a medium access algorithm can be considered optimal, if it can keep queues finite, for any $\boldsymbol{\lambda} \in \boldsymbol{\Lambda}$. Formally, if the state of the queueing system including the algorithm's decisions and queue-sizes can be described as a Markov chain, then the existence of a stationary distribution for this Markov chain and its ergodicity effectively

\footnotetext{
${ }^{1}$ This Bernoulli assumption does not hurt the generality of this paper since there is a simple algorithmic trick to justify this. For any non-Bernoulli (even adversarial) arrival process with rate $\lambda_{i}$, store packets in a virtual 'gate-keeper' queue and output packets as per a Bernoulli process with rate $\lambda_{i}+\varepsilon$. Then, the arrival dynamics of packets seen by the network becomes a Bernoulli(-like) process. The result of this paper is easily extendable for non-Bernoulli arrival processes using the algorithmic trick.
} 
implies that the queues remain finite. A sufficient condition for this is aperiodicity and positive recurrence of the corresponding Markov chain. This motivates the following (first-order) performance metric for medium access algorithms.

Definition 1 (Optimal) A medium access algorithm is called optimal ${ }^{2}$ if for any $\boldsymbol{\lambda} \in \boldsymbol{\Lambda}$ the (appropriately defined) underlying network Markov chain is positive recurrent and aperiodic.

To be of practical use, the medium access algorithm ought to be simple and fully distributed, i.e. should use only local information like queue-size, and past collision history. In such an algorithm, each node makes the decision to transmit or not on its own, at the beginning of each time slot. At the end of the time slot, it learns whether attempted transmission was successful or not (due to a simultaneous attempt of transmission by a neighbor). If a node does not transmit, then it learns whether any of its neighbors attempted transmission. And, ideally such an algorithm should be optimal.

1.1. Prior Work. Design of an efficient and distributed medium access algorithm has been of interest since the ALOHA algorithm for the radio network [1] and Local Area Networks [15] in the 1970s. Subsequently a variety of the so-called back-off algorithms or similar protocols have been extensively studied. Various negative and positive properties of back-off protocols were established in various works $[12,17,13,23,2,14]$.

Specifically, Hastad, Leighton and Rogoff [9] studied a medium access algorithm in which each node or queue attempts transmission at each time with probability that is inversely proportional to a polynomial function of the number of successive failures in the most recent past. They established it to be optimal when the interference graph $G$ is complete, or equivalently all nodes are competing for one resource (as in the classical Ethernet/LAN). The optimality of this polynomial back-off algorithm was further established when it is induced by matching constraints in a bipartite graph $G$ by Goldberg and MacKenzie [7]. However, the optimality of polynomial back-off or any other fully distributed medium access algorithm remained open for general graphs. The interested reader may find a summary of results until 2001, on medium access on a webpage maintained by Goldberg [8].

In the past year or so, significant progress has been made towards this open question, i.e., designing a distributed optimal medium access algorithm for general graphs. Specifically, Rajagopalan, Shah and Shin (RSS) [18, 19] and Jiang and Walrand (JW) [11] proposed two different medium access

\footnotetext{
${ }^{2}$ Equivalently, it is also called 'throughput-optimal' or 'stability-optimal'.
} 
algorithms that operate in continuous time. The RSS algorithm is optimal but requires a bit of information exchange between each pair of neighboring nodes per unit time. The JW algorithm was established to have a weaker form of optimality, called 'rate stability', by Jiang, Shah, Shin and Walrand [10]. In summary, both algorithms stop short of being fully distributed and optimal. More importantly, both of them operate in continuous time (which makes analysis much easier though) and thus effectively avoiding the issue of loss in performance due to contention present in the discrete time setup that considered in this paper as well as most works studying back-off algorithms.

1.2. Our Contribution. The main result of this paper is a fully distributed medium access algorithm that is optimal for any interference graph $G$. It resolves an important question in distributed computation that has been of great practical interest. The proposed medium access algorithm builds on the RSS algorithm and overcomes its two key limitations by adapting it to the discrete time setup and removing the need for any information exchange between neighboring nodes. In what follows, we explain in detail how we overcome such limitations.

In the proposed medium access algorithm, each node attempts transmission in each time slot based on: (a) whether it managed to successfully transmit in the previous time slot, or whether any of its neighbors attempted to transmit in the previous time slot; (b) local queue-size and estimation of the "weight" of the neighbors. Given this information, each node in the beginning of each time slot attempts transmission with probability depending upon (a) and (b). Specifically, if the node was successful in the previous time, it does not transmit at this time with probability that is inversely proportional to its own weight that depends on (b). Else if no other neighbor attempted transmission in the previous time then a node attempts transmission with probability $\frac{1}{2}$. Otherwise, with probability 1 , a node does not transmit.

In such an algorithm, the only seeming non-local information is the estimation of the neighbors' weight in (b). An important contribution of this work is the design of a non-trivial learning mechanism, based only on information of type (a), that estimates the neighbors' weight without any explicit information exchange. We note that, in contrast, the RSS algorithm had required explicit information exchange for estimating the neighbors' weight.

To establish optimality of the proposed algorithm, we show that, in essence, $\sum_{i} \sigma_{i}(\tau) \log \log Q_{i}(\tau)$ is close to $\max _{\boldsymbol{\rho} \in \mathcal{I}(G)} \sum_{i} \rho_{i} \log \log Q_{i}(\tau)$, on average for all large enough $\tau$. That is, effectively the distributed medium access chooses $\sigma(\tau)$ that is (close to) maximum weight independent set of $G$ when 
node weights are equal to $\log \log$ of the queue-sizes. Such a property is known (cf. $[20,22]$ ) to imply that $\sum_{i} F\left(Q_{i}(\tau)\right.$ ) (where $F(x)=\int_{0}^{x} \log \log y d y$ ) is a potential (or Lyapunov, energy) function for the system state so that the function is expected to decrease by at least a fixed amount as long as $\boldsymbol{\lambda} \in \boldsymbol{\Lambda}$. This subsequently establishes that the network as a Markov chain is positive recurrent (implying the optimality of the algorithm).

We establish the near optimality of $\sum_{i} \sigma_{i}(\tau) \log \log Q_{i}(\tau)$ under the medium access algorithm in two steps. To begin with, we observe that the evolution of $\boldsymbol{\sigma}(\tau)$ under the algorithm is a Markov chain on the space of independent sets $\mathcal{I}(G)$ with time-varying transition probabilities. For this Markov chain, at any particular time $\tau$, let $\boldsymbol{\pi}(\tau)$ be the stationary distribution at time $\tau$ (given transition probabilities at time $\tau$ ).

In the first step, we study this (time-varying, stationary) distribution $\boldsymbol{\pi}(\tau)$ and show that it is approximately 'product-form'. To obtain such an approximate characterization, we show that the transition probabilities of the Markov chain are well approximated by those of a reversible Markov chain which has a product-form stationary distribution. A novel comparison relation between stationary distributions of two Markov chains in terms of the relation between their transition probabilities leads to the approximate product-form characterization of $\boldsymbol{\pi}(\tau)$. We note that the RSS algorithm (and similarly, the JW algorithm) had used the continuous time setting to make sure that the corresponding Markov chain was reversible and hence had a product-form distribution to start with; such reversibility is lost in general in the discrete time setting of this paper. Using this approximate product-form characterization of $\boldsymbol{\pi}(\tau)$ in addition to the Gibbs' maximal principle (cf. [6]), we prove that $\boldsymbol{\pi}(\tau)$ has the desired property; namely, that $\sum_{i} \sigma_{i} \log \log Q_{i}(\tau)$ is close to $\max _{\boldsymbol{\rho} \in \mathcal{I}(G)} \sum_{i} \rho_{i} \log \log Q_{i}(\tau)$ if $\boldsymbol{\sigma}=\left[\sigma_{i}\right]$ is given by the distribution $\boldsymbol{\pi}(\tau)$. We call this the maximum-weight property at stationarity.

In the second step, we show that the Markov chain, despite it being timevarying, is always near stationarity for large enough $\tau$ by carefully estimating the effective mixing time of the time-varying Markov chain. In other words, the distribution of $\boldsymbol{\sigma}(\tau)$ is close to $\boldsymbol{\pi}(\tau)$ for large enough $\tau$. Therefore, the maximum-weight property at stationarity (established in the first step) implies that $\sum_{i} \sigma_{i}(\tau) \log \log Q_{i}(\tau)$ is close to $\max _{\boldsymbol{\rho} \in \mathcal{I}(G)} \sum_{i} \rho_{i} \log \log Q_{i}(\tau)$. To guarantee the near stationarity property as a consequence of such a mixing analysis, it is required that a design of 'weight' maintained by each node in the medium access algorithm utilizes the neighbor's weight information. As mentioned earlier, we resolve this by developing a learning mechanism that estimates the neighbor's weight based on the information whether it trans- 
mitted or not thus far. The learning mechanism is a recursive updating rule to produce a statistically optimal estimate of the underlying system state. The success in this second step is primarily due to our novel (and highly non-trivial) choice of parameters in the learning mechanism to incorporate well with the mixing time analysis of the time-varying Markov chain.

Our results have two implicit methodological contributions. First is the method (or criterion) to establish positive recurrence of two coupled Markov chains when both components on their own are known to be positive recurrent and have complicated coupling. Second is the general comparison relation between stationary distributions of two finite state Markov chains building upon the classical Markov chain tree theorem.

1.3. Organization. The remainder of the paper is organized as follows. Section 2 presents formally the medium access algorithm and a statement of the main result. Section 3 summarizes our proof strategy for the main result at a high level. Section 4 presents necessary preliminaries that are used to establish the main result in Section 5. Section 6 and 7 are for providing the detailed proof of the main lemma in Section 5 .

2. Algorithm and Its Optimality. The medium access algorithm is randomized, distributed, simple and runs in discrete time with time indexed by $\tau \geq 0$. Each node $i \in V$ maintains weight $W_{i}(\tau) \in \mathbb{R}_{+}$over $\tau \geq 0$. In the beginning of each time slot $\tau \geq 0$, each node $i \in V$ decides to attempt transmission or not as follows:

1. If the transmission of node $i$ was successful at $\tau-1$, then

$\circ$ it attempts to transmit with probability $1-\frac{1}{W_{i}(\tau)}$.

2. Else if no neighbor of $i$ attempted transmission at $\tau-1$, then

$\circ$ it attempts to transmit with probability $\frac{1}{2}$.

3. Otherwise, it does not attempt to transmit with probability 1.

Now we describe how each node $i$ maintains weight $W_{i}(\tau)$ :

$$
W_{i}(\tau)=\max \left\{\log Q_{i}(\tau), \max _{j \in \mathcal{N}(i)} \exp \left(\sqrt{\log g\left(A_{j}^{i}(\tau)\right)}\right)\right\}
$$

where by $\log$ and $\log \log$ we mean $[\log ]_{+}$and $[\log \log ]_{+}$respectively; $g: \mathbb{R}_{+} \rightarrow$ $\mathbb{R}_{+}$is defined as $g(x)=\exp \left(\log \log ^{4} x\right)$; by $\log \log ^{4} x$ we mean $(\log \log x)^{4}$, $\log$ represents $\log _{e}$; and $\mathcal{N}(i)=\{j \in V:(i, j) \in E\}$ represents neighbors 
of node $i$. Note that $W_{i}(\tau) \geq 1$ for all $\tau$ by definition. In above, $A_{j}^{i}(\cdot)$ is a 'counter' maintained by node $i$ as a 'long term' estimate of weight $W_{j}(\cdot)$. This is maintained along with another 'counter' $B_{j}^{i}(\cdot)$ by node $i$ as a 'short term' estimate of $W_{j}(\cdot)$. Initially, $A_{j}^{i}(0)=B_{j}^{i}(0)=0$ for all $j \in \mathcal{N}(i)$ and $i \in V$. For each $j \in \mathcal{N}(i), A_{j}^{i}(\cdot)$ and $B_{j}^{i}(\cdot)$ are updated by node $i$ at $\tau$ as follows:

1. If $j \in \mathcal{N}(i)$ attempted transmission at $\tau-1$, then

$$
\circ A_{j}^{i}(\tau)=A_{j}^{i}(\tau-1) \text { and } B_{j}^{i}(\tau)=B_{j}^{i}(\tau-1)+1 .
$$

2. Else if $B_{j}^{i}(\tau-1) \geq 2$, then

$$
\begin{aligned}
\circ A_{j}^{i}(\tau) & =\left\{\begin{array}{ll}
A_{j}^{i}(\tau-1)+1 & \text { if } B_{j}^{i}(\tau-1) \geq g\left(A_{j}^{i}(\tau-1)\right) \\
A_{j}^{i}(\tau-1)-1 & \text { otherwise }
\end{array}\right. \text { and reset } \\
B_{j}^{i}(\tau) & =0 .
\end{aligned}
$$

3. Otherwise, $A_{j}^{i}(\tau)=A_{j}^{i}(\tau-1)$ and $B_{j}^{i}(\tau)=0$.

Observe that $B_{j}^{i}(\cdot)$ counts how long neighbor $j$ keeps attempting transmission consecutively. When $j$ 's transmissions are successful, the random period of consecutive transmissions is essentially distributed as per the geometric distribution with mean $W_{j}(\cdot)$ due to the nature of our algorithm. Thus $B_{j}^{i}(\cdot)$ provides a short-term (or instantaneous) estimation of $W_{j}(\cdot)$. To extract a robust estimation of $W_{j}(\cdot)$ from such short-term estimates, the long-term estimation $A_{j}^{i}(\cdot)$ is maintained: it changes by \pm 1 using $B_{j}^{i}(\cdot)$ at most once per unit time. Specifically, as per the above updates, $g\left(A_{j}^{i}(\cdot)\right)$ is acting as an estimation of $W_{j}(\cdot)$. Now it is important to note that the choice of $g$ (defined above) plays a crucial role in the quality of estimate of $W_{j}(\cdot)$. The change in estimation $g\left(A_{j}^{i}(\cdot)\right)$, when $A_{j}^{i}(\cdot)$ is updated by \pm 1 , is roughly $g^{\prime}\left(A_{j}^{i}(\cdot)\right)$. Since $W_{j}(\cdot)$ is changing over time, it is important to have $g^{\prime}(\cdot)$ not too small. On the other hand, if it is too large then it is too sensitive and could be noisy just like $B_{j}^{i}(\cdot)$. A priori it is not clear if there exists a choice of any function $g$ that allows for keeping $A_{j}^{i}(\cdot)$ for a good enough estimation of $W_{j}^{i}(\cdot)$, which subsequently leads to positive-recurrence of the network Markov chain. Somewhat surprisingly (at least to us), we find that indeed such a $g$ exists and is as defined above: $g(x)=\exp \left(\log \log ^{4} x\right)$. As per our proof technique, $g(x)=\exp \left(\log \log ^{\alpha} x\right)$ works for any $\alpha>2$; however we shall stick to the choice of $\alpha=4$ in the paper. Now we are ready to state the main result of this paper. 
Theorem 1 The medium access algorithm as described above is optimal for any interference graph.

The nature of the proof of Theorem 1 is intrinsically technical along with numerous detailed calculations. In particular, our non-trivial choice of function $g(x)=\exp \left(\log \log ^{\alpha} x\right)$, which is a key for Theorem 1 , is driven by a serious of detailed calculations and lemmas, rather than a simple reasoning. In the following section, we provide the technical reasons at a high level for such a choice of function $g$.

3. Proof of Theorem 1: An Overview. This section provides an overview of the proof of Theorem 1 to explain the key challenges involved in establishing it as well as intuition behind the particular choice of function $g$. The goal in this section is not to provide precise arguments but only provide intuition so as to assist a reader in understanding the structure of the proof. The complete proof with all details is stated in Sections 5, 6 and 7 .

Theorem 1 requires establishing positive recurrence of an appropriate Markov chain that describes the evolution of the network state under the medium access algorithm described. To that end, define

$$
X(\tau)=(\mathbf{Q}(\tau), \boldsymbol{\sigma}(\tau), \boldsymbol{a}(\tau), \boldsymbol{A}(\tau), \mathbf{B}(\tau))
$$

where $\mathbf{Q}(\tau)$ represents vector of queue-sizes; $\boldsymbol{a}(\tau) \in\{0,1\}^{n}$ denotes the vector of transmission attempts by nodes at time $\tau ; \boldsymbol{\sigma}(\tau) \in \mathcal{I}(G)$ denotes the vector of resulting successful transmissions in time $\tau$ (clearly, $\boldsymbol{\sigma}(\tau) \leq \boldsymbol{a}(\tau)$ ); and $\boldsymbol{A}(\tau), \mathbf{B}(\tau) \in \mathbb{Z}_{+}^{2|E|}$ denote the vector of long-term and short-term estimations maintained at nodes as explained in Section 2. Then it follows that under medium access algorithm $X(\cdot)$ is a Markov chain.

Now a generic method to establish positive-recurrence of a Markov chain involves establishing that certain real-valued function over the state-space of the Markov chain is Lyapunov or Potential function for the Markov chain. Roughly speaking, this involves establishing that on average the value of this function decreases under the dynamics of the Markov chain if its value is high enough; Theorem 5 states the precise conditions that need to be verified. With this eventual goal, we consider the following function that maps state $\mathrm{x}=(\mathbf{Q}, \boldsymbol{\sigma}, \boldsymbol{a}, \boldsymbol{A}, \mathbf{B})$ to non-negative real values as

$$
L(\mathrm{x})=\sum_{i} F\left(Q_{i}\right)+\sum_{i ; j \in \mathcal{N}(i)}\left(\left(A_{j}^{i}\right)^{2}+g^{(-1)}\left(B_{j}^{i}\right)\right),
$$

where $F(x)=\int_{0}^{x} \log \log y d y$ with $\log \log y=[\log \log y]_{+}$; the inverse function of $g(x)=\exp \left(\log \log ^{4} x\right)$ is $g^{(-1)}(x)=\exp \left(\exp \left(\log ^{1 / 4} x\right)\right)$. With abuse 
of notation, we shall use $L(\tau)$ to denote $L(X(\tau))$. Now

$$
L(\tau)=L^{Q}(\tau)+L^{A, B}(\tau)
$$

where

$L^{Q}(\tau)=\sum_{i} F\left(Q_{i}(\tau)\right)$ and $L^{A, B}(\tau)=\sum_{i ; j \in \mathcal{N}(i)}\left(\left(A_{j}^{i}(\tau)\right)^{2}+g^{(-1)}\left(B_{j}^{i}(\tau)\right)\right)$.

The proof is devoted to establish the negative-drift property of $L(\cdot)$, i.e. if $X(\tau)=\mathrm{x}$ is such that $L(\tau)$ is large enough (i.e. larger than some finite constant), then value of $L(\cdot)$ decreases enough on average. This property is established by considering two separate cases.

Case One. When $L(\tau)$ is large due to $L^{A, B}(\tau)$ being very large.

○ Formally, when $\max _{i, j}\left(g\left(A_{j}^{i}(\tau)\right), B_{j}^{i}(\tau)\right) \geq \boldsymbol{W}_{\max }^{3}(\tau)$.

Case Two. When $L(\tau)$ is large due to $L^{Q}(\tau)$ being very large.

○ Formally, when $\max _{i, j}\left(g\left(A_{j}^{i}(\tau)\right), B_{j}^{i}(\tau)\right)<\boldsymbol{W}_{\max }^{3}(\tau)$.

In above, $\boldsymbol{W}_{\max }(\tau)=\max _{i} W_{i}(\tau)$

Case One. In this case, there exists $i \in V$ and $j \in \mathcal{N}(i)$ so that $g\left(A_{j}^{i}(\tau)\right)$ or $B_{j}^{i}(\tau)$ is larger than $\boldsymbol{W}_{\max }^{3}(\tau)$. Using the property of the estimation procedure (which updates $A_{j}^{i}(\cdot)$ ), we show that the $L^{A, B}(\cdot)$ decreases on average by a large amount; it is large enough so that it dominates the possible increase in any other components of $L(\cdot)$. Such a strong property holds because as per the algorithm, $g\left(A_{j}^{i}(\tau)\right)$ and $B_{j}^{i}(\tau)$ continually try to estimate $W_{j}(\tau)$ and hence if either of them is larger than $\boldsymbol{W}_{\max }^{3}(\tau)$, they ought to decrease by a large amount in a short time. Indeed, to translate this property into sufficient decrease of $L(\cdot)$, the careful choice of $L^{A, B}(\cdot)$ is made. This case is dealt in detail in Section 6.

Case Two. In this case, for each $i \in V$ and $j \in \mathcal{N}(i), g\left(A_{j}^{i}(\tau)\right)$ and $B_{j}^{i}(\tau)$ are smaller than $\boldsymbol{W}_{\max }^{3}(\tau)$. To establish the decrease in $L(\cdot)$, we show that in this case $L^{Q}(\cdot)$ decreases by large enough amount; large enough so that it dominates the possible increase in $L^{A, B}(\cdot)$. This case crucially utilizes the property of the medium access algorithm, the choice of the weights $W_{i}(\cdot)$ for $i \in V$ and the form of function $g$. The precise details explaining how these play roles in establishing this decrease in $L^{Q}(\cdot)$ are provided in Section 7. Here, we shall provide key ideas behind these somewhat involved arguments. 
The property that $L^{Q}(\cdot)$ decreases by large enough amount follows if we establish that the set of transmitting nodes $\boldsymbol{\sigma}(\tau)$ is such that

$$
\sum_{i} \sigma_{i}(\tau) \log \log Q_{i}(\tau) \approx \max _{\boldsymbol{\rho} \in \mathcal{I}(G)} \sum_{i} \rho_{i} \log \log Q_{i}(\tau) .
$$

See Lemma 13 for further details. To establish (3), using the condition of the second case $g\left(A_{j}^{i}(\tau)\right)<\boldsymbol{W}_{\max }^{3}(\tau)$ for all $i \in V$ and $j \in \mathcal{N}(i)$, we essentially show that

$$
\begin{aligned}
g\left(A_{j}^{i}(\tau)\right) & \approx W_{j}(\tau), \text { for all } i \in V, j \in \mathcal{N}(i), \text { and } \\
\sum_{i} \sigma_{i}(\tau) \log \log Q_{i}(\tau) & \approx \max _{\boldsymbol{\rho} \in \mathcal{I}(G)} \sum_{i} \rho_{i} \log W_{i}(\tau)
\end{aligned}
$$

To see why (4) and (5) are sufficient to imply (3), note that

$$
\begin{aligned}
\left|\log W_{i}(\tau)-\log \log Q_{i}(\tau)\right| & \leq \max _{j \in \mathcal{N}(i)} \sqrt{\log g\left(A_{j}^{i}(\tau)\right)} \approx \max _{j \in \mathcal{N}(i)} \sqrt{\log W_{j}(\tau)} \\
& \ll \max _{\boldsymbol{\rho} \in \mathcal{I}(G)} \sum_{i^{\prime}} \rho_{i^{\prime}} \log W_{i^{\prime}}(\tau),
\end{aligned}
$$

when $\boldsymbol{W}_{\max }(\tau)$ (or $\left.\mathbf{Q}_{\max }(\tau)\right)$ is very large. Therefore,

$$
\max _{\boldsymbol{\rho} \in \mathcal{I}(G)} \sum_{i} \rho_{i} \log \log Q_{i}(\tau) \approx \max _{\boldsymbol{\rho} \in \mathcal{I}(G)} \sum_{i} \rho_{i} \log W_{i}(\tau) .
$$

In summary, to establish desired decrease in $L^{Q}(\cdot)$, it boils down to establishing (4) and (5).

To establish (4), it is essential for $g(\cdot)$ to be growing fast enough so that if $g\left(A_{j}^{i}(\tau)\right)$ is very different (in this case, smaller) compared to $W_{j}(\tau)$, then under the execution of the algorithm, it quickly converges (close) to $W_{j}(\cdot)$. For this, it is important that $g\left(A_{j}^{i}(\cdot)\right)$ should change at a faster rate compared to the rate at which $W_{j}(\cdot)$ changes. Towards that, note that if $A_{j}^{i}(\cdot)$ is updated (by unit amount) then $g\left(A_{j}^{i}(\cdot)\right)$ roughly changes by amount $g^{\prime}\left(A_{j}^{i}(\tau)\right)$, which is at least

$$
g^{\prime}\left(A_{j}^{i}(\tau)\right)>g^{\prime}\left(g^{(-1)}\left(\boldsymbol{W}_{\max }(\tau)^{3}\right)\right) .
$$

Here we have used the fact that $g^{\prime}$ is a decreasing function and $g\left(A_{j}^{i}(\tau)\right)$ is at most $\boldsymbol{W}_{\max }(\tau)^{3}$. Using properties of function $g$, we establish that (see Proposition 21) $W_{j}(\tau)$ changes per unit time by at most

$$
\frac{W_{j}(\tau)}{g^{(-1)}\left(\exp \left(\log ^{2} W_{j}(\tau)\right)\right)} .
$$


For the purpose of developing an intuition regarding the choice of $g$, consider $j \in \arg \max _{i} W_{i}(\tau)$, i.e. $W_{j}(\tau)=\boldsymbol{W}_{\max }(\tau)$. Then, such a $W_{j}(\tau)$ changes as

$$
\frac{W_{j}(\tau)}{g^{(-1)}\left(\exp \left(\log ^{2} W_{j}(\tau)\right)\right)}=\frac{\boldsymbol{W}_{\max }(\tau)}{g^{(-1)}\left(\exp \left(\log ^{2} \boldsymbol{W}_{\max }(\tau)\right)\right)} .
$$

Therefore, to have $g$ such that the change in $W_{j}(\cdot)$ is slower than that in $g\left(A_{j}^{i}(\cdot)\right)$, we must have

$$
g^{\prime}\left(g^{(-1)}\left(\boldsymbol{W}_{\max }(\tau)^{3}\right)\right)>\frac{\boldsymbol{W}_{\max }(\tau)}{g^{(-1)}\left(\exp \left(\log ^{2} \boldsymbol{W}_{\max }(\tau)\right)\right)} .
$$

Our interest will be having properties holding when $\boldsymbol{W}_{\max }(\tau)$ (or $\mathbf{Q}_{\max }(\tau)$ ) is large enough. This leads to the condition that

$$
\lim _{x \rightarrow \infty} g^{\prime}\left(g^{(-1)}\left(x^{3}\right)\right) \frac{g^{(-1)}\left(\exp \left(\log ^{2} x\right)\right)}{x}>1 .
$$

It can be checked that the above condition is satisfied if $g(x)$ does not grow slower than $\exp \left(\log \log ^{\alpha} x\right)$ for some constant $\alpha>2 .{ }^{3}$ That is, we need $g$ to be growing roughly at least as fast as the choice made in the description of our algorithm in Section 2. Precise details on how this choice of $g$ guarantees $g\left(A_{j}^{i}(\tau)\right) \approx W_{j}(\tau)$ are given in Section 7 .

Next, discussion on how we establish (5), which will require another condition on $g(\cdot)$ to be growing slow enough, in contrast to the fast enough growing condition for (4). Effectively, we need to establish that $\boldsymbol{\mu}(\tau)$, the distribution of $\boldsymbol{\sigma}(\tau)$ under the algorithm, is concentrated around the subset of schedules with high-weight, i.e. roughly speaking the subset

$$
\left\{\tilde{\boldsymbol{\rho}}=\left[\tilde{\rho}_{i}\right] \in \mathcal{I}(G): \sum_{i} \tilde{\rho}_{i} \log W_{i}(\tau) \approx \max _{\boldsymbol{\rho} \in \mathcal{I}(G)} \sum_{i} \rho_{i} \log W_{i}(\tau)\right\} .
$$

To that end, consider the evolution of schedule $\boldsymbol{\sigma}(\tau)=\left[\sigma_{i}(\tau)\right]$ and weight $\boldsymbol{W}(\tau)=\left[W_{i}(\tau)\right]$ under the algorithm. Now the distribution of $\boldsymbol{\sigma}(\tau)$ depends on the schedule $\boldsymbol{\sigma}(\tau-1)$ and weight $\boldsymbol{W}(\tau-1)$. More specifically, the evolution of $\boldsymbol{\sigma}(\tau)$ can be thought of as a time-varying Markov chain with its transition matrix $P(\tau)$ being function of the time-varying $\boldsymbol{W}(\tau)$. That is, for $\Delta \geq 1$

$$
\boldsymbol{\mu}(\tau)=\boldsymbol{\mu}(\tau-\Delta) P(\tau-\Delta) \cdots P(\tau-1) .
$$

\footnotetext{
${ }^{3}$ We say $g$ does not grow slower and faster than $f$ if $\liminf _{x \rightarrow \infty} \frac{g(x)}{f(x)}>0$ and $\limsup _{x \rightarrow \infty} \frac{g(x)}{f(x)}<\infty$, respectively.
} 
In above, we assume that the distribution $\boldsymbol{\mu}(\cdot)$ represents an $|\mathcal{I}(G)|$ dimensional row vector, $P(\cdot)$ represents an $|\mathcal{I}(G)| \times|\mathcal{I}(G)|$ probability transition matrix, and their product on the right hand side should be treated as the usual vector-matrix multiplication. The first step towards establishing concentration of $\boldsymbol{\mu}(\tau)$ around the subset of $\mathcal{I}(G)$ with high-weight (cf. (6)) is establishing the existence of an appropriate $\Delta \geq 1$ :

(a) $\Delta$ is small enough so that

$$
P(\tau-\Delta) \cdots P(\tau-1) \approx P(\tau)^{\Delta} .
$$

(b) $\Delta$ is large enough so that

$$
\boldsymbol{\mu}(\tau-\Delta) P(\tau)^{\Delta} \approx \boldsymbol{\pi}(\tau),
$$

where $\boldsymbol{\pi}(\tau)$ is the stationary distribution of $P(\tau)$, i.e. $\boldsymbol{\pi}(\tau)=\boldsymbol{\pi}(\tau) P(\tau)$.

By finding such $\Delta$, it essentially follows that $\boldsymbol{\mu}(\tau) \approx \boldsymbol{\pi}(\tau)$. The second step towards establishing concentration of $\boldsymbol{\mu}(\tau)$ around the high-weight set involves establishing that $\boldsymbol{\pi}(\tau)$ is approximately product-form with respect to the weights $\boldsymbol{W}(\tau)$ (cf. Lemma 2). Therefore, as a consequence of Gibb's maximal principle for product-form distributions, it follows that $\boldsymbol{\pi}(\tau)$ is concentrated around the subset of $\mathcal{I}(G)$ with high-weight (cf. (6)). Formally, this is stated in Proposition 17. Subsequently, this establishes that $\boldsymbol{\mu}(\tau)$ is concentrated around the subset of $\mathcal{I}(G)$ with high-weight (cf. (6))

Now we discuss the remaining task of showing the existence of $\Delta$ so that (a) and (b) are satisfied. This is where we shall discover another set of conditions on $g$ that it must be of the form $\exp \left(\log \log ^{\alpha} x\right)$ with $\alpha>2$. Now for (b) to hold, it is required that $\Delta$ is larger than the mixing time of $P(\tau)$. Using Cheeger's inequality $[4,21]$, we prove that it is sufficient to have

$$
\Delta>f_{1}\left(\boldsymbol{W}_{\max }(\tau)\right) \quad \text { with } \quad f_{1}(x)=\Theta\left(x^{6 n+1}\right) .
$$

The precise definition of $f_{1}(\cdot)$ is presented in Lemma $4 .{ }^{4}$ Next, for $\Delta$ to satisfy (a), observe that

$$
\begin{aligned}
\| P(\tau & -\Delta) \cdots P(\tau-1)-P(\tau)^{\Delta} \| \\
& \leq \sum_{s=1}^{\Delta}\left\|P(\tau-\Delta) \cdots P(\tau-s-1)(P(\tau-s)-P(\tau)) P(\tau)^{s-1}\right\| \\
& \leq \sum_{s=1}^{\Delta}\|P(\tau-s)-P(\tau)\|,
\end{aligned}
$$

\footnotetext{
${ }^{4}$ As noted in Section 4.1, we use the asymptotic notation $\Theta$ with respect to scaling in $\boldsymbol{W}_{\max }(\cdot)$ instead of $n$.
} 
where we use the triangle inequality with an appropriately defined norm $\|\cdot\|$. Further, by exploring algebraic properties of $P(\cdot)$ and $\boldsymbol{W}(\cdot)$ (cf. Proposition 16 and 21 ), we show that

$$
\|P(\tau-s)-P(\tau)\| \leq f_{2}\left(\boldsymbol{W}_{\min }(\tau)\right) \cdot s,
$$

where $\boldsymbol{W}_{\min }(\tau)=\min _{i} W_{i}(\tau)$ and $f_{2}(x)=\Theta\left(\frac{x}{g^{(-1)}\left(\exp \left(\log ^{2} x\right)\right)}\right)$. Thus, it follows that

$$
\left\|P(\tau-\Delta) \cdots P(\tau-1)-P(\tau)^{\Delta}\right\| \leq f_{2}\left(\boldsymbol{W}_{\min }(\tau)\right) \cdot \Delta^{2} .
$$

Therefore, (a) follows if $\Delta$ satisfies

$$
\Delta<\frac{\varepsilon}{\sqrt{f_{2}\left(\boldsymbol{W}_{\min }(\tau)\right)}} \quad \text { for small enough } \varepsilon>0 .
$$

From (7) and (8), it follows that a $\Delta \geq 1$ satisfying (a) and (b) exists if

$$
f_{1}\left(\boldsymbol{W}_{\max }(\tau)\right)<\frac{\varepsilon}{\sqrt{f_{2}\left(\boldsymbol{W}_{\min }(\tau)\right)}} \text { for large enough } \mathbf{Q}_{\max }(\tau) .
$$

From (1), it follows that for any $i \in V$,

$$
\begin{aligned}
W_{i}(\tau) & \geq \max _{j \in \mathcal{N}(i)} \exp \left(\sqrt{\log g\left(A_{j}^{i}(\tau)\right)}\right) \approx \max _{j \in \mathcal{N}(i)} \exp \left(\sqrt{\log W_{j}(\tau)}\right), \\
10) & \geq \exp \left(\sqrt{\log W_{j}(\tau)}\right),
\end{aligned}
$$

for any $j \in \mathcal{N}(i)$; here we have assumed $g\left(A_{j}^{i}(\tau)\right) \approx W_{j}(\tau)$. Now let $j_{*} \in$ $\arg \min _{j} W_{j}(\tau)$ and $j^{*} \in \arg \max _{j} W_{j}(\tau)$. Since $G$ is connected, there exists a path connecting $j_{*}$ and $j^{*}$ of length at most $D$ where $D \leq n-1$ is the diameter of $G$. Then by a repeated application of (10) along this path joining $j_{*}$ and $j^{*}$ starting with $j_{*}$, we obtain that

$$
\boldsymbol{W}_{\min }(\tau) \geq \exp \left(\log ^{1 / 2^{D}} \boldsymbol{W}_{\max }(\tau)\right) .
$$

Therefore, the desired inequality (9) is satisfied for large $\boldsymbol{W}_{\max }(\tau)$ if

$$
f_{1}\left(\boldsymbol{W}_{\max }(\tau)\right)<\frac{\varepsilon}{\sqrt{f_{2}\left(\exp \left(\log ^{1 / 2^{D}} \boldsymbol{W}_{\max }(\tau)\right)\right)}} .
$$

This holds if

$$
\limsup _{x \rightarrow \infty} f_{1}(x) \sqrt{f_{2}\left(\exp \left(\log ^{1 / 2^{D}} x\right)\right)}=0 .
$$

The above can be checked to hold if $g$ does not grow faster than $\exp \left(\log \log ^{\alpha} x\right)$ for some constant $\alpha<\infty$. 


\section{Preliminaries: Primary MC and Positive Recurrence.}

4.1. Notations. Let $\mathbb{Z}$ and $\mathbb{R}\left(\mathbb{Z}_{+}\right.$and $\left.\mathbb{R}_{+}\right)$denote sets of (non-negative) integers and reals, respectively. Bold letters are reserved for vector and distribution, e.g. $\mathbf{u}=\left[u_{i}\right]$ denotes a vector; $\mathbf{0}$ and $\mathbf{1}$ represent vectors of all 0's and 1's; for a function $f: \mathbb{R} \rightarrow \mathbb{R}$, we use $f(\mathbf{u})$ to denote $\left[f\left(u_{i}\right)\right]$. Similarly for a random vector $\mathbf{u}$, we use $\mathbb{E}[\mathbf{u}]$ to denote $\left[\mathbb{E}\left[u_{i}\right]\right]$. Let $\mathbf{u}_{\max }:=\max _{i} u_{i}, \mathbf{u}_{\min }:=\min _{i} u_{i}$. for a vector $\mathbf{u}$; and $\mathbf{u} \cdot \mathbf{v}$ denote the inner product $\sum_{i} u_{i} v_{i}$ of vectors $\mathbf{u}, \mathbf{v}$. We call $f: \mathbb{R} \rightarrow \mathbb{R}$ as (uniformly) $c$-Lipschitz if $|f(x)-f(y)| \leq c|x-y|$ for some constant $c>0$. Similarly, a sequence of random variables $\left\{A(\tau) \in \mathbb{R}: \tau \in \mathbb{Z}_{+}\right\}$is $c$-Lipschitz if $|A(\tau)-A(\tau+1)| \leq c$ with probability 1 , for all $\tau \in \mathbb{Z}_{+}$, for some constant $c>0$.

We will use asymptotic notations (e.g. $O, o, \Omega, \omega, \Theta$ ) with respect to scaling in queue-sizes, instead of the network size or something else. For example, we mean $n=O(1)$ and $2^{n} \mathbf{Q}_{\max }=O\left(\mathbf{Q}_{\max }\right)$ where $n$ is the number of nodes (or queues). We say function $f: \mathbb{R}_{+} \rightarrow \mathbb{R}_{+}$is polynomial by denoting $f(x)=\operatorname{poly}(x)$ if $\lim _{\sup _{x \rightarrow \infty}} \frac{f(x)}{x^{c}}=0$ for some constant $c>0$. Similarly, $f(x)=\operatorname{superpoly}(x)$ and $f(x)=\operatorname{superpolylog}(x)$ mean $\liminf _{x \rightarrow \infty} \frac{f(x)}{x^{c}}=\infty$ and $\liminf _{x \rightarrow \infty} \frac{f(x)}{\log ^{c} x}=\infty$ for any constant $c>0$, respectively.

4.2. A Markov Chain (MC) of Interest. We describe a Markov chain of finite state space, whose time-varying version will describe the evolution of the medium access algorithm described in Section 2. As we described in Section 3, our strategy for proving Theorem 1 crucially relies on understanding the stationary distribution and mixing time of the (finite state) Markov chain.

4.2.1. Description. The Markov chain evolves on state space $\mathcal{I}(G) \times$ $\{0,1\}^{n}$ and uses node weights $\boldsymbol{W}=\left[W_{i}\right] \in \mathbb{R}_{+}^{n}$ with $\boldsymbol{W}_{\min } \geq 1$. Given $(\boldsymbol{\sigma}, \boldsymbol{a}) \in \mathcal{I}(G) \times\{0,1\}^{n}$, the next (random) state $\left(\boldsymbol{\sigma}^{\prime}, \boldsymbol{a}^{\prime}\right) \in \mathcal{I}(G) \times\{0,1\}^{n}$ is obtained as follows:

1. Each node $i$ chooses $r_{i} \in\{0,1\}$ uniformly at random, i.e. $r_{i}=1$ with probability $1 / 2$ and 0 otherwise. Temporarily set

$$
a_{i}^{\prime}=\left\{\begin{array}{ll}
r_{i} & \text { if } a_{j}=0 \text { for all } j \in \mathcal{N}(i) \\
0 & \text { otherwise }
\end{array} .\right.
$$

2. Each node $i$ sets $\sigma_{i}^{\prime}$ (and possibly resets $a_{i}^{\prime}$ ) as follows: 
○ If $\sigma_{i}=1$, then set

$$
\left(\sigma_{i}^{\prime}, a_{i}^{\prime}\right)= \begin{cases}(0,0) & \text { with probability } \frac{1}{W_{i}}, \\ (1,1) & \text { otherwise }\end{cases}
$$

$\circ$ Else if $a_{j}=0$ for all $j \in \mathcal{N}(i)$, then set

$$
\sigma_{i}^{\prime}= \begin{cases}1 & \text { if } a_{i}^{\prime}=1 \text { and } a_{j}^{\prime}=0 \text { for all } j \in \mathcal{N}(i) \\ 0 & \text { otherwise }\end{cases}
$$

○ Otherwise, set $\left(\sigma_{i}^{\prime}, a_{i}^{\prime}\right)=(0,0)$.

4.2.2. Stationary distribution. Let $\Omega=\mathcal{I}(G) \times\{0,1\}^{n}$. Then $\Omega$ is the state space of the above described Markov chain; let $P_{\mathbf{x x}^{\prime}}$ denote its transition probability for $\mathbf{x}=(\boldsymbol{\sigma}, \boldsymbol{a}), \mathbf{x}^{\prime}=\left(\boldsymbol{\sigma}^{\prime}, \boldsymbol{a}^{\prime}\right) \in \Omega$. We characterize the stationary distribution of this Markov chain as follows.

Lemma 2 Starting from initial state $(\mathbf{0}, \mathbf{0})$, the Markov chain $P$ is recurrent and aperiodic; let its recurrence class be denoted by $\Omega^{\prime} \subset \Omega ;(\boldsymbol{\sigma}, \mathbf{0}) \in \Omega^{\prime}$ for all $\boldsymbol{\sigma} \in \mathcal{I}(G)$. Therefore, the Markov chain $P$ has a unique stationary distribution $\boldsymbol{\pi}$ on $\Omega^{\prime}$ such that for any $(\boldsymbol{\sigma}, \boldsymbol{a}) \in \Omega^{\prime}$

$$
\pi_{(\boldsymbol{\sigma}, \boldsymbol{a})} \propto \exp (\boldsymbol{\sigma} \cdot \log \boldsymbol{W}+U(\boldsymbol{\sigma}, \boldsymbol{a})),
$$

where $U: \Omega^{\prime} \rightarrow \mathbb{R}_{+}$is such that $|U(\boldsymbol{\sigma}, \boldsymbol{a})| \leq n 4^{n} \log 2$ for all $(\boldsymbol{\sigma}, \boldsymbol{a}) \in \Omega^{\prime}$.

To achieve the form (12), we use the classical Markov chain tree theorem [3]. Our proof strategy can be of broad interest to characterize such form for non-reversible Markov chains.

Proof. Consider the Markov chain $P$ with weights $\boldsymbol{W} \in \mathbb{R}_{+}^{n}$ such that $\boldsymbol{W}_{\min } \geq 1$. Starting from $(\mathbf{0}, \mathbf{0})$, from the description of the Markov chain, it follows inductively that Markov chain is always in state $(\boldsymbol{\sigma}, \boldsymbol{a}) \in \Omega$ so that $\boldsymbol{\sigma} \leq \boldsymbol{a}$ component-wise, i.e. for any $i, \boldsymbol{\sigma}_{i}=1 \Rightarrow a_{i}=1$. Further, transition from any such state $\mathbf{x}=(\boldsymbol{\sigma}, \boldsymbol{a}) \in \Omega$ to $\mathbf{x}^{\prime}=\left(\boldsymbol{\sigma}^{\prime}, \boldsymbol{a}^{\prime}\right) \in \Omega$ is possible if and only if (a) $\boldsymbol{\sigma} \cup \boldsymbol{\sigma}^{\prime} \in \mathcal{I}(G)$, and (b) for any $i, a_{i}=1 \Rightarrow a_{j}^{\prime}=0, \forall j \in \mathcal{N}(i)$. From (a) and (b), it immediately follows (a') $\boldsymbol{\sigma} \cup \boldsymbol{\sigma}^{\prime} \in \mathcal{I}(G)$, and (b') for any $i^{\prime}, a_{i^{\prime}}^{\prime}=1 \Rightarrow a_{j^{\prime}}=0, \forall j^{\prime} \in \mathcal{N}\left(i^{\prime}\right)$. That is, if transition from $\mathbf{x}$ to $\mathbf{x}^{\prime}$ is feasible, then transition from $\mathbf{x}^{\prime}$ to $\mathbf{x}$ is feasible. As per this, it immediately follows that the state $(\mathbf{0}, \mathbf{0})$ is reachable from (and to) all feasible states. That is, starting from $(\mathbf{0}, \mathbf{0})$ the Markov chain $P$ is recurrent and let $\Omega^{\prime}$ be the recurrent class. Further, there is a strictly positive probability of being at state $(\mathbf{0}, \mathbf{0})$. Therefore, Markov chain is aperiodic on $\Omega^{\prime}$. More generally, 
it can be checked that for any two states $\mathbf{x}, \mathbf{x}^{\prime}$ with positive $P_{\mathbf{x x}^{\prime}}$, it is equal to

$$
P_{\mathbf{x x}^{\prime}}=c\left(\mathbf{x}, \mathbf{x}^{\prime}\right) \cdot \prod_{i \in \boldsymbol{\sigma} \backslash \boldsymbol{\sigma}^{\prime}} \frac{1}{W_{i}} \cdot \prod_{i \in \boldsymbol{\sigma} \cap \boldsymbol{\sigma}^{\prime}}\left(1-\frac{1}{W_{i}}\right),
$$

where $c\left(\mathbf{x}, \mathbf{x}^{\prime}\right)=2^{-\left|\left\{i: a_{i}=0 ; a_{i}^{\prime}=1\right\}\right|}$.

Now to establish (12), consider another Markov chain $Q$ on $\Omega^{\prime}$ with $Q_{\mathbf{x x}^{\prime}}>$ 0 if and only if $P_{\mathbf{x x}^{\prime}}>0$. Specifically, for $\mathbf{x}=(\boldsymbol{\sigma}, \boldsymbol{a}), \mathbf{x}^{\prime}=\left(\boldsymbol{\sigma}^{\prime}, \boldsymbol{a}^{\prime}\right) \in \Omega^{\prime}$ with $\mathbf{x} \neq \mathbf{x}^{\prime}$ and $P_{\mathbf{x x}^{\prime}}>0$,

$$
Q_{\mathbf{x x}^{\prime}}=\frac{1}{2^{n}} \cdot \prod_{i \in \boldsymbol{\sigma} \backslash \boldsymbol{\sigma}^{\prime}} \frac{1}{W_{i}} \cdot \prod_{i \in \boldsymbol{\sigma} \cap \boldsymbol{\sigma}^{\prime}}\left(1-\frac{1}{W_{i}}\right) .
$$

We choose the other 'self-transitions' in $Q$ so as to make it a valid probability transition matrix. This is indeed possible since $Q_{\mathbf{x x}^{\prime}} \leq P_{\mathbf{x x}^{\prime}}$ for all $\mathbf{x} \neq \mathbf{x}^{\prime}$ from (13). By definition $Q$ is recurrent and aperiodic since $P$ is. Therefore, it has unique stationary distribution, say $\boldsymbol{\xi}$. We claim that for any $\mathbf{x}=$ $(\boldsymbol{\sigma}, \boldsymbol{a}) \in \Omega^{\prime}$,

$$
\boldsymbol{\xi}_{(\boldsymbol{\sigma}, \boldsymbol{a})} \propto \prod_{i \in \boldsymbol{\sigma}} W_{i}=\exp (\boldsymbol{\sigma} \cdot \log \boldsymbol{W}) \triangleq \boldsymbol{W}(\boldsymbol{\sigma}) .
$$

This is because, the following detailed-balanced condition is satisfied by $Q, \boldsymbol{\xi}$ : for any feasible transitions $\mathbf{x}=(\boldsymbol{\sigma}, \boldsymbol{a}), \mathbf{x}^{\prime}=\left(\boldsymbol{\sigma}^{\prime}, \boldsymbol{a}^{\prime}\right) \in \Omega^{\prime}$ with $\mathbf{x} \neq \mathbf{x}^{\prime}$,

$$
\frac{Q_{\mathbf{x x}^{\prime}}}{Q_{\mathbf{x}^{\prime} \mathbf{x}}}=\frac{\prod_{i \in \boldsymbol{\sigma} \backslash \boldsymbol{\sigma}^{\prime}} \frac{1}{W_{i}}}{\prod_{i \in \boldsymbol{\sigma}^{\prime} \backslash \boldsymbol{\sigma}} \frac{1}{W_{i}}}=\frac{\prod_{i \in \boldsymbol{\sigma}^{\prime} \backslash \boldsymbol{\sigma}} W_{i}}{\prod_{i \in \boldsymbol{\sigma} \backslash \boldsymbol{\sigma}^{\prime}} W_{i}}=\frac{\prod_{i \in \boldsymbol{\sigma}^{\prime}} W_{i}}{\prod_{i \in \boldsymbol{\sigma}} W_{i}}=\frac{\boldsymbol{\xi}_{\mathbf{x}^{\prime}}}{\boldsymbol{\xi}_{\mathbf{x}}} .
$$

Given characterization $\boldsymbol{\xi}$ and similarity between $Q$ and $P$, we shall approximately characterize $\boldsymbol{\pi}$, the stationary distribution of $P$, in form of $\boldsymbol{\xi}$. For this, we shall use the following proposition.

Proposition 3 Given a finite state space $\Sigma$, denoted by $\{1, \ldots, N\}$, consider two irreducible and apriodic Markov chains on $\Sigma$ with transition probability matrices $A$ and $B$. Let $A_{i j}>0$ if and only if $B_{i j}>0$ for all $i, j \in \Sigma$. Define

$$
R(A, B)=\max _{(i, j): A_{i j}>0}\left(\frac{A_{i j}}{B_{i j}}, \frac{B_{i j}}{A_{i j}}\right) .
$$

Let $\boldsymbol{\pi}^{A}$ and $\boldsymbol{\pi}^{B}$ be stationary distributions of $A$ and $B$. Then,

$$
R(A, B)^{-N} \leq \min _{i}\left(\frac{\pi_{i}^{A}}{\pi_{i}^{B}}\right) \leq \max _{i}\left(\frac{\pi_{i}^{A}}{\pi_{i}^{B}}\right) \leq R(A, B)^{N} .
$$


Subsequently, the relative entropy between $\boldsymbol{\pi}^{A}$ and $\boldsymbol{\pi}^{B}$, denoted by $D\left(\boldsymbol{\pi}^{A} \mid \boldsymbol{\pi}^{B}\right)$, is bounded above as $D\left(\boldsymbol{\pi}^{A} \mid \boldsymbol{\pi}^{B}\right) \leq N \log R(A, B)$.

Let $\boldsymbol{\pi}$ be the stationary distribution of $P$. For each $\mathbf{x} \in \Omega^{\prime}, \pi_{\mathbf{x}}>0$. Therefore, we can write

$$
\pi_{\mathbf{x}} \propto \exp (F(\mathbf{x}))
$$

with $F: \Omega^{\prime} \rightarrow \mathbb{R}$ with $F((\mathbf{0}, \mathbf{0}))=0$. Now from definition of $P$ and $Q$, it follows that

$$
R(P, Q) \leq 2^{n} .
$$

Using this, Proposition 3 and the form of $\boldsymbol{\xi}$ (cf. (15)), we have

$$
\begin{aligned}
\exp (F(\mathbf{x})) & =\frac{\pi_{\mathbf{x}}}{\pi_{(\mathbf{0}, \mathbf{0})}} \\
& =\exp (\boldsymbol{\sigma} \cdot \log W) \frac{\pi_{\mathbf{x}}}{\boldsymbol{\xi}_{\mathbf{x}}} \frac{\boldsymbol{\xi}_{(\mathbf{0}, \mathbf{0})}}{\pi_{(\mathbf{0}, \mathbf{0})}} \\
& =\exp (\boldsymbol{\sigma} \cdot \log W+U(\mathbf{x}))
\end{aligned}
$$

where $|U(\mathbf{x})| \leq\left|\Omega^{\prime}\right| \log R(P, Q) \leq n 4^{n} \log 2$. That is, we conclude that for any $\mathbf{x} \in \Omega^{\prime}$

$$
\pi_{\mathbf{x}} \propto \exp (F(\mathbf{x}))
$$

where $F(\mathbf{x})=\boldsymbol{\sigma} \cdot \log W+U(\mathbf{x})$ where $|U(\mathbf{x})| \leq n 4^{n} \log 2$. This completes the proof of Lemma 2 .

Proof. (Proposition 3) The proof follows by use of characterization of stationary distribution by means of Markov chain tree theorem (cf. [3]). Specifically, it characterizes the stationary distribution of a finite state, irreducible and aperiodic Markov chain, say $A$, as follows. Let $\mathcal{G}=(\Sigma, \mathcal{E})$ be a directed graph with $e=(i, j) \in \mathcal{E} \subset \Sigma \times \Sigma$ if $A_{i j}>0$. Then its stationary distribution, $\boldsymbol{\pi}^{A}$, is characterized as

$$
\pi_{i}^{A} \propto \sum_{T \in \mathcal{T}(i)} \prod_{(k, \ell) \in T} A_{k \ell}
$$

where $\mathcal{T}(i)$ is the set of all directed spanning trees of $\mathcal{G}$ rooted at $i$; by $(k, \ell) \in T$ we mean directed edge $(k, \ell)$ that belongs to $T$.

As per hypothesis of Proposition, it follows that the transition graph $\mathcal{G}$ for Markov chains $A$ and $B$ are identical. Therefore, from (19) it follows that 
for any $i \in \Sigma$

$$
\frac{\pi_{i}^{A}}{\pi_{i}^{B}}=\frac{\sum_{T \in \mathcal{T}(i)} \prod_{(k, \ell) \in T} A_{k \ell}}{\sum_{T \in \mathcal{T}(i)} \prod_{(k, \ell) \in T} B_{k \ell}} .
$$

By definition of $R(A, B)$, the fact that number of edges in any tree $T$ is $N=|\Sigma|$ and (20) it follows that for any $i \in \Sigma$

$$
R(A, B)^{-N} \leq \frac{\pi_{i}^{A}}{\pi_{i}^{B}} \leq R(A, B)^{N}
$$

To establish bound on relative entropy of $\boldsymbol{\pi}^{A}, \boldsymbol{\pi}^{B}$, observe that

$$
\begin{aligned}
D\left(\boldsymbol{\pi}^{A} \mid \boldsymbol{\pi}^{B}\right) & =\sum_{i} \pi_{i}^{A} \log \frac{\pi_{i}^{A}}{\pi_{i}^{B}} \\
& \leq \sum_{i} \pi_{i}^{A} \log R(A, B)^{N} \\
& =\left(\sum_{i} \pi_{i}^{A}\right) N \log R(A, B)=N \log R(A, B) .
\end{aligned}
$$

This completes the proof of Proposition 3.

4.2.3. Mixing time. Now we establish a bound on the 'mixing time' of $P$ - the time to reach near stationary distribution starting from any initial distribution. We shall use the total-variation distance: given distributions $\boldsymbol{\nu}, \boldsymbol{\mu}$ on a finite state space $\Omega^{\prime}$, define $\|\boldsymbol{\nu}-\boldsymbol{\mu}\|_{T V}=\sum_{\mathbf{x} \in \Omega^{\prime}}\left|\nu_{\mathbf{x}}-\mu_{\mathbf{x}}\right|$.

Lemma 4 Given $\varepsilon \in(0,0.5)$ with $n \geq 2$, for any distribution $\boldsymbol{\mu}$ on $\Omega^{\prime}$,

$$
\left\|\boldsymbol{\mu} P^{\tau}-\boldsymbol{\pi}\right\|_{T V}<\varepsilon
$$

for all $\tau \geq T_{\operatorname{mix}}\left(\varepsilon, n, \boldsymbol{W}_{\max }\right)$, where

$$
T_{\text {mix }} \equiv T_{\text {mix }}\left(\varepsilon, n, \boldsymbol{W}_{\max }\right)=4^{n 4^{n+1}+1} \boldsymbol{W}_{\max }^{6 n} \log \left(\frac{4^{n 4^{n}} \boldsymbol{W}_{\max }^{n}}{2 \varepsilon}\right) .
$$

Proof. By definition of $\boldsymbol{\pi}_{\min }, \boldsymbol{\pi}_{\max }$ and from (12), it follows that

$$
\begin{aligned}
\boldsymbol{\pi}_{\min } & \geq \boldsymbol{\pi}_{\max } \exp \left(-n \log \boldsymbol{W}_{\max }-n 4^{n} \log 2\right) \\
& \geq \frac{1}{\left|\Omega^{\prime}\right|} \exp \left(-n \log \boldsymbol{W}_{\max }-n 4^{n} \log 2\right) \\
& \geq \exp \left(-n \log \boldsymbol{W}_{\max }-n 4^{n} \log 4\right)=C_{n} \boldsymbol{W}_{\max }^{-n},
\end{aligned}
$$


where we have used $\left|\Omega^{\prime}\right| \leq 4^{n}, n \log 2 \geq 1$ for $n \geq 2$ and $C_{n}=\exp \left(-n 4^{n} \log 4\right)$.

Let the time-reversal of $P$ be $P^{*}$, i.e. $P_{\mathbf{x x}^{\prime}}^{*}=\pi_{\mathbf{x}^{\prime}} P_{\mathbf{x}^{\prime} \mathbf{x}} / \pi_{\mathbf{x}}$ for any $\mathbf{x}, \mathbf{x}^{\prime} \in \Omega^{\prime}$. It follows that $P P^{*}$ is a reversible Markov chain on $\Omega^{\prime}$. Then $P P^{*}$ has real eigenvalues taking values in $[-1,1]$ : let they be $-1 \leq \lambda_{\min } \leq \cdots \leq \lambda_{2} \leq \lambda_{1} \leq$ 1. It can be checked that $P P^{*}$ is irreducible and aperiodic due to structure of $P$. Therefore, it follows that $\lambda_{1}=1, \lambda_{P P^{*}}=\max \left\{\left|\lambda_{\min }\right|, \lambda_{2}\right\}<1$ and $P P^{*}$ has the unique stationary distribution equal to $\pi$, the stationary distribution of $P$, which corresponds to the (normalized) eigenvector with eigenvalue 1 . In this setting, the following is a well known [16, Corollary 1.14] bound on 'mixing time' of $P$ : starting from any initial distribution $\boldsymbol{\mu}$ on $\Omega^{\prime}$,

$$
\left\|\frac{\boldsymbol{\mu} P^{\tau}}{\boldsymbol{\pi}}-1\right\|_{2, \boldsymbol{\pi}}<\varepsilon, \text { for } \tau \geq \frac{2}{1-\lambda_{P P^{*}}} \log \frac{1}{\varepsilon \boldsymbol{\pi}_{\min }},
$$

where the $\chi^{2}$ (chi-squared) distance between two distributions on a finite state space $\left(\right.$ here $\Omega^{\prime}$ ) is defined as

$$
\left\|\frac{\boldsymbol{\nu}}{\boldsymbol{\mu}}-1\right\|_{2, \boldsymbol{\mu}}=\|\boldsymbol{\nu}-\boldsymbol{\mu}\|_{2, \frac{1}{\mu}}=\sqrt{\sum_{\mathbf{x} \in \Omega^{\prime}}\left(\frac{\nu_{\mathrm{x}}}{\mu_{\mathrm{x}}}-1\right)^{2}} .
$$

Another distance of interest is total-variation, which is defined as and related to $\chi^{2}$ distance as follows.

$$
\begin{aligned}
\frac{1}{2}\left\|\frac{\boldsymbol{\nu}}{\boldsymbol{\mu}}-1\right\|_{2, \boldsymbol{\mu}} & \geq\|\boldsymbol{\nu}-\boldsymbol{\mu}\|_{T V} \\
& =\sum_{\mathbf{x} \in \Omega^{\prime}}\left|\nu_{\mathbf{x}}-\mu_{\mathbf{x}}\right| .
\end{aligned}
$$

From (25) and (27), it follows that

$$
\left\|\boldsymbol{\mu} P^{\tau}-\boldsymbol{\pi}\right\|_{T V}<\varepsilon, \text { for } \tau \geq \frac{2}{1-\lambda_{P P^{*}}} \log \frac{1}{2 \varepsilon \boldsymbol{\pi}_{\min }} .
$$

Thus, to bound the 'mixing time' $T_{\text {mix }}$ of $P$, we need an upper bound on $\lambda_{P P^{*}}$. To that end, we shall bound the second largest eigenvalue $\lambda_{2}$ and the smallest eigenvalue $\lambda_{\min }$ in that order.

For $\lambda_{2}$, by Cheeger's inequality $[4,21]$ it is well known that

$$
\lambda_{2} \leq 1-\frac{\Phi^{2}}{2}
$$

where $\Phi$ is the conductance of $P P^{*}$, defined as

$$
\Phi=\min _{S \subset \Omega^{\prime}: \pi(S) \leq \frac{1}{2}} \frac{Q\left(S, S^{c}\right)}{\pi(S) \pi\left(S^{c}\right)},
$$


where $S^{c}=\Omega^{\prime} \backslash S, \pi(S)=\sum_{\mathbf{x} \in S} \pi_{\mathbf{x}}$ and

$$
Q\left(S, S^{c}\right)=\sum_{\mathbf{x} \in S, \mathbf{y} \in S^{c}} \pi_{\mathbf{x}}\left(P P^{*}\right)_{\mathbf{x y}} .
$$

Therefore,

$$
\begin{aligned}
\Phi & \geq \min _{S \subset \Omega} Q\left(S, S^{c}\right) \geq \min _{\left(P P^{*}\right)_{\mathbf{x}} \neq 0} \pi_{\mathbf{x}}\left(P P^{*}\right)_{\mathbf{x y}} \\
& \geq \boldsymbol{\pi}_{\min }\left(\min _{\left(P P^{*}\right)_{\mathbf{x y}} \neq 0}\left(P P^{*}\right)_{\mathbf{x y}}\right) \\
& \geq \boldsymbol{\pi}_{\min }\left(\min _{\left(P P^{*}\right)_{\mathbf{x y}} \neq 0} P_{\mathbf{x} \mathbf{0}} P_{\mathbf{y} \mathbf{0}} \frac{\pi_{\mathbf{y}}}{\pi_{\mathbf{0}}}\right), \quad \text { where } \mathbf{0}=(\mathbf{0}, \mathbf{0}) \\
& \stackrel{(a)}{\geq} C_{n} \boldsymbol{W}_{\max }^{-n} \times\left(2 \boldsymbol{W}_{\max }\right)^{-2 n} \exp \left(-n 4^{n} \ln 2\right) \\
& \geq C_{n}^{2} \boldsymbol{W}_{\max }^{-3 n},
\end{aligned}
$$

where (a) is from (24), definition of $\boldsymbol{\pi}$ from (12) and definition of $C_{n}=4^{-n 4^{n}}$. Now for $\lambda_{\min }$, we observe that for any $\mathbf{x} \in \Omega^{\prime}$,

$$
\begin{aligned}
\left(P P^{*}\right)_{\mathbf{x x}} & \geq P_{\mathbf{x} \mathbf{0}}^{2} \frac{\pi_{\mathbf{x}}}{\pi_{\mathbf{0}}} \\
& \geq\left(2 \boldsymbol{W}_{\max }\right)^{-2 n} \exp \left(-n 4^{n} \ln 2\right) \\
& \geq C_{n}^{2} \boldsymbol{W}^{-2 n} .
\end{aligned}
$$

Now it can be easily checked that $\lambda_{\min } \geq-1+2 \min _{\mathbf{x}}\left(P P^{*}\right)_{\mathbf{x x}}$. From this and (29), it follows that

$$
\lambda_{P P^{*}} \leq 1-\frac{1}{2} C_{n}^{-4} \boldsymbol{W}_{\max }^{-6 n} .
$$

Using (24),(28) and (30), it follows that starting from any initial distribution $\boldsymbol{\mu}$ on $\Omega^{\prime},\left\|\boldsymbol{\mu} P^{\tau}-\boldsymbol{\pi}\right\|_{T V}<\varepsilon$ for

$$
\tau \geq T_{\text {mix }} \triangleq 4 C_{n}^{-4} \boldsymbol{W}_{\max }^{6 n} \log \left(\frac{C_{n}^{-1} \boldsymbol{W}_{\max }^{n}}{2 \varepsilon}\right) .
$$

This completes the proof of Lemma 4 .

4.3. Ergodicity, Positive recurrence and Lyapunov-Foster. To establish optimality of the medium access algorithm, we need to show that the underlying network Markov chain, which has countably infinite state space, is ergodic, i.e. that it has the unique stationary distribution to which it converges. We briefly recall known methods from literature that will be helpful in doing so. 
Consider a discrete time Markov chain $X(\cdot)$ on countably infinite state space $X$. State $\mathrm{x} \in \mathrm{X}$ is said to be recurrent if $\mathbb{P}\left(T_{\mathrm{X}}=\infty\right)=0$, where $T_{\mathrm{x}}=\inf \{\tau \geq 1: X(\tau)=\mathrm{x}: X(0)=\mathrm{x}\}$. Specifically, a recurrent state $\mathrm{x}$ is called positive recurrent if $\mathbb{E}\left[T_{\mathrm{x}}\right]<\infty$, or else if $\mathbb{E}\left[T_{\mathrm{x}}\right]=\infty$ then it is called null recurrent. For an irreducible Markov chain, if one of its state is positive recurrent, the so are all; we call such a Markov chain positive recurrent. An irreducible, aperiodic and positive recurrent Markov chain is known to be ergodic: it has unique stationary and starting from any initial distribution, it converges (in distribution) to stationary distribution. Therefore, it is sufficient to establish positive recurrence property for establishing ergodicity of the Markov chain in addition to verifying irreducibility and aperiodicity properties. We shall recall a sufficient condition for establishing positive recurrence, known as the Lyapunov and Foster's criteria.

4.3.1. Lyapunov and Foster's criteria. This criteria utilizes existence of a "Lyapunov", "Potential" or "Energy" function of the state under evolution of the Markov chain. Specifically, consider a non-negative valued function $L: \mathrm{X} \rightarrow \mathbb{R}_{+}$such that $\sup _{\mathrm{x} \in \mathrm{X}} L(\mathrm{x})=\infty$. Let $h: \mathrm{X} \rightarrow \mathbb{Z}_{+}$be another function that is to be interpreted as a state dependent "stopping time". The 'drift' in Lyapunov function $L$ in $h$-steps starting from $\mathbf{x} \in \mathbf{X}$ is defined as

$$
\mathbb{E}[L(X(h(\mathrm{x})))-L(X(0)) \mid X(0)=\mathrm{x}] .
$$

Following is the criteria (see [5], for example):

Theorem 5 For any $\kappa>0$, let $B_{\kappa}=\{\mathrm{x}: L(\mathrm{x}) \leq \kappa\}$. Suppose there exist functions $h, k: \mathrm{X} \rightarrow \mathbb{Z}_{+}$such that for any $\mathrm{x} \in \mathrm{X}$,

$$
\mathbb{E}[L(X(h(\mathrm{x})))-L(X(0)) \mid X(0)=\mathrm{x}] \leq-k(\mathrm{x}),
$$

that satisfy the following conditions:

(L1) $\inf _{\mathrm{x} \in \mathrm{X}} k(\mathrm{x})>-\infty$.

(L2) $\liminf _{L(\mathrm{x}) \rightarrow \infty} k(\mathrm{x})>0$.

(L3) $\sup _{L(\mathrm{x}) \leq \gamma} h(\mathrm{x})<\infty$ for all $\gamma>0$.

(L4) $\lim \sup _{L(\mathrm{x}) \rightarrow \infty} h(\mathrm{x}) / k(\mathrm{x})<\infty$.

Then, there exists constant $\kappa_{0}>0$ so that for all $\kappa_{0}<\kappa$, the following holds:

$$
\begin{aligned}
\mathbb{E}\left[T_{B_{\kappa}} \mid X(0)=\mathrm{x}\right] & <\infty, \quad \text { for any } \mathrm{x} \in \mathrm{X} \\
\sup _{\mathrm{x} \in B_{\kappa}} \mathbb{E}\left[T_{B_{\kappa}} \mid X(0)=\mathrm{x}\right] & <\infty,
\end{aligned}
$$

where $T_{B_{\kappa}}:=\inf \left\{\tau \geq 1: X(\tau) \in B_{\kappa}\right\}$ i.e. the first return time to $B_{\kappa}$. In other words, $B_{\kappa}$ is positive recurrent. 
Theorem 5 implies that if $(L 1)-\left(L_{4}\right)$ are satisfied and $B_{\kappa}$ is a finite set, the Markov chain is positive recurrent.

5. Proof of Theorem 1. We shall establish ergodicity of an appropriate Markov chain describing evolution of the network under medium access algorithm as long as $\boldsymbol{\lambda} \in \boldsymbol{\Lambda}$. To that end, recall the Markov state of the network defined in the Section 3:

$$
X(\tau)=(\mathbf{Q}(\tau), \boldsymbol{\sigma}(\tau), \boldsymbol{a}(\tau), \boldsymbol{A}(\tau), \mathbf{B}(\tau)),
$$

where we recall that $\mathbf{Q}(\tau)$ represents vector of queue-sizes; $\boldsymbol{a}(\tau) \in\{0,1\}^{n}$ denotes the vector of transmission attempts by nodes at time $\tau ; \boldsymbol{\sigma}(\tau) \in \mathcal{I}(G)$ denotes the vector of resulting successful transmissions in time $\tau$ (clearly, $\boldsymbol{\sigma}(\tau) \leq \boldsymbol{a}(\tau))$; and $\boldsymbol{A}(\tau), \mathbf{B}(\tau) \in \mathbb{Z}_{+}^{2|E|}$ denoting the vector of long-term and short-term estimations maintained at nodes of the weights of their neighbors as explained in Section 2. Then it follows that under medium access algorithm $X(\cdot)$ is a Markov chain. It can be easily checked that under this Markov chain, state $\mathbf{0}$ in which all components are 0 , has positive probability of transiting to itself. Further, starting from any state, $X(\cdot)$ has positive probability of reaching state $\mathbf{0}$. Therefore, $X(\cdot)$ is always restricted to the recurrence class containing state $\mathbf{0}$; and over this class it is aperiodic. Therefore, it is sufficient to establish positive recurrence of $X(\cdot)$ over this recurrence class. Towards this, we shall utilize the following Lyapunov function $L$ and auxiliary functions $h, k$ to verify the conditions of Theorem 5 . Given state $\mathrm{x}=(\mathbf{Q}, \boldsymbol{\sigma}, \boldsymbol{a}, \boldsymbol{A}, \mathbf{B})$, define

$$
L(\mathrm{x})=\sum_{i} F\left(Q_{i}\right)+\sum_{i ; j \in \mathcal{N}(i)}\left(\left(A_{j}^{i}\right)^{2}+g^{(-1)}\left(B_{j}^{i}\right)\right),
$$

where $F(x)=\int_{0}^{x} f(y) d y$ with $f(y):=\log \log y=[\log \log y]_{+} ;$let $g^{(-1)}(x)=$ $\exp \left(\exp \left(\log ^{1 / 4} x\right)\right)$ represent the inverse function of $g(x)=\exp \left(\log \log ^{4} x\right)$. With an abuse of notation, we shall use $L(\tau)$ to denote $L(X(\tau))$.

Recall that node weights $\boldsymbol{W}$ are determined by $\mathbf{Q}$ and $\boldsymbol{A}$ as per (1). Therefore, given state $\mathrm{x}=(\mathbf{Q}, \boldsymbol{\sigma}, \boldsymbol{a}, \boldsymbol{A}, \mathbf{B})$, the weight vector $\boldsymbol{W}$ is determined. With this in mind, let

$$
C(\mathrm{x})=\max \left\{g\left(\boldsymbol{A}_{\max }\right), \mathbf{B}_{\max }\right\} .
$$

Then $h$ and $k$ are defined as

$$
\begin{aligned}
& h(\mathrm{x})=\left\{\begin{array}{cl}
C(\mathrm{x})^{n} & \text { if } C(\mathrm{x}) \geq \boldsymbol{W}_{\text {max }}^{3} \\
\frac{1}{2} \exp \left(\exp \left(\log ^{1 / 2} \boldsymbol{W}_{\max }\right)\right) & \text { otherwise. } \\
C(\mathrm{x})^{2 n} & \text { if } C(\mathrm{x}) \geq \boldsymbol{W}_{\max }^{3}
\end{array}\right. \\
& k(\mathrm{x})=\left\{\begin{array}{cl}
\log ^{1 / 2} \boldsymbol{W}_{\max } \exp \left(\exp \left(\log ^{1 / 2} \boldsymbol{W}_{\max }\right)\right) & \text { otherwise }
\end{array}\right.
\end{aligned}
$$


With these definitions, we shall establish the following.

Lemma 6 Let $\boldsymbol{\lambda} \in \boldsymbol{\Lambda}$. Then for any $\mathrm{x}$ with $L(\mathrm{x})$ large enough,

$$
\mathbb{E}[L(h(\mathrm{x}))-L(0) \mid X(0)=\mathrm{x}] \leq-k(\mathrm{x}) .
$$

It can be easily checked that $L, h$ and $k$ along with Lemma 6 satisfy conditions of Theorem 5. Now $L(\mathrm{x}) \rightarrow \infty$ as $|\mathrm{x}| \rightarrow \infty$ where $|\mathrm{x}|=|\mathbf{Q}|+|\boldsymbol{\sigma}|+$ $|\boldsymbol{a}|+|\boldsymbol{A}|+|\mathbf{B}|$ with $|\boldsymbol{\sigma}|,|\boldsymbol{a}|$ being equal to the ordering of them and $|\mathbf{Q}|,|\boldsymbol{A}|$ and $|\mathbf{B}|$ are standard 1-norm. Therefore, $B_{\kappa}=\{\mathrm{x}: L(\mathrm{x}) \leq \kappa\}$ is a finite set. Therefore, it follows that the Markov chain $X(\cdot)$ is positive recurrent; it is aperiodic and irreducible on the recurrence class containing $\mathbf{0}$ as discussed before. Therefore, it follows that it is ergodic. That is medium access algorithm of interest is optimal establishing Theorem 1. In the remainder this section, we shall establish the key Lemma 6. As explained in Section 3, the proof is divided in two cases: (a) for $\mathrm{x}$ with $C(\mathrm{x}) \geq \boldsymbol{W}_{\max }^{3}$ and (b) otherwise.

The case (a) corresponds to the situation when at least one of the estimation $g\left(A_{j}^{i}(\cdot)\right), B_{j}^{i}(\cdot)$ of $W_{j}(\cdot)$ some neighbor $j \in \mathcal{N}(i)$ for some node $i$ is quite large. Therefore, in this case, due to the nature of the algorithm, we show that there is a reduction in the Lyapunov function (part that depends on $\boldsymbol{A}(\cdot), \mathbf{B}(\cdot))$. This is argued in detail in Section 6 .

In case (b), on the other hand, all estimations are not too large. Therefore, effectively the algorithm acts as if weight of each node, say node $i$, is such that

$$
W_{i}(\cdot) \approx \max \left\{\log Q_{i}(\cdot), \max _{j \in \mathcal{N}(i)} \exp \left(\sqrt{\log W_{j}(\cdot)}\right)\right\} .
$$

Given this, as long as the $\boldsymbol{W}_{\max }$ (equivalently $\mathbf{Q}_{\max }$ ) is large enough, weight of each node is large enough (as it can be shown to be lower bounded by some increasing function of $\left.\mathbf{Q}_{\max }\right)$. Therefore, weight of each node changes very slowly : each component of $\mathbf{Q}(\cdot)$ changes at most by unit per unit time and hence if $\mathbf{Q}_{\max }$ is large then $\log \mathbf{Q}_{\max }$ changes by small amount per unit time. This essentially 'freezes' the weights over a time period that is long enough for the corresponding Markov chain of $(\boldsymbol{\sigma}(\cdot), \boldsymbol{a}(\cdot))$ to reach its stationary distribution (using bound on Mixing time cf. Lemma 4). We show that the stationary distribution has property that (with respect to it) on average the first part of the Lyapunov function decreases maximally; it results into overall negative drift if $\boldsymbol{\lambda} \in \boldsymbol{\Lambda}$. This will be useful to conclude Lemma 6 in case (b). This is argued in detail in Section 7.

6. Proof of Lemma 6: $\boldsymbol{C}(\mathbf{x}) \geq \boldsymbol{W}_{\text {max }}^{\mathbf{3}}$. The goal is to establish that starting with state $X(0)=\mathbf{x}=(\mathbf{Q}, \boldsymbol{\sigma}, \boldsymbol{a}, \boldsymbol{A}, \mathbf{B})$ such that $L(\mathrm{x})$ is large enough (to be determined in the course of the proof) and $C(\mathrm{x})=$ 
$\max \left\{g\left(\boldsymbol{A}_{\max }\right), \mathbf{B}_{\max }\right\} \geq \boldsymbol{W}_{\max }^{3}$ (with $\boldsymbol{W}$ determined based on $\mathbf{Q}, \boldsymbol{A}$ as per (1)), after time $h(\mathrm{x})=C(\mathrm{x})^{n}$ the expected value of $L$ decreases by $k(\mathrm{x})=h(\mathrm{x})^{2}=C(\mathrm{x})^{2 n}$.

$$
\mathbb{E}[L(h(\mathrm{x}))-L(0) \mid X(0)=\mathrm{x}] \leq-k(\mathrm{x}) .
$$

We note that for proving Lemma 6 , these will be the definition of functions $h$ and $k$ as it concerns the case $C(\mathrm{x}) \geq \boldsymbol{W}_{\max }^{3}$. To simplify notations, we will use notation $\mathbb{E}[\cdot]$ and $\mathbb{P}[\cdot]$ instead of $\mathbb{E}[\cdot \mid X(0)=\mathrm{x}]$ and $\mathbb{P}[\cdot \mid X(0)=\mathrm{x}]$ whenever it is clear from the context.

To that end, note that if $L(\mathrm{x})$ is large enough, then either $\mathbf{Q}_{\max }, \boldsymbol{A}_{\max }$ or $\mathbf{B}_{\max }$ is large. Now if $\mathbf{Q}_{\max }$ or $\boldsymbol{A}_{\max }$ are large then necessarily $\boldsymbol{W}_{\max }$ is large. Since $C(\mathrm{x})$ depends on $\boldsymbol{A}_{\max }, \mathbf{B}_{\max }$ and since we have $C(\mathrm{x}) \geq \boldsymbol{W}_{\max }^{3}$, it necessarily follows that $C(\mathrm{x})$ is large due to $L(\mathrm{x})$ being large. Now for large enough $L(\mathrm{x})$ and hence large enough $C(\mathrm{x})$,

$$
h(\mathrm{x})=C(\mathrm{x})^{n} \leq \frac{g^{(-1)}\left(\exp \left(\log ^{2}(\sqrt{C(\mathrm{x}) / 2}-1)\right)\right)}{2(\sqrt{C(\mathrm{x}) / 2}-1)} .
$$

The above holds for large enough $C(\mathrm{x})$ because $g^{(-1)}\left(\exp \left(\log ^{2} x\right)\right)$ is a superpolynomial function of $x$, i.e.

$$
\frac{g^{(-1)}\left(\exp \left(\log ^{2} x\right)\right)}{x^{c}} \rightarrow \infty, \quad \text { as } x \rightarrow \infty, \quad \text { for any fixed } c>0 .
$$

Therefore, from (90) of Corollary 22 (presented in Appendix), it follows that for all $\tau \leq h(\mathrm{x})$,

$$
\boldsymbol{W}_{\max }(\tau) \leq \sqrt{C(\mathrm{x}) / 2}:=\mathcal{W}_{\max } .
$$

Two Lemmas. Now we state two key lemmas that will lead to (36). We shall present their proofs in Section 6.1 and 6.2 respectively.

Lemma 7 Given initial state $X(0)=\mathrm{x}=(\mathbf{Q}, \boldsymbol{\sigma}, \boldsymbol{a}, \boldsymbol{A}, \mathbf{B})$, let $C(\mathrm{x}) \geq \boldsymbol{W}_{\max }^{3}$ and $C(\mathrm{x})$ be large enough. Then for any $i$ and $j \in \mathcal{N}(i)$

$$
\mathbb{E}\left[A_{j}^{i}(h(\mathrm{x}))^{2}\right] \leq \begin{cases}\left(A_{j}^{i}\right)^{2}-\frac{A_{j}^{i} h(\mathrm{x})}{O\left(g\left(A_{j}^{i}\right)^{\frac{n+1}{2}}\right)}+O\left(A_{j}^{i}\right) & \text { if } g\left(A_{j}^{i}\right)>\frac{C(\mathrm{x})}{2}, \\ \left(A_{j}^{i}+h(\mathrm{x})\right)^{2} & \text { otherwise. }\end{cases}
$$

Lemma 8 Given initial state $X(0)=\mathrm{x}=(\mathbf{Q}, \boldsymbol{\sigma}, \boldsymbol{a}, \boldsymbol{A}, \mathbf{B})$, let $C(\mathrm{x}) \geq \boldsymbol{W}_{\max }^{3}$ and $C(\mathrm{x})$ be large enough. Then for any $i$ and $j \in \mathcal{N}(i)$

$$
\mathbb{E}\left[g^{(-1)}\left(B_{j}^{i}(h(\mathrm{x}))\right)\right] \leq O\left(g^{(-1)}(C(\mathrm{x}) / 2)\right) .
$$


Implications of Lemmas 7 and 8. Define five different events as follows:

$$
\begin{aligned}
& S_{1}=\left\{(i, j) \in E: g\left(A_{j}^{i}\right)=C(\mathrm{x})\right\} \\
& S_{2}=\left\{(i, j) \in E: C(\mathrm{x}) / 2<g\left(A_{j}^{i}\right)<C(\mathrm{x})\right\} \\
& S_{3}=\left\{(i, j) \in E: g\left(A_{j}^{i}\right) \leq C(\mathrm{x}) / 2\right\} \\
& S_{4}=\left\{(i, j) \in E: B_{j}^{i}=C(\mathrm{x})\right\} \\
& S_{5}=\left\{(i, j) \in E: B_{j}^{i}<C(\mathrm{x})\right\} .
\end{aligned}
$$

For $(i, j) \in S_{1}$, using relation $g\left(A_{j}^{i}\right)=C(\mathrm{x}), h(\mathrm{x})=C(\mathrm{x})^{n}$ and Lemma 7 , we have

$$
\begin{aligned}
\mathbb{E}\left[A_{j}^{i}(h(\mathrm{x}))^{2}-\left(A_{j}^{i}\right)^{2}\right] & \leq-\frac{A_{j}^{i} h(\mathrm{x})}{O\left(g\left(A_{j}^{i}\right)^{\frac{n+1}{2}}\right)}+O\left(A_{j}^{i}\right) \\
& =-\frac{g^{(-1)}(C(\mathrm{x})) C(\mathrm{x})^{n}}{O\left(C(\mathrm{x})^{\frac{n+1}{2}}\right)}+O\left(g^{(-1)}(C(\mathrm{x}))\right) \\
& \leq-\frac{1}{2} g^{(-1)}(C(\mathrm{x})),
\end{aligned}
$$

where the last inequality follows for large enough $C(\mathrm{x})$.

For $(i, j) \in S_{2}$, it follows from Lemma 7 that for large enough value of $C(\mathrm{x})$

$$
\mathbb{E}\left[A_{j}^{i}(h(\mathrm{x}))^{2}-\left(A_{j}^{i}\right)^{2}\right] \leq 0,
$$

where we use $g\left(A_{j}^{i}\right)^{\frac{n+1}{2}}=o\left(C(\mathrm{x})^{n}\right)$ and $g\left(A_{j}^{i}\right)=\Omega(C(\mathrm{x}))$.

For $(i, j) \in S_{3}$, Lemma 7 implies that

$$
\begin{gathered}
\mathbb{E}\left[A_{j}^{i}(h(\mathrm{x}))^{2}-\left(A_{j}^{i}\right)^{2}\right] \leq\left(A_{j}^{i}+h(\mathrm{x})\right)^{2}-\left(A_{j}^{i}\right)^{2}=2 A_{j}^{i} h(\mathrm{x})+h(\mathrm{x})^{2} \\
\leq 2 g^{(-1)}\left(\frac{C(\mathrm{x})}{2}\right) h(\mathrm{x})+h(\mathrm{x})^{2}=O\left(g^{(-1)}\left(\frac{C(\mathrm{x})}{2}\right)\right) C(\mathrm{x})^{n},
\end{gathered}
$$

where the last inequality utilizes the super-polynomial property of $g^{(-1)}(\cdot)$ function:

$$
h(\mathrm{x})=C(\mathrm{x})^{n}=o\left(g^{(-1)}\left(\frac{C(\mathrm{x})}{2}\right)\right) .
$$

For $(i, j) \in S_{4}$, Lemma 8 implies that

$$
\begin{aligned}
\mathbb{E}\left[g^{(-1)}\left(B_{j}^{i}(h(\mathrm{x}))\right)-g^{(-1)}\left(B_{j}^{i}\right)\right] & \leq O\left(g^{(-1)}\left(\frac{C(\mathrm{x})}{2}\right)\right)-g^{(-1)}(C(\mathrm{x})) \\
& \leq-\frac{1}{2} g^{(-1)}(C(\mathrm{x})),
\end{aligned}
$$


where the last equality follows for $C(\mathrm{x})$ large enough from the following proposition stating the super-polynomial property of $g^{(-1)}(\cdot)$ function; we skip the proof as it is elementary.

Proposition 9 For any given $k \in \mathbb{Z}_{+}$,

$$
\lim _{x \rightarrow \infty} \frac{x^{k} g^{(-1)}(x / 2)}{g^{(-1)}(x)}=0 .
$$

For $(i, j) \in S_{5}$, Lemma 8 implies that

$$
\mathbb{E}\left[g^{(-1)}\left(B_{j}^{i}(h(\mathrm{x}))\right)\right]=O\left(g^{(-1)}\left(\frac{C(\mathrm{x})}{2}\right)\right) .
$$

From (38), (39), (40), (42) and (44), it follows that

$$
\begin{aligned}
& \mathbb{E}\left[\sum_{i, j} A_{j}^{i}(h(\mathrm{x}))^{2}+\sum_{i, j} g^{(-1)}\left(B_{j}^{i}(h(\mathrm{x}))\right)\right]-\sum_{i, j}\left(A_{j}^{i}\right)^{2}-\sum_{i, j} g^{(-1)}\left(B_{j}^{i}\right) \\
& \quad \leq-\left(\frac{\left|S_{1}\right|+\left|S_{4}\right|}{2}\right) g^{(-1)}(C(\mathrm{x}))+\left(\left|S_{3}\right|+\left|S_{5}\right|\right) O\left(g^{(-1)}\left(\frac{C(\mathrm{x})}{2}\right)\right) C(\mathrm{x})^{n} \\
& \quad \stackrel{(a)}{=}-\frac{1}{2} g^{(-1)}(C(\mathrm{x}))+O\left(n^{2} g^{(-1)}\left(\frac{C(\mathrm{x})}{2}\right)\right) C(\mathrm{x})^{n} \\
& \quad \stackrel{(b)}{\leq}-\frac{1}{4} g^{(-1)}(C(\mathrm{x})),
\end{aligned}
$$

where (a) is from $\left|S_{1}\right|+\left|S_{4}\right| \geq 1$ and $\left|S_{3}\right|+\left|S_{5}\right| \leq|E| \leq n^{2}$; (b) is from Proposition 9. 
Concluding (36). From the above inequality, we obtain the following:

$$
\begin{aligned}
\mathbb{E}[L(h(\mathrm{x}))-L(0)] \\
=\mathbb{E}\left[\sum_{i} F\left(Q_{i}(h(\mathrm{x}))\right)+\sum_{i, j} A_{j}^{i}(h(\mathrm{x}))^{2}+\sum_{i, j} g^{(-1)}\left(B_{j}^{i}(h(\mathrm{x}))\right)\right] \\
\quad-\mathbb{E}\left[\sum_{i} F\left(Q_{i}\right)+\sum_{i, j}\left(A_{j}^{i}\right)^{2}+\sum_{i, j} g^{(-1)}\left(B_{j}^{i}\right)\right] \\
\leq \mathbb{E}\left[\sum_{i} F\left(Q_{i}(h(\mathrm{x}))\right)-F\left(Q_{i}\right)\right]-\frac{1}{4} g^{(-1)}(C(\mathrm{x})) \\
\quad \stackrel{(c)}{\leq} \sum_{i}\left(F\left(Q_{i}+h(\mathrm{x})\right)-F\left(Q_{i}\right)\right)-\frac{1}{4} g^{(-1)}(C(\mathrm{x})) \\
\leq \sum_{i} f\left(Q_{i}+h(\mathrm{x})\right) h(\mathrm{x})-\frac{1}{4} g^{(-1)}(C(\mathrm{x})) \\
\leq n f\left(\mathbf{Q}_{\max }+h(\mathrm{x})\right) h(\mathrm{x})-\frac{1}{4} g^{(-1)}(C(\mathrm{x})) \\
\leq n f\left(\exp \left(\boldsymbol{W}_{\max }\right)+h(\mathrm{x})\right) h(\mathrm{x})-\frac{1}{4} g^{(-1)}(C(\mathrm{x})) \\
\quad \stackrel{(d)}{\leq} n f\left(\exp \left(C(\mathrm{x})^{1 / 3}\right)+C(\mathrm{x})^{n}\right) C(\mathrm{x})^{n}-\frac{1}{4} g^{(-1)}(C(\mathrm{x})) \\
\quad \stackrel{(e)}{=}-\frac{1}{8} g^{(-1)}(C(\mathrm{x})) \stackrel{(f)}{\leq}-C(\mathrm{x})^{2 n},
\end{aligned}
$$

where (c) is from 1-Lipschitz property of $Q_{i}(\cdot)$; (d) is from $C(\mathrm{x}) \geq \boldsymbol{W}_{\max }^{3}$; (e) and (f) hold for large enough $C(\mathrm{x})$ due to the fact that $f(x)=\log \log x$ and $g^{(-1)}(x)$ has the super-polynomial growth property as per Proposition 9. This completes the proof of Lemma 6 for the case $C(\mathrm{x}) \geq \boldsymbol{W}_{\max }^{3}$.

6.1. Proof of Lemma 7. Observe that Lemma 7 for the case $g\left(A_{j}^{i}\right) \leq$ $C(\mathrm{x}) / 2$ follows immediately from the 1-Lipschitz property of $A_{j}^{i}(\cdot)$. Hence, we shall only consider the case when $g\left(A_{j}^{i}\right)>C(\mathrm{x}) / 2$.

Define $\mathcal{W}_{\max }=\sqrt{C(\mathrm{x}) / 2}$. Then from $(37)$

$$
\boldsymbol{W}_{\max }(\tau)<\mathcal{W}_{\max }<\sqrt{g\left(A_{j}^{i}\right)}, \quad \text { for all } \tau \leq h(\mathrm{x}) .
$$

For the purpose of the proof, consider modification of the original network Markov chain, say original Markov chain be $M$ and let its modification be $M^{\prime}$. Under this modification, the $M^{\prime}$ evolves in the same manner as $M$ for $\tau \leq h(\mathrm{x})$; for $\tau>h(\mathrm{x})$ the evolution of $M^{\prime}$ is the same as that of $M$ 
except $\boldsymbol{W}(\tau)=\boldsymbol{W}(\tau-1)$ i.e. $\boldsymbol{W}(\cdot)$ remains fixed to its value at time $h(\mathrm{x})$. Clearly, the quantity of interest $\mathbb{E}\left[A_{j}^{i}(h(\mathrm{x}))^{2}\right]$ in Lemma 7 remains invariant with respect to $M$ and $M^{\prime}$. As mentioned earlier, this modification is for convenience of proof and it merely guarantees (45) for all $\tau$. Therefore, we shall bound $\mathbb{E}\left[A_{j}^{i}(h(\mathrm{x}))^{2}\right]$ under $M^{\prime}$ for which we have

$$
\boldsymbol{W}_{\max }(\tau)<\mathcal{W}_{\max }<\sqrt{g\left(A_{j}^{i}\right)}, \quad \text { for all } \tau \geq 0 .
$$

With respect to $M^{\prime}$, define random times $0=T_{0}<T_{1}<T_{2} \ldots$ such that $T_{m}$ is the $m$ th time when $A_{j}^{i}(\cdot)$ is updated, i.e. $B_{j}^{i}\left(T_{m}-1\right) \geq 2$ and $B_{j}^{i}\left(T_{m}\right)=0$. Define for $m \geq 0$,

$$
Y_{m}= \begin{cases}A_{j}^{i}\left(T_{m}\right)^{2} & \text { if } T_{m-1} \leq h(\mathrm{x}) \text { or } m=0 \\ Y_{m-1}-A_{j}^{i} & \text { otherwise. }\end{cases}
$$

Let $m^{*}=\inf \left\{m \geq 0: T_{m}>h(\mathrm{x})\right\}$. Then it follows that

$$
A_{j}^{i}(h(\mathrm{x}))^{2}=A_{j}^{i}\left(T_{m^{*}-1}\right)^{2}=Y_{m^{*}-1} .
$$

We establish the following property of $Y_{m}$.

Proposition 10 Given $g\left(A_{j}^{i}\right)>C(\mathrm{x}) / 2$, for $m \geq 1$

$$
\mathbb{E}\left[Y_{m+1} \mid \mathcal{F}_{m}\right] \leq Y_{m}-A_{j}^{i},
$$

where $\mathcal{F}_{m}$ denotes the filtration containing $Y_{k}, T_{k}$ for $0 \leq k \leq m$.

Proof. If $T_{m}>h(\mathrm{x})$, then the desired result follows from definition of $Y_{m}$. Now suppose $T_{m} \leq h(\mathrm{x})$. Then observe that

$$
\begin{aligned}
g\left(A_{j}^{i}\left(T_{m}\right)\right) & \stackrel{(a)}{\geq} g\left(A_{j}^{i}-h(\mathrm{x})\right) \\
& \stackrel{(b)}{\geq} g\left(A_{j}^{i}\right)-h(\mathrm{x}) g^{\prime}(c), \quad \text { for some } c \in\left(A_{j}^{i}-h(\mathrm{x}), A_{j}^{i}\right) \\
& \geq g\left(A_{j}^{i}\right)-h(\mathrm{x}) g^{\prime}\left(A_{j}^{i}-h(\mathrm{x})\right) \\
& \stackrel{(c)}{\geq} g\left(A_{j}^{i}\right)-1,
\end{aligned}
$$

where (a) is from 1-Lipschitz property of $A_{j}^{i}(\cdot)$; (b) is from the mean value theorem; for (c) we use the following that holds for large enough $C(\mathrm{x})$ and $g\left(A_{j}^{i}\right) \geq C(\mathrm{x}) / 2$ (along with the definition of $g(\cdot)$ )

$$
h(\mathrm{x})=C(\mathrm{x})^{n} \leq 2^{-n} g\left(A_{j}^{i}\right)^{n}<\sqrt{A_{j}^{i}}
$$


and $g^{\prime}(x)<1 / \sqrt{x}$ for large enough $x$.

Now we bound the probability that $A_{j}^{i}$ increases at time $T_{m+1}$ :

$$
\begin{aligned}
\mathbb{P}\left(A_{j}^{i}\left(T_{m+1}\right)=A_{j}^{i}\left(T_{m}\right)+1 \mid \mathcal{F}_{m}\right) & =\mathbb{P}\left(B_{j}^{i}\left(T_{m+1}-1\right) \geq g\left(A_{j}^{i}\left(T_{m}\right)\right) \mid \mathcal{F}_{m}\right) \\
& \stackrel{(a)}{\leq} \mathbb{P}\left(B_{j}^{i}\left(T_{m+1}-1\right) \geq g\left(A_{j}^{i}\right)-1 \mid \mathcal{F}_{m}\right) \\
& \stackrel{(b)}{<}\left(1-\frac{1}{\mathcal{W}_{\max }}\right)^{g\left(A_{j}^{i}\right)-2} \\
& \stackrel{(c)}{<}\left(1-\frac{1}{\sqrt{g\left(A_{j}^{i}\right)}}\right)^{g\left(A_{j}^{i}\right)-2} \\
(50) \quad & \stackrel{(d)}{\leq} \frac{1}{10} .
\end{aligned}
$$

In above, (a) and (c) are from (49) and (46) when assuming $A_{j}^{i}$ (equivalently $C(\mathrm{x})$ ) is large enough; for (b) we observe that $\boldsymbol{W}_{\max }(\tau)$ is uniformly bounded above by $\mathcal{W}_{\max }$ from (46); (d) is again due to large enough $A_{j}^{i}$. Therefore, once $j$ is successful in its transmission, the probability that $j$ consecutively attempts to transmit (without stopping) for an interval of length $k$ is at $\operatorname{most}\left(1-\frac{1}{\mathcal{W}_{\max }}\right)^{k}$. Using (50), it follows that

$$
\begin{aligned}
\mathbb{E}\left[Y_{m+1} \mid \mathcal{F}_{m}\right] & =\mathbb{E}\left[A_{j}^{i}\left(T_{m+1}\right)^{2} \mid \mathcal{F}_{m}\right] \\
& \leq \frac{1}{10}\left(A_{j}^{i}\left(T_{m}\right)+1\right)^{2}+\frac{9}{10}\left(A_{j}^{i}\left(T_{m}\right)-1\right)^{2} \\
& =A_{j}^{i}\left(T_{m}\right)^{2}-\frac{8}{5} A_{j}^{i}\left(T_{m}\right) \\
& \leq A_{j}^{i}\left(T_{m}\right)^{2}-\frac{8}{5} A_{j}^{i}+\frac{8}{5} T_{m} \\
& \leq A_{j}^{i}\left(T_{m}\right)^{2}-\frac{8}{5} A_{j}^{i}+\frac{8}{5} h(\mathrm{x}) \\
& \leq Y_{m}-A_{j}^{i},
\end{aligned}
$$

where we used 1-Lipschitz property of $A_{j}^{i}(\cdot), T_{m} \leq h(\mathrm{x})$ and the fact that $h(\mathrm{x})=C(\mathrm{x})^{n} \leq 2^{-n} g\left(A_{j}^{i}\right)^{n}=o\left(A_{j}^{i}\right)$. This completes the proof of Proposition 10.

Completing proof of Lemma \%. Define $Z_{m}=Y_{m}+(m-1) A_{j}^{i}$. Then as per Proposition 10, $\left\{Z_{m}: m \geq 1\right\}$ is a sub-martingale with respect to $\mathcal{F}_{m}$. By the Doob's optional stopping theorem, we have that

$$
\mathbb{E}\left[Z_{m^{*}}\right] \leq \mathbb{E}\left[Z_{1}\right]=\mathbb{E}\left[Y_{1}\right] .
$$


Therefore, the desired inequality follows as

$$
\begin{aligned}
\mathbb{E}\left[A_{j}^{i}(h(\mathrm{x}))^{2}\right] & =\mathbb{E}\left[A_{j}^{i}\left(T_{m^{*}-1}\right)^{2}\right] \stackrel{(a)}{\leq} \mathbb{E}\left[\left(A_{j}^{i}\left(T_{m^{*}}\right)+1\right)^{2}\right] \\
& =\mathbb{E}\left[A_{j}^{i}\left(T_{m^{*}}\right)^{2}\right]+2 \mathbb{E}\left[A_{j}^{i}\left(T_{m^{*}}\right)\right]+1 \\
& \stackrel{(b)}{\leq} \mathbb{E}\left[Y_{m^{*}}\right]+2 \mathbb{E}\left[A_{j}^{i}+m^{*}\right]+1 \\
& =\mathbb{E}\left[Z_{m^{*}}-\left(m^{*}-1\right) A_{j}^{i}\right]+2 \mathbb{E}\left[A_{j}^{i}+m^{*}\right]+1 \\
& \leq \mathbb{E}\left[Y_{1}\right]-\mathbb{E}\left[m^{*}\right]\left(A_{j}^{i}-2\right)+3 A_{j}^{i}+1 \\
& =\mathbb{E}\left[A_{j}^{i}\left(T_{1}\right)^{2}\right]-\mathbb{E}\left[m^{*}\right]\left(A_{j}^{i}-2\right)+3 A_{j}^{i}+1 \\
& \stackrel{(c)}{\leq}\left(A_{j}^{i}+1\right)^{2}-\mathbb{E}\left[m^{*}\right]\left(A_{j}^{i}-2\right)+3 A_{j}^{i}+1 \\
& =\left(A_{j}^{i}\right)^{2}-\mathbb{E}\left[m^{*}\right]\left(A_{j}^{i}-2\right)+5 A_{j}^{i}+2 \\
& \stackrel{(d)}{=}\left(A_{j}^{i}\right)^{2}-\frac{h(\mathrm{x})}{O\left(g\left(A_{j}^{i}\right)^{\frac{n+1}{2}}\right)} A_{j}^{i}+O\left(A_{j}^{i}\right),
\end{aligned}
$$

where (a), (b), (c) are from the 1-Lipschitz property of $A_{j}^{i}(\cdot)$ and (d) is due to the following proposition. This completes the proof of Lemma 7.

Proposition 11 For large enough $C(\mathrm{x})$,

$$
\mathbb{E}\left[m^{*}\right] \geq \frac{h(\mathrm{x})}{O\left(g\left(A_{j}^{i}\right)^{\frac{n+1}{2}}\right)}+1 .
$$

Proof. For $1 \leq \tau \leq h(\mathrm{x})$, define

$$
U_{\tau}= \begin{cases}1 & \text { if } A_{j}^{i}(\cdot) \text { is updated at time } \tau \\ 0 & \text { otherwise }\end{cases}
$$

That is, $U_{\tau}=1$ iff $B_{j}^{i}(\tau-1) \geq 2$ and $B_{j}^{i}(\tau)=0$. By definition of $U_{\tau}$ and $m^{*}$,

$$
m^{*}-1=\sum_{\tau=1}^{h(\mathrm{x})} U_{\tau} .
$$

Therefore, to bound $\mathbb{E}\left[m^{*}\right]$ we next bound $\mathbb{E}\left[U_{\tau}\right]$. For any $5 \leq \tau \leq h(\mathrm{x})-5$, let $X(\tau-5)=\{\mathbf{Q}(\tau-5), \boldsymbol{\sigma}(\tau-5), \boldsymbol{a}(\tau-5), \boldsymbol{A}(\tau-5), \mathbf{B}(\tau-5)\}$ be the 
network state at time $\tau-5$. For this, define event $\mathfrak{E}$ :

$$
\begin{aligned}
\mathfrak{E} & =\mathfrak{E}_{1}^{\prime} \& \mathfrak{E}_{2}^{\prime} \& \mathfrak{E}_{3}^{\prime} \\
\mathfrak{E}_{1} & =\text { all nodes do not attempt to transmit at time } \tau-4 \\
\mathfrak{E}_{2} & =\text { Only } j \text { attemtps to trasmit at time } \tau-2 \text { and } \tau-3 \\
\mathfrak{E}_{3} & =j \text { does not attempt to transmit at time } \tau-1 .
\end{aligned}
$$

If $\mathfrak{E}$ happens, $A_{j}^{i}$ is updated at time $\tau$ i.e. $U_{\tau}=1$. First note that

$$
\mathbb{P}\left[\mathfrak{E}_{1}\right] \geq\left(\frac{1}{\mathcal{W}_{\max }}\right)^{n}=\Omega\left(\frac{1}{g\left(A_{j}^{i}\right)^{n / 2}}\right),
$$

whether this naive lower bound is obtained from (46) and the case when many nodes (as possible) succeed in their transmissions at time $\tau-4$. Second we have

$$
\mathbb{P}\left[\mathfrak{E}_{2} \mid \mathfrak{E}_{1}\right] \geq\left(\frac{1}{2}\right)^{n} \times\left(\frac{1}{2}\right)^{n}=\Omega(1)
$$

The above lower bound is obtained considering the scenario that the balanced coin of $j$ produces 'head' at times $\tau-2$ and $\tau-3$; coins of all other nodes produce 'tail' at times $\tau-2$ and $\tau-3$. Third since the transmission of $j$ is successful at time $\tau-2$, it is easy to see that

$$
\mathbb{P}\left[\mathfrak{E}_{3} \mid \mathfrak{E}_{2}\right] \geq \frac{1}{\mathcal{W}_{\max }}=\Omega\left(1 / \sqrt{g\left(A_{j}^{i}\right)}\right),
$$

from (46). By combining (51), (52) and (53),

$$
\begin{aligned}
\mathbb{P}\left[U_{\tau}\right. & =1 \mid X(\tau-5)] \\
& \geq \mathbb{P}[\mathfrak{E} \mid X(\tau-5)] \\
& =\mathbb{P}\left[\mathfrak{E}_{1} \& \mathfrak{E}_{2} \& \mathfrak{E}_{3} \mid X(\tau-5)\right] \\
& =\Omega\left(g\left(A_{j}^{i}\right)^{-\frac{n+1}{2}}\right) .
\end{aligned}
$$

The above inequality holds for any given $X(\tau-5)$. Hence,

$$
\mathbb{P}\left[U_{\tau}=1\right]=\Omega\left(g\left(A_{j}^{i}\right)^{-\frac{n+1}{2}}\right) .
$$


Finally, the conclusion follows as

$$
\begin{aligned}
\mathbb{E}\left[m^{*}-1\right] & \geq \mathbb{E}\left[\sum_{\tau=5}^{h(\mathrm{x})} U_{\tau}\right] \\
& =\sum_{\tau=5}^{h(\mathrm{x})} \mathbb{E}\left[U_{\tau}\right] \\
& =\sum_{\tau=5}^{h(\mathrm{x})} \mathbb{P}\left[U_{\tau}=1\right] \\
& =(h(\mathrm{x})-4) \cdot \Omega\left(g\left(A_{j}^{i}\right)^{-\frac{n+1}{2}}\right) \\
& =\frac{h(\mathrm{x})}{O\left(g\left(A_{j}^{i}\right)^{\frac{n+1}{2}}\right)} .
\end{aligned}
$$

6.2. Proof of Lemma 8. Let $\tau^{*}=\inf \left\{\tau \geq 1: a_{j}(\tau)=0\right\}$ i.e. the first time $j$ does not attempt to transmit, and let the event $\mathfrak{E}$ denote $\tau^{*} \geq h(\mathrm{x})$. Hence, if $\mathfrak{E}$ happens, $B_{j}^{i}(h(\mathrm{x}))=B_{j}^{i}+h(\mathrm{x})$ and transmissions of $j$ should be successful consecutively for time $\tau \in[0, h(\mathrm{x})-2]$ (otherwise, $j$ would have stopped attempting). Under this observation, we obtain

$\mathbb{P}[\mathfrak{E}] \leq \mathbb{P}[j$ attempts to transmit consecutively for time $\tau \in[1, h(\mathrm{x})-1]]$ $(54) \leq\left(1-\frac{1}{\mathcal{W}_{\max }}\right)^{h(\mathrm{x})-1}$,

where the last inequality follows from the fact that $W_{j}(\tau)$ is bounded from above by $\mathcal{W}_{\text {max }}$ as per (37). On the other hand, if the event $\mathfrak{E}$ does not happen, $j$ stops attempting transmission before time $h(\mathrm{x})$. Hence $B_{j}^{i}$ should set to 0 before time $h(\mathrm{x})$. Based on this observation and arguments similar to those used for establishing (54), we obtain

$$
\begin{aligned}
\mathbb{P}\left[B_{j}^{i}(h(\mathrm{x}))=k \mid \mathfrak{E}^{c}\right] \leq \mathbb{P}[j \text { attempts to transmit consecutively } \\
\quad \text { for time } \tau \in[h(\mathrm{x})-k+1, h(\mathrm{x})-1]] \\
\leq\left(1-\frac{1}{\mathcal{W}_{\max }}\right)^{k-1} .
\end{aligned}
$$

Now observe that

$\mathbb{E}\left[g^{(-1)}\left(B_{j}^{i}(h(\mathrm{x}))\right)\right]=\mathbb{P}[\mathfrak{E}] \mathbb{E}\left[g^{(-1)}\left(B_{j}^{i}(h(\mathrm{x}))\right) \mid \mathfrak{E}\right]+\mathbb{P}\left[\mathfrak{E}^{c}\right] \mathbb{E}\left[g^{(-1)}\left(B_{j}^{i}(h(\mathrm{x}))\right) \mid \mathfrak{E}^{c}\right]$

(56) $\quad \leq \mathbb{P}[\mathfrak{E}] \mathbb{E}\left[g^{(-1)}\left(B_{j}^{i}(h(\mathrm{x}))\right) \mid \mathfrak{E}\right]+\mathbb{E}\left[g^{(-1)}\left(B_{j}^{i}(h(\mathrm{x}))\right) \mid \mathfrak{E}^{c}\right]$. 
For the first term in (56), we consider the following using (54):

$$
\begin{aligned}
\mathbb{P}[\mathfrak{E}] \mathbb{E}\left[g^{(-1)}\left(B_{j}^{i}(h(\mathrm{x}))\right) \mid \mathfrak{E}\right] & \leq\left(1-\frac{1}{\mathcal{W}_{\max }}\right)^{h(\mathrm{x})-1} \cdot g^{(-1)}\left(B_{j}^{i}+h(\mathrm{x})\right) \\
& \leq\left(1-\frac{1}{\sqrt{C(\mathrm{x}) / 2}}\right)^{C(\mathrm{x})^{n}-1} \cdot g^{(-1)}\left(C(\mathrm{x})+C^{n}(\mathrm{x})\right) \\
& =O(1),
\end{aligned}
$$

where the last inequality follows for $C(\mathrm{x})$ large enough, i.e. $\boldsymbol{W}_{\max }$ large enough. In above we have used the definition $h(\mathrm{x})=C(\mathrm{x})^{n}$ with $C(\mathrm{x})=$ $\max \left\{g\left(\boldsymbol{A}_{\max }\right), B_{\max }\right\}$ which is at least $\boldsymbol{W}_{\max }^{3}$. For the second term in (56), we consider the following using (55):

$$
\begin{aligned}
\mathbb{E}\left[g^{(-1)}\left(B_{j}^{i}(h(\mathrm{x}))\right) \mid \mathfrak{E}^{c}\right] & \leq \sum_{k=1}^{\infty} g^{(-1)}(k) \cdot\left(1-\frac{1}{\mathcal{W}_{\max }}\right)^{k-1} \\
& \stackrel{(a)}{=} O\left(g^{(-1)}\left(\mathcal{W}_{\max }^{2}\right)\right) \\
& \stackrel{(b)}{=} O\left(g^{(-1)}(C(\mathrm{x}) / 2)\right)
\end{aligned}
$$

where (b) is from (37) and for (a) we prove the following technical proposition whose proof is presented in Appendix B.1.

Proposition 12 For $p \in(0,1)$,

$$
\sum_{k=1}^{\infty} g^{(-1)}(k) \cdot(1-p)^{k}=O\left(g^{(-1)}\left(p^{-2}\right)\right) .
$$

Combining (56), (57) and (58), the desired conclusion of Lemma 8 follows. This completes the proof of Lemma 8.

7. Proof of Lemma 6: $\boldsymbol{C}(\mathbf{x})<\boldsymbol{W}_{\text {max }}^{\mathbf{3}}$. We remind that the goal is to establish that starting with state $X(0)=\mathrm{x}=(\mathbf{Q}, \boldsymbol{\sigma}, \boldsymbol{a}, \boldsymbol{A}, \mathbf{B})$ such that $L(\mathrm{x})$ is large enough and $C(\mathrm{x})=\max \left\{g\left(\boldsymbol{A}_{\max }\right), \mathbf{B}_{\max }\right\}<\boldsymbol{W}_{\text {max }}^{3}$, after time

$$
h(\mathrm{x})=\frac{1}{2} \exp \left(\exp \left(\log ^{1 / 2} \boldsymbol{W}_{\max }\right)\right)
$$

the expected value of $L$ decreases by

$$
k(\mathrm{x})=\frac{\log ^{1 / 2} \boldsymbol{W}_{\max }}{2} \exp \left(\exp \left(\log ^{1 / 2} \boldsymbol{W}_{\max }\right)\right)=\log ^{1 / 2} \boldsymbol{W}_{\max } h(\mathrm{x}) .
$$


In other words,

$$
\mathbb{E}[L(h(\mathrm{x}))-L(0) \mid X(0)=\mathrm{x}] \leq-k(\mathrm{x}) .
$$

We note that for proving Lemma 6 , these will be the definition of functions $h$ and $k$ (cf. (33) and (34)) it concerns the case $C(\mathrm{x})<\boldsymbol{W}_{\max }^{3}$. To simplify notations, we will use notation $\mathbb{E}[\cdot]$ and $\mathbb{P}[\cdot]$ instead of $\mathbb{E}[\cdot \mid X(0)=\mathrm{x}]$ and $\mathbb{P}[\cdot \mid X(0)=\mathrm{x}]$ whenever clear from the context.

Similar to the proof of Lemma 6 for the case $C(\mathrm{x}) \geq \boldsymbol{W}_{\max }^{3}$ (presented in Section 6), we start by obtaining some bound for $\boldsymbol{W}_{\max }(\tau)$. To this end, note that if $L(\mathrm{x})$ is large enough, then either $\mathbf{Q}_{\max }, \boldsymbol{A}_{\max }$ or $\mathbf{B}_{\max }$ are large. Since $\mathbf{Q}_{\max }, \boldsymbol{A}_{\max }$ and $\mathbf{B}_{\max }$ are bounded in terms of $\boldsymbol{W}_{\max }, \boldsymbol{W}_{\max }$ is necessarily large if $L(\mathrm{x})$ is large enough. Now for large enough $L(\mathrm{x})$ and hence large enough $\boldsymbol{W}_{\max }$, we have

$$
\begin{aligned}
\boldsymbol{W}_{\max } & =\max \left\{\log \mathbf{Q}_{\max }, \exp \left(\sqrt{\log g\left(\boldsymbol{A}_{\max }\right)}\right)\right\} \\
& \stackrel{(a)}{<} \max \left\{\log \mathbf{Q}_{\max }, \exp \left(\sqrt{3 \log \boldsymbol{W}_{\max }}\right\}\right. \\
& \stackrel{(b)}{=} \log \mathbf{Q}_{\max },
\end{aligned}
$$

where (a) is from the condition $g\left(\boldsymbol{A}_{\max }\right) \leq C(\mathrm{x})<\boldsymbol{W}_{\max }^{3}$ and (b) is because $\boldsymbol{W}_{\max }>\exp \left(\sqrt{3 \log \boldsymbol{W}_{\max }}\right)$ for large enough $\boldsymbol{W}_{\max }$. Henceforth, we shall assume that

$$
\boldsymbol{W}_{\max }=\log \mathbf{Q}_{\max }
$$

and consequently $\mathbf{Q}_{\max }$ can be also assumed to be large enough if $L(\mathrm{x})$ is large. Using this, we obtain the following lower bound of $\boldsymbol{W}_{\max }(\tau)$ : for $\tau \leq h(\mathrm{x})$,

$$
\begin{aligned}
\boldsymbol{W}_{\max }(\tau) & \geq \log \mathbf{Q}_{\max }(\tau) \stackrel{(a)}{\geq} \log \left(\mathbf{Q}_{\max }-h(\mathbf{x})\right) \\
& \stackrel{(b)}{=} \log \left(\mathbf{Q}_{\max }-o\left(\mathbf{Q}_{\max }\right)\right) \\
& \stackrel{(c)}{\geq} \frac{1}{2} \log \mathbf{Q}_{\max }:=\mathcal{W}_{\min }
\end{aligned}
$$

where (a) is from 1-Lipschitz property of $\mathbf{Q}_{\max }(\cdot),(\mathrm{c})$ holds for large enough $\mathbf{Q}_{\max }$ and (b) is due to

$$
h(\mathrm{x})=\frac{1}{2} \exp \left(\exp \left(\log ^{1 / 2} \boldsymbol{W}_{\max }\right)\right)=\frac{1}{2} \exp \left(\exp \left(\log \log ^{1 / 2} \mathbf{Q}_{\max }\right)\right)=o\left(\mathbf{Q}_{\max }\right) .
$$


On the other hand, $\boldsymbol{W}_{\max }(\tau)$ can be upper bounded as follows: for $\tau \leq h(\mathrm{x})$,

$$
\begin{aligned}
\boldsymbol{W}_{\max }(\tau) & \leq \max \left\{\log \mathbf{Q}_{\max }(\tau), \exp \left(\sqrt{\log g\left(\boldsymbol{A}_{\max }(\tau)\right)}\right)\right\} \\
& \stackrel{(d)}{\leq} \max \left\{\log \left(\mathbf{Q}_{\max }+h(\mathbf{x})\right), \exp \left(\sqrt{\log g\left(\boldsymbol{A}_{\max }+h(\mathbf{x})\right)}\right)\right\} \\
& \stackrel{(e)}{\leq} \max \left\{\log \left(\mathbf{Q}_{\max }+o\left(\mathbf{Q}_{\max }\right)\right), \log \mathbf{Q}_{\max }\right\} \\
& \leq 2 \cdot \log \mathbf{Q}_{\max },
\end{aligned}
$$

where (d) is from 1-Lipschitz properties of $\mathbf{Q}_{\max }(\cdot), \boldsymbol{A}_{\max }(\cdot)$ and (e) follows from below using $\boldsymbol{A}_{\max } \leq g^{(-1)}(C(\mathrm{x})), C(\mathrm{x})<\boldsymbol{W}_{\max }$ :

$$
\begin{aligned}
\sqrt{\log g\left(\boldsymbol{A}_{\max }+h(\mathbf{x})\right)} & \leq \sqrt{\log g\left(g^{(-1)}(C(\mathbf{x}))+h(\mathbf{x})\right)} \\
& \leq \sqrt{\log g\left(g^{(-1)}\left(\boldsymbol{W}_{\max }^{3}\right)+h(\mathbf{x})\right)} \\
& =\sqrt{\log g\left(g^{(-1)}\left(\log ^{3} \mathbf{Q}_{\max }\right)+h(\mathbf{x})\right)} \\
& \stackrel{(f)}{\leq} \sqrt{\log g(2 \cdot h(\mathbf{x}))} \\
& =\sqrt{\log g\left(\exp \left(\exp \left(\log \log ^{1 / 2} \mathbf{Q}_{\max }\right)\right)\right.} \\
& =\log \log \mathbf{Q}_{\max } .
\end{aligned}
$$

In above, for (f) one can check

$$
g^{(-1)}\left(\log ^{3} \mathbf{Q}_{\max }\right) \leq h(\mathbf{x})=\frac{1}{2} \exp \left(\exp \left(\log \log ^{1 / 2} \mathbf{Q}_{\max }\right)\right)
$$

for large enough $\mathbf{Q}_{\max }$. Combining (61) and (62), it follows that for $\tau \leq h(\mathbf{x})$,

$$
\mathcal{W}_{\min } \leq \boldsymbol{W}_{\max }(\tau) \leq \mathcal{W}_{\max }
$$

where $\mathcal{W}_{\min }:=\frac{1}{2} \log \mathbf{Q}_{\max }$ and $\mathcal{W}_{\max }:=2 \log \mathbf{Q}_{\max }$.

Three Lemmas. Now we state the following key lemmas that will lead to (59). We shall present their proofs in Section 7.1, 7.2 and 7.3, respectively.

Lemma 13 Given initial state $X(0)=\mathrm{x}=(\mathbf{Q}, \boldsymbol{\sigma}, \boldsymbol{a}, \boldsymbol{A}, \mathbf{B})$, let $C(\mathrm{x})<$ $\boldsymbol{W}_{\max }^{3}, \boldsymbol{\lambda} \in \boldsymbol{\Lambda}$ and $\mathbf{Q}_{\max }$ be large enough. Then

$$
\mathbb{E}\left[\sum_{i} F\left(Q_{i}(h(\mathrm{x}))\right)\right] \leq \sum_{i} F\left(Q_{i}\right)-\Omega\left(\log \log \mathbf{Q}_{\max }\right) \cdot h(\mathbf{x}) .
$$


Lemma 14 Given initial state $X(0)=\mathrm{x}=(\mathbf{Q}, \boldsymbol{\sigma}, \boldsymbol{a}, \boldsymbol{A}, \mathbf{B})$, let $C(\mathrm{x})<$ $\boldsymbol{W}_{\max }^{3}$ and $\mathbf{Q}_{\max }$ be large enough. Then for any $i$ and $j \in \mathcal{N}(i)$

$$
\mathbb{E}\left[A_{j}^{i}(h(\mathrm{x}))^{2}\right]=O(h(\mathrm{x})) .
$$

Lemma 15 Given initial state $X(0)=\mathrm{x}=(\mathbf{Q}, \boldsymbol{\sigma}, \boldsymbol{a}, \boldsymbol{A}, \mathbf{B})$, let $C(\mathrm{x})<$ $\boldsymbol{W}_{\max }^{3}$ and $\mathbf{Q}_{\max }$ be large enough. Then for any $i$ and $j \in \mathcal{N}(i)$

$$
\mathbb{E}\left[g^{(-1)}\left(B_{j}^{i}(h(\mathbf{x}))\right)\right]=O\left(g^{(-1)}\left(4 \log ^{2} \mathbf{Q}_{\max }\right)\right) .
$$

Concluding (59) using Lemma 13, 14 and 15. These lemmas lead to the desired conclusion (59) as follows:

$$
\begin{aligned}
& \mathbb{E}[L(h(\mathrm{x}))-L(0)] \\
& =\mathbb{E}\left[\sum_{i} F\left(Q_{i}(h(\mathrm{x}))\right)-\sum_{i} F\left(Q_{i}\right)\right]+\mathbb{E}\left[\sum_{i, j} A_{j}^{i}(h(\mathrm{x}))^{2}-\left(A_{j}^{i}\right)^{2}\right] \\
& \quad+\mathbb{E}\left[\sum_{i, j} g^{(-1)}\left(B_{j}^{i}(h(\mathrm{x}))\right)-g^{(-1)}\left(B_{j}^{i}\right)\right] \\
& \quad-\Omega\left(\log \log \mathbf{Q}_{\max }\right) \cdot h(\mathrm{x})+O(h(\mathrm{x}))+O\left(g^{(-1)}\left(4 \log ^{2} \mathbf{Q}_{\max }\right)\right) \\
& \quad \stackrel{(a)}{\leq}-\Omega\left(\log \log \mathbf{Q}_{\max }\right) \cdot h(\mathrm{x}) \\
& =-\Omega\left(\log \boldsymbol{W}_{\max }\right) \cdot h(\mathrm{x}) \\
& \stackrel{(b)}{\leq}-\log ^{1 / 2} \boldsymbol{W}_{\max } \cdot h(\mathrm{x}),
\end{aligned}
$$

where (a) is because $g^{(-1)}\left(4 \log ^{2} \mathbf{Q}_{\max }\right)=o(h(\mathbf{x}))$ for our choice of $h(\mathbf{x})=$ $\frac{1}{2} \exp \left(\exp \left(\log \log ^{1 / 2} \mathbf{Q}_{\max }\right)\right)$ and (b) holds for large enough $\boldsymbol{W}_{\max }$. This completes the proof of Lemma 6 for the case $C(\mathrm{x})<\boldsymbol{W}_{\max }^{3}$. 
7.1. Proof of Lemma 13. We start by observing that

$$
\begin{aligned}
\mathbb{E}\left[\sum_{i} F\left(Q_{i}(h(\mathrm{x}))\right)-\sum_{i} F\left(Q_{i}\right)\right] \\
=\sum_{\tau=0}^{h(\mathrm{x})-1} \mathbb{E}\left[\sum_{i} F\left(Q_{i}(\tau+1)\right)-\sum_{i} F\left(Q_{i}(\tau)\right)\right] \\
=\sum_{\tau=0}^{h(\mathrm{x})-1} \sum_{i} \mathbb{E}\left[F\left(Q_{i}(\tau+1)\right)-F\left(Q_{i}(\tau)\right)\right] \\
\stackrel{(a)}{\leq} \sum_{\tau=0}^{h(\mathrm{x})-1} \sum_{i} \mathbb{E}\left[\left(Q_{i}(\tau+1)-Q_{i}(\tau)\right) \cdot f\left(Q_{i}(\tau+1)\right)\right] \\
=\sum_{\tau=0}^{h(\mathrm{x})-1} \sum_{i} \mathbb{E}\left[\left(Q_{i}(\tau+1)-Q_{i}(\tau)\right) \cdot f\left(Q_{i}(\tau)\right)\right]+O(h(\mathrm{x})),
\end{aligned}
$$

where (a) is due to the convexity of $F$ and the last inequality is from 1Lipschitz property of $Q_{i}(\cdot)$. For each term in the summation of (64), we consider the following.

$$
\begin{aligned}
& \mathbb{E}\left[\left(Q_{i}(\tau+1)-Q_{i}(\tau)\right) \cdot f\left(Q_{i}(\tau)\right)\right] \\
& \quad=\mathbb{E}\left[\left(\zeta_{i}(\tau)-\sigma_{i}(\tau) \mathbf{I}_{\left\{Q_{i}(\tau)>0\right\}}\right) \cdot f\left(Q_{i}(\tau)\right)\right] \\
& \quad \stackrel{(a)}{=} \mathbb{E}\left[\zeta_{i}(\tau) \cdot f\left(Q_{i}(\tau)\right)\right]-\mathbb{E}\left[\sigma_{i}(\tau) \cdot f\left(Q_{i}(\tau)\right)\right] \\
& \quad \stackrel{(b)}{=} \mathbb{E}\left[\lambda_{i} \cdot f\left(Q_{i}(\tau)\right)\right]-\mathbb{E}\left[\sigma_{i}(\tau) \cdot f\left(Q_{i}(\tau)\right)\right],
\end{aligned}
$$

where for (a) we use $\mathbf{I}_{\left\{Q_{i}(\tau)>0\right\}} \cdot f\left(Q_{i}(\tau)\right)=f\left(Q_{i}(\tau)\right)$ since $f(0)=0$; for (b) we use the fact that $\zeta_{i}(\tau), Q_{i}(\tau)$ are independent random variables and $\mathbb{E}\left[\zeta_{i}(\tau)\right]=\lambda_{i}$. Now from (64) and (65), it follows that

$$
\begin{aligned}
\mathbb{E}\left[\sum_{i} F\left(Q_{i}(h(\mathrm{x}))\right)-\sum_{i} F\left(Q_{i}\right)\right] \\
\quad=\sum_{\tau=0}^{h(\mathrm{x})-1} \sum_{i} \mathbb{E}\left[\left(Q_{i}(\tau+1)-Q_{i}(\tau)\right) \cdot f\left(Q_{i}(\tau)\right)\right]+O(h(\mathrm{x})) \\
\quad=\sum_{\tau=0}^{h(\mathrm{x})-1} \mathbb{E}\left[\sum_{i} \lambda_{i} \cdot f\left(Q_{i}(\tau)\right)-\sum_{i} \sigma_{i}(\tau) \cdot f\left(Q_{i}(\tau)\right)\right]+O(h(\mathrm{x})) \\
\quad \leq \sum_{\tau=0}^{h(\mathrm{x})-1} \mathbb{E}\left[(1-\varepsilon)\left(\max _{\boldsymbol{\rho} \in \mathcal{I}(G)} \boldsymbol{\rho} \cdot f(\mathbf{Q}(\tau))\right)-\boldsymbol{\sigma}(\tau) \cdot f(\mathbf{Q}(\tau))\right]+O(h(\mathrm{x}))
\end{aligned}
$$


where the last equality is from $\boldsymbol{\lambda}=\left[\lambda_{i}\right] \in \boldsymbol{\Lambda} \subset(1-\varepsilon) \operatorname{Conv}(\mathcal{I}(G))$ for some $\varepsilon>0$ and the convex hull $\operatorname{Conv}(\mathcal{I}(G))$ of $\mathcal{I}(G)$. Hence, for the proof of Lemma 13 , it is enough to prove that

$(66)$

$\sum_{\tau=0}^{h(\mathrm{x})-1} \mathbb{E}\left[(1-\varepsilon)\left(\max _{\boldsymbol{\rho} \in \mathcal{I}(G)} \boldsymbol{\rho} \cdot f(\mathbf{Q}(\tau))\right)-\boldsymbol{\sigma}(\tau) \cdot f(\mathbf{Q}(\tau))\right]=-\Omega\left(\log \log \mathbf{Q}_{\max }\right) \cdot h(\mathrm{x})$.

Further, it suffices to prove that for some $R=o(h(\mathrm{x}))$

(67)

$\sum_{\tau=R}^{h(\mathrm{x})-1} \mathbb{E}\left[(1-\varepsilon)\left(\max _{\boldsymbol{\rho} \in \mathcal{I}(G)} \boldsymbol{\rho} \cdot f(\mathbf{Q}(\tau))\right)-\boldsymbol{\sigma}(\tau) \cdot f(\mathbf{Q}(\tau))\right]=-\Omega\left(\log \log \mathbf{Q}_{\max }\right) \cdot(h(\mathrm{x})-R)$,

since the remaining terms in (66), other than those present in (67), are dominated by (67) as follows:

$$
\begin{aligned}
\sum_{\tau=0}^{R-1} \mathbb{E} & {\left[(1-\varepsilon)\left(\max _{\boldsymbol{\rho} \in \mathcal{I}(G)} \boldsymbol{\rho} \cdot f(\mathbf{Q}(\tau))\right)-\boldsymbol{\sigma}(\tau) \cdot f(\mathbf{Q}(\tau))\right] } \\
\leq & \sum_{\tau=0}^{R-1} \mathbb{E}\left[\max _{\boldsymbol{\rho} \in \mathcal{I}(G)} \boldsymbol{\rho} \cdot f(\mathbf{Q}(\tau))\right] \leq \sum_{\tau=0}^{R-1} \mathbb{E}\left[n \cdot f\left(\mathbf{Q}_{\max }(\tau)\right)\right] \\
\leq & \sum_{\tau=0}^{R-1} \mathbb{E}\left[n \cdot f\left(\exp \left(\boldsymbol{W}_{\max }(\tau)\right)\right)\right]=O(R) \cdot f\left(\exp \left(\mathcal{W}_{\max }\right)\right) \\
& =o(h(\mathrm{x})) \cdot \log \log \mathbf{Q}_{\max },
\end{aligned}
$$

where the last equality is from $f(x)=\log \log x, \mathcal{W}_{\max } \leq 2 \log \mathbf{Q}_{\max }$ (cf. (63)) and $R=o(h(\mathrm{x}))$.

Now we will proceed toward proving (67). Equivalently, we will find some $R=o(h(\mathrm{x}))$ such that for all $\tau \in[R, h(\mathrm{x})-1]$,

(68) $\mathbb{E}\left[(1-\varepsilon)\left(\max _{\boldsymbol{\rho} \in \mathcal{I}(G)} \boldsymbol{\rho} \cdot f(\mathbf{Q}(\tau))\right)-\boldsymbol{\sigma}(\tau) \cdot f(\mathbf{Q}(\tau))\right]=-\Omega\left(\log \log \mathbf{Q}_{\max }\right)$.

Three sub-Lemmas. The proof of (68) will be established as a consequence of following three lemmas. Their proof are presented in Section 7.1.1, 7.1.2 and 7.1.3, respectively.

Lemma A Let $\boldsymbol{\mu}(\tau)$ denote the distribution of $\{\boldsymbol{\sigma}(\tau), \boldsymbol{a}(\tau)\}$ at time $\tau$. Then,

$$
\left\|\boldsymbol{\mu}(\tau)-\boldsymbol{\delta}_{\{\boldsymbol{\sigma}, \boldsymbol{a}\}} \cdot P(0)^{\tau}\right\|_{T V} \leq O\left(\sum_{s=0}^{\tau-1} \mathbb{E}\left[\|P(s)-P(0)\|_{\infty}\right]\right),
$$


where $\boldsymbol{\delta}_{x}$ is the Dirac distribution of singleton support $x$ and $P(\tau)$ denotes the transition matrix for the Markov chain corresponding to the schedule $\boldsymbol{\sigma}(\tau)$, as described in Section 4.2 as well as Section 3 which is function of node weights $\boldsymbol{W}(\tau)$ determined by $\mathbf{Q}(\tau)$ and $\boldsymbol{A}(\tau)$ as per (1).

Lemma B Let event $\mathcal{E}_{\tau}$ at time $\tau$ be as follows:

$$
\begin{aligned}
\mathcal{E}_{\tau}:=\{X(\tau): & W_{i}(\tau) \geq \exp \left(\log \log ^{\eta} \mathbf{Q}_{\max }\right) \text { and } \\
& \left.g\left(A_{j}^{i}(\tau)\right) \leq \log ^{4} \mathbf{Q}_{\max } \text { for all } i, j \in \mathcal{N}(i)\right\},
\end{aligned}
$$

where $\eta:=1 / 4^{n}$. Then, there exists $R_{B}=\operatorname{polylog}\left(\mathbf{Q}_{\max }\right)$ such that for $\tau<h(\mathrm{x})$,

$$
\begin{array}{r}
(1-\varepsilon)\left(\max _{\boldsymbol{\rho} \in \mathcal{I}(G)} \boldsymbol{\rho} \cdot f(\mathbf{Q}(\tau))\right)-\mathbb{E}\left[\boldsymbol{\sigma}\left(\tau+R_{B}\right) \cdot f(\mathbf{Q}(\tau)) \mid X(\tau) \in \mathcal{E}_{\tau}\right] \\
\leq-\frac{\varepsilon}{4} \cdot \log \log \mathbf{Q}_{\max } .
\end{array}
$$

Lemma $\mathbf{C}$ There exists $R_{C}=o(h(\mathrm{x}))$ such that $\mathcal{E}_{\tau}$ happens with high probability for $\tau \in\left[R_{C}, h(\mathrm{x})\right]$ i.e.

$$
\mathbb{P}\left[\mathcal{E}_{\tau}\right]=1-o(1),
$$

where we recall $o(1)$ means that $o(1) \rightarrow 0$ as $\mathbf{Q}_{\max } \rightarrow \infty$. The $o(1)$ bound is uniform over all $\tau$.

Remarks for Lemma $A, B$ and $C$. Lemma A captures the evolution of the distribution of schedules. It is used crucially to establish Lemma B. Lemma B implies that (68) holds at time $\tau+R_{B}$ if $\mathcal{E}_{\tau}$ happens at time $\tau$ and $R_{B}$ is small enough to guarantee $f(\mathbf{Q}(\tau)) \approx f\left(\mathbf{Q}\left(\tau+R_{B}\right)\right)$. Lemma $\mathrm{C}$ indeed suggests that such event $\mathcal{E}_{\tau}$ happens with high probability. This will essentially lead to (68).

Concluding (68). We choose $R$ for (68) as

$$
R=R_{B}+R_{C} .
$$

It is easy to check $R=o(h(\mathrm{x}))$ since $R_{B}=\operatorname{polylog}\left(\mathbf{Q}_{\max }\right)=o(h(\mathrm{x}))$ and $R_{C}=o(h(\mathrm{x}))$. For $\tau \in[R, h(\mathrm{x})-1]$, we break the left hand side of (68) into 
two parts as follows:

$$
\begin{aligned}
\mathbb{E} & {\left[(1-\varepsilon)\left(\max _{\boldsymbol{\rho} \in \mathcal{I}(G)} \boldsymbol{\rho} \cdot f(\mathbf{Q}(\tau))\right)-\boldsymbol{\sigma}(\tau) \cdot f(\mathbf{Q}(\tau))\right] } \\
& =\mathbb{P}\left[\mathcal{E}_{\tau-R_{B}}\right] \cdot \mathbb{E}\left[(1-\varepsilon)\left(\max _{\boldsymbol{\rho} \in \mathcal{I}(G)} \boldsymbol{\rho} \cdot f(\mathbf{Q}(\tau))\right)-\boldsymbol{\sigma}(\tau) \cdot f(\mathbf{Q}(\tau)) \mid \mathcal{E}_{\tau-R_{B}}\right]
\end{aligned}
$$

$$
+\mathbb{P}\left[\mathcal{E}_{\tau-R_{B}}^{c}\right] \cdot \mathbb{E}\left[(1-\varepsilon)\left(\max _{\boldsymbol{\rho} \in \mathcal{I}(G)} \boldsymbol{\rho} \cdot f(\mathbf{Q}(\tau))\right)-\boldsymbol{\sigma}(\tau) \cdot f(\mathbf{Q}(\tau)) \mid \mathcal{E}_{\tau-R_{B}}^{c}\right] .
$$

For the first term in (69), we obtain

$$
\begin{gathered}
\mathbb{P}\left[\mathcal{E}_{\tau-R_{B}}\right] \cdot \mathbb{E}\left[(1-\varepsilon)\left(\max _{\boldsymbol{\rho} \in \mathcal{I}(G)} \boldsymbol{\rho} \cdot f(\mathbf{Q}(\tau))\right)-\boldsymbol{\sigma}(\tau) \cdot f(\mathbf{Q}(\tau)) \mid \mathcal{E}_{\tau-R_{B}}\right] \\
\stackrel{(a)}{=}(1-o(1)) \cdot \mathbb{E}\left[(1-\varepsilon)\left(\max _{\boldsymbol{\rho} \in \mathcal{I}(G)} \boldsymbol{\rho} \cdot f(\mathbf{Q}(\tau))\right)-\boldsymbol{\sigma}(\tau) \cdot f(\mathbf{Q}(\tau)) \mid \mathcal{E}_{\tau-R_{B}}\right] \\
\stackrel{(b)}{\leq}(1-o(1)) \cdot \mathbb{E}\left[(1-\varepsilon)\left(\max _{\boldsymbol{\rho} \in \mathcal{I}(G)} \boldsymbol{\rho} \cdot f\left(\mathbf{Q}\left(\tau-R_{B}\right)\right)\right)\right. \\
\left.\quad-\boldsymbol{\sigma}(\tau) \cdot f\left(\mathbf{Q}\left(\tau-R_{B}\right)\right) \mid \mathcal{E}_{\tau-R_{B}}\right]-O\left(f\left(R_{B}\right)\right) \\
\stackrel{(c)}{\leq}-(1-o(1)) \cdot \frac{\varepsilon}{4} \cdot \log \log \mathbf{Q}_{\max }-O\left(f\left(R_{B}\right)\right)
\end{gathered}
$$

$\stackrel{(d)}{\leq}-\frac{\varepsilon}{8} \cdot \log \log \mathbf{Q}_{\max }$,

where (a) and (c) are from Lemma C and Lemma B, respectively. For (b), we use 1-Lipschitz property of $Q_{i}(\cdot)$ and $|f(x)-f(y)|<f(|x-y|)+O(1)$ for $f(x)=\log \log x$. For $(\mathrm{d})$, we use

$$
f\left(R_{B}\right)=f\left(\operatorname{polylog}\left(\mathbf{Q}_{\max }\right)\right)=o\left(f\left(\mathbf{Q}_{\max }\right)\right)=o\left(\log \log \mathbf{Q}_{\max }\right) .
$$

For the second term in (69), we observe that

$$
\begin{aligned}
& \mathbb{P}\left[\mathcal{E}_{\tau-R_{B}}^{c}\right] \cdot \mathbb{E}\left[(1-\varepsilon)\left(\max _{\boldsymbol{\rho} \in \mathcal{I}(G)} \boldsymbol{\rho} \cdot f(\mathbf{Q}(\tau))\right)-\boldsymbol{\sigma}(\tau) \cdot f(\mathbf{Q}(\tau)) \mid \mathcal{E}_{\tau-R_{B}}^{c}\right] \\
& \quad \stackrel{(e)}{\leq} o(1) \cdot \mathbb{E}\left[\left(\max _{\boldsymbol{\rho} \in \mathcal{I}(G)} \boldsymbol{\rho} \cdot f(\mathbf{Q}(\tau))\right) \mid \mathcal{E}_{\tau-R_{B}}^{c}\right] \\
& \quad \leq o(1) \cdot \mathbb{E}\left[n \cdot f\left(Q_{\max }(\tau)\right) \mid \mathcal{E}_{\tau-R_{B}}^{c}\right] \\
& \quad \stackrel{(f)}{=} o(1) \cdot O\left(f\left(\mathbf{Q}_{\max }\right)\right)=o\left(\log \log \mathbf{Q}_{\max }\right),
\end{aligned}
$$


where (e) is from Lemma $\mathrm{C}$ and (f) is due to

$$
\begin{aligned}
f\left(\mathbf{Q}_{\max }(\tau)\right) & \leq f\left(\mathbf{Q}_{\max }+\tau\right) \leq f\left(\mathbf{Q}_{\max }+h(\mathbf{x})\right) \\
& =f\left(\mathbf{Q}_{\max }+o\left(\mathbf{Q}_{\max }\right)\right)=O\left(f\left(\mathbf{Q}_{\max }\right)\right) .
\end{aligned}
$$

Finally, combining (69), (70) and (71), the desired (68) follows as

$$
\mathbb{E}\left[(1-\varepsilon)\left(\max _{\boldsymbol{\rho} \in \mathcal{I}(G)} \boldsymbol{\rho} \cdot f(\mathbf{Q}(\tau))\right)-\boldsymbol{\sigma}(\tau) \cdot f(\mathbf{Q}(\tau))\right] \leq-\frac{\varepsilon}{16} \cdot \log \log \mathbf{Q}_{\max } .
$$

This completes the proof of Lemma 13.

7.1.1. Proof of Lemma A. Let $\boldsymbol{\mu}(\tau+1: \tau)$ be the distribution of $\{\boldsymbol{\sigma}(\tau+$ $1), \boldsymbol{a}(\tau+1)\}$ at time $\tau+1$ given network state $X(\tau)$ at time $\tau$. From the definition of $P(\tau)$, we have

$$
\boldsymbol{\mu}(\tau+1: \tau)=\boldsymbol{\delta}_{\{\boldsymbol{\sigma}(\tau), \boldsymbol{a}(\tau)\}} P(\tau),
$$

where we recall that $P(\tau)$ is a function of the network state $X(\tau)$ since node weights $\boldsymbol{W}(\tau)$ are decided by $\mathbf{Q}(\tau)$ and $\boldsymbol{A}(\tau)$ as per (1). By taking expectations on both sides of the above equation, we obtain

$$
\boldsymbol{\mu}(\tau+1)=\mathbb{E}\left[\boldsymbol{\delta}_{\{\boldsymbol{\sigma}(\tau), \boldsymbol{a}(\tau)\}} P(\tau)\right],
$$

where the expectation is with respect to the distribution of $X(\tau)$. Using the above relation, we have

$$
\begin{aligned}
\boldsymbol{\mu}(\tau+1) & =\mathbb{E}\left[\boldsymbol{\delta}_{\{\boldsymbol{\sigma}(\tau), \boldsymbol{a}(\tau)\}} P(\tau)\right] \\
& =\mathbb{E}\left[\mathbb{E}\left[\boldsymbol{\delta}_{\{\boldsymbol{\sigma}(\tau), \boldsymbol{a}(\tau)\}} P(\tau) \mid \mathbf{Q}(\tau), \boldsymbol{A}(\tau)\right]\right] \\
& =\mathbb{E}\left[\mathbb{E}\left[\boldsymbol{\delta}_{\{\boldsymbol{\sigma}(\tau), \boldsymbol{a}(\tau)\}} \mid \mathbf{Q}(\tau), \boldsymbol{A}(\tau)\right] \cdot P(\tau)\right] \\
& =\mathbb{E}[\tilde{\boldsymbol{\mu}}(\tau) \cdot P(\tau)],
\end{aligned}
$$

where the expectation is with respect to the distribution of $\{\mathbf{Q}(\tau), \boldsymbol{A}(\tau)\}$ and we have used notation

$$
\tilde{\boldsymbol{\mu}}(\tau)=\tilde{\boldsymbol{\mu}}(\mathbf{Q}(\tau), \boldsymbol{A}(\tau)):=\mathbb{E}\left[\boldsymbol{\delta}_{\{\boldsymbol{\sigma}(\tau), \boldsymbol{a}(\tau)\}} \mid \mathbf{Q}(\tau), \boldsymbol{A}(\tau)\right] .
$$

This leads to the following recursive relation between $\boldsymbol{\mu}(\tau+1)$ and $\boldsymbol{\mu}(\tau)$.

$$
\begin{aligned}
\boldsymbol{\mu}(\tau+1) & =\mathbb{E}[\tilde{\boldsymbol{\mu}}(\tau) \cdot P(\tau)] \\
& =\mathbb{E}[\tilde{\boldsymbol{\mu}}(\tau) \cdot P(0)]+\mathbb{E}[\tilde{\boldsymbol{\mu}}(\tau) \cdot(P(\tau)-P(0))] \\
& =\mathbb{E}[\tilde{\boldsymbol{\mu}}(\tau)] \cdot P(0)+e(\tau) \\
& =\boldsymbol{\mu}(\tau) \cdot P(0)+e(\tau),
\end{aligned}
$$


where we define

$$
e(\tau):=\mathbb{E}[\tilde{\boldsymbol{\mu}}(\tau) \cdot(P(\tau)-P(0))] .
$$

By recursive application of this relation, we obtain

$$
\begin{aligned}
\boldsymbol{\mu}(\tau) & =\boldsymbol{\mu}(0) \cdot P(0)^{\tau}+\sum_{s=0}^{\tau-1} e(\tau-1-s) \cdot P(0)^{s} \\
& =\boldsymbol{\delta}_{\{\boldsymbol{\sigma}, \boldsymbol{a}\}} \cdot P(0)^{\tau}+\sum_{s=0}^{\tau-1} e(s) \cdot P(0)^{\tau-1-s} .
\end{aligned}
$$

Now we obtain the desired conclusion of Lemma A from the above inequality as follows:

$$
\begin{aligned}
\left\|\boldsymbol{\mu}(\tau)-\boldsymbol{\delta}_{\{\boldsymbol{\sigma}, \boldsymbol{a}\}} \cdot P(0)^{\tau}\right\|_{T V} & =\left\|\sum_{s=0}^{\tau-1} e(s) \cdot P(0)^{\tau-1-s}\right\|_{T V} \\
& \leq \sum_{s=0}^{\tau-1}\left\|e(s) \cdot P(0)^{\tau-1-s}\right\|_{T V} \\
& \leq O\left(\sum_{s=0}^{\tau-1}\|e(s)\|_{T V}\right) \\
& \leq O\left(\sum_{s=0}^{\tau-1} \mathbb{E}\left[\|P(s)-P(0)\|_{\infty}\right]\right),
\end{aligned}
$$

where we have used the fact that $P(0)^{\tau-1-s}$ (resp. $\left.\tilde{\boldsymbol{\mu}}(\tau)\right)$ is a transition matrix (resp. distribution vector) of finite dimension, independent of initial state $\mathrm{x}$. This completes the proof of Lemma A.

7.1.2. Proof of Lemma B. Recall that $\tau$ is time such that event $\mathcal{E}_{\tau}$ holds, where

$$
\begin{aligned}
\mathcal{E}_{\tau}:=\{X(\tau): & W_{i}(\tau) \geq \exp \left(\log \log ^{\eta} \mathbf{Q}_{\max }\right) \text { and } \\
& \left.g\left(A_{j}^{i}(\tau)\right) \leq \log ^{4} \mathbf{Q}_{\max } \text { for all } i, j \in \mathcal{N}(i)\right\},
\end{aligned}
$$

with $\eta:=1 / 4^{n}$. We wish to show the existence of $R_{B}$ so that $R_{B}=$ $\operatorname{polylog}\left(\mathbf{Q}_{\max }\right)$ and

$$
\begin{array}{r}
(1-\varepsilon)\left(\max _{\boldsymbol{\rho} \in \mathcal{I}(G)} \boldsymbol{\rho} \cdot f(\mathbf{Q}(\tau))\right)-\mathbb{E}\left[\boldsymbol{\sigma}\left(\tau+R_{B}\right) \cdot f(\mathbf{Q}(\tau)) \mid X(\tau) \in \mathcal{E}_{\tau}\right] \\
\leq-\frac{\varepsilon}{4} \cdot \log \log \mathbf{Q}_{\max } .
\end{array}
$$


To that end, we shall show that the above property holds for

$$
R_{B}:=T_{\operatorname{mix}}\left(1 / \mathbf{Q}_{\max }, n, 2 \log \mathbf{Q}_{\max }\right),
$$

where $T_{\text {mix }}$ is defined as per (23) as part of the statement of Lemma 4 . Clearly, from definition $R_{B}=\operatorname{polylog}\left(\mathbf{Q}_{\max }\right)$. Now given network state $X(\tau) \in \mathcal{E}_{\tau}$ at time $\tau<h(\mathrm{x})$, we have

$$
\left\|\boldsymbol{\delta}_{\{\boldsymbol{\sigma}(\tau), \boldsymbol{a}(\tau)\}} \cdot P(\tau)^{R_{B}}-\boldsymbol{\pi}(\tau)\right\|_{T V} \leq \frac{1}{\mathbf{Q}_{\max }}=o(1),
$$

where we let $\boldsymbol{\pi}(\tau)$ denote the unique stationary distribution of $P(\tau)$ and use Lemma 4 with $W_{\max }(\tau) \leq \mathcal{W}_{\max }=2 \log \mathbf{Q}_{\max }$ (cf. (63)). The above equality suggests the following: for distribution $\boldsymbol{\mu}\left(\tau+R_{B}: \tau\right)$ of $\boldsymbol{\sigma}\left(\tau+R_{B}\right)$ given network state $X(\tau)$,

$$
\begin{aligned}
\| \boldsymbol{\mu}(\tau & \left.+R_{B}: \tau\right)-\boldsymbol{\pi}(\tau) \|_{T V} \\
& \leq\left\|\boldsymbol{\mu}\left(\tau+R_{B}: \tau\right)-\boldsymbol{\delta}_{\{\boldsymbol{\sigma}(\tau), \boldsymbol{a}(\tau)\}} \cdot P(\tau)^{R_{B}}\right\|_{T V} \\
& +\left\|\boldsymbol{\delta}_{\{\boldsymbol{\sigma}(\tau), \boldsymbol{a}(\tau)\}} \cdot P(\tau)^{R_{B}}-\boldsymbol{\pi}(\tau)\right\|_{T V} \\
& =O\left(\sum_{s=\tau}^{\tau+R_{B}-1} \mathbb{E}\left[\|P(s)-P(\tau)\|_{\infty}\right]\right)+o(1) \\
& \stackrel{(a)}{\leq} O\left(\sum_{s=\tau}^{\tau+R_{B}-1} \mathbb{E}\left[\max _{i}\left|W_{i}(s)-W_{i}(\tau)\right|\right]\right)+o(1) \\
& \stackrel{(b)}{\leq} \sum_{s=\tau}^{\tau+R_{B}-1} O\left(\frac{(s-\tau) \cdot 2 \exp \left(\log _{\log } \log ^{\eta} \mathbf{Q}_{\max }\right)}{g^{(-1)}\left(\exp \left(\log \log ^{2 \eta} \mathbf{Q}_{\max }\right)\right)}\right)+o(1) \\
& \stackrel{(c)}{\leq} \frac{\mathbf{p o l y l o g}\left(\mathbf{Q}_{\max }\right) \cdot 2 \exp \left(\log \log ^{\eta} \mathbf{Q}_{\max }\right)}{g^{(-1)}\left(\exp \left(\log \log ^{2 \eta} \mathbf{Q}_{\max }\right)\right)}+o(1) \\
& \stackrel{(d)}{=} o(1)+o(1)=o(1),
\end{aligned}
$$

where (a) is from Proposition 16 that is stated below (and the proof is presented in Appendix B.2); (b), (c) and (d) follow from the Corollary 22 in Appendix A; by definition $R_{B}=\operatorname{poly} \log \left(\mathbf{Q}_{\max }\right), W_{i}(\tau) \geq \exp \left(\log \log { }^{\eta} \mathbf{Q}_{\max }\right)$ due to event $\mathcal{E}_{\tau}$ and

$$
\frac{g^{(-1)}\left(\exp \left(\log \log ^{2 \eta} \mathbf{Q}_{\max }\right)\right)}{\exp \left(\log \log ^{\eta} \mathbf{Q}_{\max }\right)}=\operatorname{superpolylog}\left(\mathbf{Q}_{\max }\right) .
$$


Proposition 16 Given two weights $\boldsymbol{W}^{1}=\left[W_{i}^{1}\right]$ and $\boldsymbol{W}^{2}=\left[W_{i}^{2}\right]$, let $P^{1}$ and $P^{2}$ be the Markov chains (i.e. their transition matrices) on $\Omega$ we described in Section 4.2 using node weights $\boldsymbol{W}^{1}$ and $\boldsymbol{W}^{2}$, respectively. Then,

$$
\left|P_{x x^{\prime}}^{1}-P_{x x^{\prime}}^{2}\right|=O\left(\max _{i}\left|W_{i}^{1}-W_{i}^{2}\right|\right), \quad \text { for all } x, x^{\prime} \in \Omega .
$$

From the above inequality (72), it follows that

$$
\begin{aligned}
& \mathbb{E}\left[\boldsymbol{\sigma}\left(\tau+R_{B}\right) \cdot \log \boldsymbol{W}(\tau) \mid X(\tau) \in \mathcal{E}_{\tau}\right] \\
& \geq \mathbb{E}\left[\boldsymbol{\sigma}_{\boldsymbol{\pi}(\tau)} \cdot \log \boldsymbol{W}(\tau) \mid X(\tau) \in \mathcal{E}_{\tau}\right] \\
& \quad-\left\|\boldsymbol{\mu}\left(\tau+R_{B}: \tau\right)-\boldsymbol{\pi}(\tau)\right\|_{T V}\left(\max _{\boldsymbol{\rho} \in \mathcal{I}(G)} \boldsymbol{\rho} \cdot \log \boldsymbol{W}(\tau)\right) \\
& \quad \stackrel{(a)}{\geq}\left(1-\left\|\boldsymbol{\mu}\left(\tau+R_{B}: \tau\right)-\boldsymbol{\pi}(\tau)\right\|_{T V}\right)\left(\max _{\boldsymbol{\rho} \in \mathcal{I}(G)} \boldsymbol{\rho} \cdot \log \boldsymbol{W}(\tau)\right)-O(1) \\
& \quad=(1-o(1))\left(\max _{\boldsymbol{\rho} \in \mathcal{I}(G)} \boldsymbol{\rho} \cdot \log \boldsymbol{W}(\tau)\right)-O(1),
\end{aligned}
$$

where $\boldsymbol{\sigma}_{\boldsymbol{\pi}(\tau)} \in \mathcal{I}(G)$ is the random variable drawn by $\boldsymbol{\pi}(\tau)$ and for (a) we use the following proposition and the product-form characterization of $\boldsymbol{\pi}(\tau)$ in Lemma 2 (proof can be found in Appendix).

Proposition 17 (Gibbs' Maximal Principle) Let $T: \Omega \rightarrow \mathbb{R}$ and let $\mathcal{M}(\Omega)$ be space of all distributions on $\Omega$. Define $F: \mathcal{M}(\Omega) \rightarrow \mathbb{R}$ as

$$
F(\boldsymbol{\mu})=\mathbb{E}\left[T\left(x_{\boldsymbol{\mu}}\right)\right]+H_{E R}(\boldsymbol{\mu}),
$$

where $x_{\boldsymbol{\mu}} \in \Omega$ in the random variable drawn by $\boldsymbol{\mu}$ and $H_{E R}(\boldsymbol{\mu})$ is the standard discrete entropy of $\boldsymbol{\mu}$. Then, $F$ is uniquely maximized by the distribution $\boldsymbol{\nu}$, where

$$
\nu_{x}=\frac{1}{Z} \exp (T(x)), \quad \text { for any } x \in \Omega,
$$

where $Z$ is the normalization constant (or partition function). Further, with respect to $\boldsymbol{\nu}$, we have

$$
\mathbb{E}\left[T\left(x_{\boldsymbol{\nu}}\right)\right] \geq \max _{x \in \Omega} T(x)-\log |\Omega| .
$$


Concluding the proof of Lemma B. We further bound the difference between $f\left(Q_{i}(\tau)\right)$ and $\log W_{i}(\tau)$ as

$$
\begin{aligned}
\left|f\left(Q_{i}(\tau)\right)-\log W_{i}(\tau)\right| & =\left|f\left(Q_{i}(\tau)\right)-\max \left\{f\left(Q_{i}(\tau)\right), \max _{j \in \mathcal{N}(i)} \sqrt{\log g\left(A_{j}^{i}(\tau)\right)}\right\}\right| \\
& \leq \max _{j \in \mathcal{N}(i)} \sqrt{\log g\left(A_{j}^{i}(\tau)\right)} \\
& \stackrel{(a)}{\leq} \sqrt{\log \left(\log ^{4} \mathbf{Q}_{\max }\right)} \\
& =o\left(\log \log \mathbf{Q}_{\max }\right) \\
(74) & =o\left(f\left(\mathbf{Q}_{\max }\right)\right),
\end{aligned}
$$

where (a) is because $X(\tau) \in \mathcal{E}_{\tau}$. Hence, we have

$$
\begin{aligned}
\mathbb{E}\left[\boldsymbol{\sigma}\left(\tau+R_{B}\right) \cdot f(\mathbf{Q}(\tau)) \mid X(\tau) \in \mathcal{E}_{\tau}\right] \\
\quad \stackrel{(b)}{=} \mathbb{E}\left[\boldsymbol{\sigma}\left(\tau+R_{B}\right) \cdot \log \boldsymbol{W}(\tau) \mid X(\tau) \in \mathcal{E}_{\tau}\right]-o\left(f\left(\mathbf{Q}_{\max }\right)\right) \\
\quad \stackrel{(c)}{\geq}(1-o(1))\left(\max _{\boldsymbol{\rho} \in \mathcal{I}(G)} \boldsymbol{\rho} \cdot \log \boldsymbol{W}(\tau)\right)-O(1)-o\left(f\left(\mathbf{Q}_{\max }\right)\right) \\
\quad \stackrel{(d)}{\geq}(1-o(1))\left(\max _{\boldsymbol{\rho} \in \mathcal{I}(G)} \boldsymbol{\rho} \cdot f(\mathbf{Q}(\tau))\right)-o\left(f\left(\mathbf{Q}_{\max }\right)\right) \\
\quad \stackrel{(e)}{\geq}(1-o(1))\left(\max _{\boldsymbol{\rho} \in \mathcal{I}(G)} \boldsymbol{\rho} \cdot f(\mathbf{Q}(\tau))\right),
\end{aligned}
$$

where (b), (d) are from (74), (c) is due to (73) and (e) follows from

$$
\begin{aligned}
& \max _{\boldsymbol{\rho} \in \mathcal{I}(G)} \boldsymbol{\rho} \cdot f(\mathbf{Q}(\tau)) \geq f\left(\mathbf{Q}_{\max }(\tau)\right) \geq f\left(\mathbf{Q}_{\max }-\tau\right) \\
& \quad \geq f\left(\mathbf{Q}_{\max }-h(\mathrm{x})\right)=f\left(\mathbf{Q}_{\max }-o\left(\mathbf{Q}_{\max }\right)\right)=\frac{1}{2} f\left(\mathbf{Q}_{\max }\right)
\end{aligned}
$$

for large enough $\mathbf{Q}_{\max }$. Finally, we derive the desired conclusion of Lemma $\mathrm{B}$ as

$$
\begin{aligned}
& (1-\varepsilon) \max _{\boldsymbol{\rho} \in \mathcal{I}(G)} \boldsymbol{\rho} \cdot f(\mathbf{Q}(\tau))-\mathbb{E}\left[\boldsymbol{\sigma}\left(\tau+R_{B}\right) \cdot f(\mathbf{Q}(\tau)) \mid X(\tau) \in \mathcal{E}_{\tau}\right] \\
& \quad \leq-(\varepsilon-o(1))\left(\max _{\boldsymbol{\rho} \in \mathcal{I}(G)} \boldsymbol{\rho} \cdot f(\mathbf{Q}(\tau))\right) \leq-\frac{\varepsilon}{2} \cdot\left(\max _{\boldsymbol{\rho} \in \mathcal{I}(G)} \boldsymbol{\rho} \cdot f(\mathbf{Q}(\tau))\right) \\
& \quad \leq-\frac{\varepsilon}{2} \cdot f\left(\mathbf{Q}_{\max }(\tau)\right) \leq-\frac{\varepsilon}{4} \cdot f\left(\mathbf{Q}_{\max }\right),
\end{aligned}
$$

where the last inequality is from (75) i.e. $f\left(\mathbf{Q}_{\max }(\tau)\right) \geq f\left(\mathbf{Q}_{\max }\right) / 2$. This completes the proof of Lemma B. 
7.1.3. Proof of Lemma $C$. We wish to show that there exists $R_{C}=$ $o(h(\mathrm{x}))$ so that for any $\tau \in\left[R_{C}, h(\mathrm{x})\right]$, the event $\mathcal{E}_{\tau}$ holds. For this, it is sufficient to establish that for any $\tau \in\left[R_{C}, h(\mathrm{x})\right]$, the following holds:

$$
\begin{gathered}
\mathbb{P}\left[g\left(A_{j}^{i}(\tau)\right) \leq \log ^{4} \mathbf{Q}_{\max }\right]=1-o(1), \\
\mathbb{P}\left[W_{i}(\tau) \geq \exp \left(\log \log ^{\eta} \mathbf{Q}_{\max }\right)\right]=1-o(1),
\end{gathered}
$$

with the $o(1)$ being uniform over the choice of $\tau \in\left[R_{C}, h(\mathrm{x})\right]$. We introduce some notations first:

$$
\begin{gathered}
\mathcal{L}_{1}:=\exp \left(\log \log ^{1 / 4} \mathbf{Q}_{\max }\right) \quad \text { and } \quad \mathcal{L}_{k}:=\exp \left(\log ^{1 / 4} \mathcal{L}_{k-1}\right) \quad \text { for } k \geq 2 \\
\mathcal{T}_{k}:=\sum_{l=1}^{k} g^{(-1)}\left(\mathcal{L}_{l} / 20\right) \cdot \log ^{n+3} \mathbf{Q}_{\max } .
\end{gathered}
$$

It can be checked inductively that

$$
\mathcal{L}_{k}=\exp \left(\log \log ^{1 / 4^{k}} \mathbf{Q}_{\text {max }}\right) \quad \text { and } \quad \mathcal{L}_{n}=\exp \left(\log \log ^{\eta} \mathbf{Q}_{\text {max }}\right),
$$

where we recall that $\eta=1 / 4^{n}$. Next, we shall show that both (76) and (77) hold for the definition of $R_{C}$ as follows:

$$
R_{C}:=\mathcal{T}_{n-1}=\sum_{k=1}^{n-1} g^{(-1)}\left(\exp \left(\log \log ^{1 / 4^{k}} \mathbf{Q}_{\max }\right) / 20\right) \cdot \log ^{n+3} \mathbf{Q}_{\max }
$$

Observe that as per this definition, $R_{C}=o(h(\mathrm{x}))$. Now we establish that indeed (76) and (77) hold.

Proof of (76). Define $T_{0}=0<T_{1}<T_{2}<\ldots$ so that $T_{m}$ is the $m$ th time when $A_{j}^{i}(\cdot)$ is updated i.e. $B_{j}^{i}\left(T_{m}-1\right) \geq 2$ and $B_{j}^{i}\left(T_{m}\right)=0$. Define $\widehat{m}$ as

$$
\widehat{m}:=\inf \left\{m: B_{j}^{i}\left(T_{m}-1\right) \geq g(\gamma) \text { and } m>1\right\},
$$

where $\gamma=g^{(-1)}\left(\log ^{4} \mathbf{Q}_{\max }\right)-2$. In addition, note that

$$
\begin{aligned}
g\left(A_{j}^{i}\right) & \leq C(\mathbf{x}) \leq \boldsymbol{W}_{\max }^{3}=\log ^{3} \mathbf{Q}_{\max } \\
& <\log ^{4} \mathbf{Q}_{\max }-3=g(\gamma+2)-3<g(\gamma-1)
\end{aligned}
$$

for large enough $\mathbf{Q}_{\max }$ since for large values $\left|g^{\prime}(\cdot)\right| \ll 1$. Thus, $A_{j}^{i}<\gamma-1$.

Now if $A_{j}^{i}(\tau) \geq \gamma+2=g^{(-1)}\left(\log ^{4} \mathbf{Q}_{\max }\right)$, one can check that $T_{\widehat{m}} \leq \tau$ since there should be at least two updates before time $\tau$ which make $A_{j}^{i}(\cdot)$ 
increase beyond $x$. Otherwise, $A_{j}^{i}(\cdot)$ will remain less than $\gamma+1$ under the algorithm until time $\tau$ since $A_{j}^{i}<\gamma-1$ in the beginning. Therefore, we have

$$
\begin{aligned}
& \mathbb{P}\left[g\left(A_{j}^{i}(\tau)\right) \geq \log ^{4} \mathbf{Q}_{\max }\right]=\mathbb{P}\left[A_{j}^{i}(\tau) \geq g^{(-1)}\left(\log ^{4} \mathbf{Q}_{\max }\right)\right] \\
& \quad \leq \mathbb{P}\left[T_{\widehat{m}} \leq \tau\right]=\sum_{k=1}^{\tau} \mathbb{P}\left[T_{\widehat{m}}=k\right] \\
& \quad \stackrel{(a)}{\leq} \sum_{k=1}^{\tau} \mathbb{P}\left[a_{j}(s)=1 \text { for } s=k-2, \ldots, k-\lceil g(x)\rceil-1\right] \\
& \quad \stackrel{(b)}{\leq} \sum_{k=1}^{\tau}\left(1-\frac{1}{\mathcal{W}_{\max }}\right)^{\lceil g(x)\rceil-1} \leq \tau \cdot\left(1-\frac{1}{2 \log \mathbf{Q}_{\max }}\right)^{\lceil g(x)\rceil-1} \\
& \quad \stackrel{(c)}{\leq} O\left(\frac{\tau}{\mathbf{Q}_{\max }}\right) \stackrel{(d)}{=} O\left(\frac{h(\mathbf{x})}{\mathbf{Q}_{\max }}\right)=o(1),
\end{aligned}
$$

where for (a) we utilize the fact $\widehat{m}>1$; for (b) one can observe that under the algorithm the probability that some node $j$ keeps attempting to transmit consecutively (without stopping) for some time interval of length $y$ is at $\operatorname{most}\left(1-\frac{1}{\mathcal{W}_{\max }}\right)^{y-1} ;$ (c) is due to $x=g^{(-1)}\left(\log ^{4} \mathbf{Q}_{\max }\right)-2$; (d) is from $h(\mathbf{x})=o\left(\mathbf{Q}_{\max }\right)$. This completes the proof of (76).

Proof of (77). We shall utilize the following result crucially whose proof is presented in Appendix C.

Proposition 18 Consider $i \in V, j \in \mathcal{N}(i) \cup\{i\}, W>0$ and network state $X(\tau)=\{\mathbf{Q}(\tau), \boldsymbol{\sigma}(\tau)$,

$\boldsymbol{a}(\tau), \boldsymbol{A}(\tau), \mathbf{B}(\tau)\}$ at time $\tau \leq h(\mathrm{x})$. Suppose that $\mathbf{Q}_{\max }$ is large enough and

$$
W_{i}(\tau)>W \geq \exp \left(\log \log ^{\delta} \mathbf{Q}_{\max }\right) \quad \text { for some } \delta>0 \text {. }
$$

Then,

$$
\mathbb{P}\left[W_{j}(\tau+s) \geq \exp \left(\log ^{1 / 4} W\right)\right] \geq 1-o(1),
$$

where $s:=g^{(-1)}(W / 20) \cdot \log ^{n+3} \mathbf{Q}_{\max }$. Here o(1) is uniform over choice of any $\tau \leq h(\mathrm{x})$.

Let $i^{*} \in \arg \max _{i} Q_{i}(\tau)$. For any node $j$, one can construct a path $j_{1}=$ $i^{*}, j_{2}, \ldots, j_{n}=j$ of length $n$ by allowing repetition. We recall the definition of $\mathcal{L}_{k}$ and $\mathcal{T}_{k}$.

$\mathcal{L}_{1}=\exp \left(\log \log ^{1 / 4} \mathbf{Q}_{\text {max }}\right) \quad$ and $\quad \mathcal{L}_{k}=\exp \left(\log ^{1 / 4} \mathcal{L}_{k-1}\right) \quad$ for $k \geq 2$ 


$$
\mathcal{T}_{0}=0 \quad \text { and } \quad \mathcal{T}_{k}=\sum_{l=1}^{k} g^{(-1)}\left(\mathcal{L}_{l} / 20\right) \cdot \log ^{n+3} \mathbf{Q}_{\text {max }} .
$$

Also define $\mathfrak{E}_{k}$ as

$$
\mathfrak{E}_{k}:=\left\{X\left(\tau+\mathcal{T}_{k}\right): W_{j_{k+1}}\left(\tau+\mathcal{T}_{k}\right) \geq \mathcal{L}_{k+1}\right\} .
$$

Now consider the following proposition:

Proposition 19 For $k=1, \ldots, n-1$ and $\tau \leq h(\mathrm{x})-\mathcal{T}_{n-1}$,

$$
\mathbb{P}\left[\mathfrak{E}_{k} \mid \mathfrak{E}_{1}, \ldots, \mathfrak{E}_{k-1}\right] \geq 1-o(1)
$$

In above, o(1) is uniform over choice of $\tau \leq h(\mathrm{x})-\mathcal{T}_{n-1}$.

Proof. We will prove Proposition 19 by induction. The base case $k=1$ follows from

$$
\begin{aligned}
W_{j_{1}}(\tau) & =W_{i^{*}}(\tau) \geq \log Q_{i^{*}}(\tau) \geq \log \left(Q_{i^{*}}-\tau\right) \\
& \geq \log \left(\mathbf{Q}_{\max }-h(\mathbf{x})\right) \geq \log \left(\mathbf{Q}_{\max }-o\left(\mathbf{Q}_{\max }\right)\right) \\
& \geq \frac{1}{2} \log \mathbf{Q}_{\max } \geq \exp \left(\log \log ^{1 / 4} \mathbf{Q}_{\max }\right) \\
& =\mathcal{L}_{1}
\end{aligned}
$$

where inequalities hold for large enough $\mathbf{Q}_{\max }$. It is easy to establish the necessary induction step using Proposition 18 and $\mathcal{L}_{k}=\exp \left(\log \log ^{\delta} \mathbf{Q}_{\max }\right)$ with $\delta=1 / 4^{k}$. This completes the proof of Proposition 19 .

Proposition 19 implies that for $\tau \in\left[0, h(\mathrm{x})-R_{C}\right]$,

$$
\begin{aligned}
\mathbb{P}\left[W_{i}\left(\tau+R_{C}\right) \geq \exp \left(\log \log ^{\eta} \mathbf{Q}_{\max }\right)\right] & =\mathbb{P}\left[W_{j}\left(\tau+\mathcal{T}_{n-1}\right) \geq \mathcal{L}_{n}\right] \\
& =\mathbb{P}\left[W_{j_{n}}\left(\tau+\mathcal{T}_{n-1}\right) \geq \mathcal{L}_{n}\right] \\
& =\mathbb{P}\left[\mathfrak{E}_{n-1}\right] \\
& =\prod_{k=1}^{n-1} \mathbb{P}\left[\mathfrak{E}_{k} \mid \mathfrak{E}_{1}, \ldots, \mathfrak{E}_{k-1}\right] \\
& \geq(1-o(1))^{n-1} \\
& \geq 1-o(1) .
\end{aligned}
$$

Note that in above, $n$ is a constant and $o(1)$ is with respect to scaling of network state such as $\mathbf{Q}_{\max }$. This completes the proof of (77). 
7.2. Proof of Lemma 14. We first state the following key proposition for the proof of Lemma 14.

Proposition 20 If $C(\mathrm{x}) \leq \boldsymbol{W}_{\max }^{3}$, then

$$
\mathbb{P}\left[A_{j}^{i}(\tau)>k\right]=\exp \left(-\frac{g(k)}{\mathcal{W}_{\max }}\right) \cdot O(\tau),
$$

for $\tau \leq h(\mathrm{x})$ and $k>g^{(-1)}\left(\boldsymbol{W}_{\max }^{3}\right)$. Recall that $\mathcal{W}_{\max }$ is defined as per (63).

Proof. First note that it is enough to consider the case when $k$ is an integer. Let random time $T_{0}=0<T_{1}<T_{2}<\ldots$ such that $T_{m}$ is the $m$ th time when $A_{j}^{i}(\cdot)$ is updated i.e. $B_{j}^{i}\left(T_{m}-1\right) \geq 2$ and $B_{j}^{i}\left(T_{m}\right)=0$. We define

$$
\widehat{m}:=\inf \left\{m: B_{j}^{i}\left(T_{m}-1\right) \geq g(k-1) \text { and } m>1\right\} .
$$

Now observe that if $A_{j}^{i}(\tau)>k$, then

$$
A_{j}^{i}(\tau)>k>A_{j}^{i}
$$

since $A_{j}^{i} \leq g^{(-1)}(C(\mathrm{x})) \leq g^{(-1)}\left(\boldsymbol{W}_{\max }^{3}\right)<k$. Hence, if $A_{j}^{i}(\tau)>k, T_{\widehat{m}} \leq \tau$ since there should be at least two updates before time $\tau$ which make $A_{j}^{i}(\cdot)$ increase beyond $k-1$. Otherwise, $A_{j}^{i}(\cdot)$ should keep less than $k+1$ under the algorithm until time $\tau$. Using this observation, we have

$$
\begin{aligned}
\mathbb{P}\left[A_{j}^{i}(\tau) \geq k\right] & \leq \mathbb{P}\left[T_{\widehat{m}} \leq \tau\right] \\
& =\sum_{l=1}^{\tau} \mathbb{P}\left[T_{\widehat{m}}=l\right] \\
& \stackrel{(a)}{\leq} \sum_{l=1}^{\tau} \mathbb{P}\left[a_{j}(s)=1 \text { for } s=l-2, \ldots, l-g(k-1)-1\right] \\
& \stackrel{(b)}{\leq} \sum_{l=1}^{\tau}\left(1-\frac{1}{\mathcal{W}_{\max }}\right)^{g(k-1)-1} \\
& \leq \tau \cdot\left(1-\frac{1}{\mathcal{W}_{\max }}\right)^{g(k-1)-1} \\
& =\exp \left(-\frac{g(k)}{\mathcal{W}_{\max }}\right) \cdot O(\tau),
\end{aligned}
$$

where (a) is from $\widehat{m}>1$ and for (b) one can observe that under the algorithm the probability that some node $j$ keeps attempting to transmit consecutively (without stopping) for some time interval of length $y$ is at most $\left(1-\frac{1}{\mathcal{W}_{\max }}\right)^{y-1}$. This completes the proof of Proposition 20. 
Completing proof of Lemma 14. We derive the following inequalities.

$$
\begin{aligned}
& \mathbb{E}\left[A_{j}^{i}(h(\mathrm{x}))^{2}\right]=\sum_{k=1}^{\infty} \mathbb{P}\left[A_{j}^{i}(h(\mathrm{x}))=k\right] \cdot k^{2} \\
& \quad=\sum_{k=1}^{\sqrt{h(\mathrm{x})}-1} \mathbb{P}\left[A_{j}^{i}(h(\mathrm{x}))=k\right] \cdot k^{2}+\sum_{k=\sqrt{h(\mathrm{x})}}^{\infty} \mathbb{P}\left[A_{j}^{i}(h(\mathrm{x}))=k\right] \cdot k^{2} \\
& \leq h(\mathrm{x})+\sum_{k=\sqrt{h(\mathrm{x})}}^{\infty} \mathbb{P}\left[A_{j}^{i}(h(\mathrm{x}))=k\right] \cdot k^{2} \\
& \quad \stackrel{(a)}{\leq} h(\mathrm{x})+\sum_{k=\sqrt{h(\mathrm{x})}}^{\infty} \frac{1}{k^{4}} \cdot O(h(\mathrm{x})) \cdot k^{2} \\
& \leq h(\mathrm{x})+O(h(\mathrm{x})) \cdot \sum_{k=\sqrt{h(\mathrm{x})}}^{\infty} \frac{1}{k^{2}}=O(h(\mathrm{x}))
\end{aligned}
$$

where for (a) we use the following inequality:

$$
\mathbb{P}[A(h(\mathrm{x}))=k] \leq \frac{1}{k^{4}} \cdot O(h(\mathrm{x})), \quad \text { for } k \geq \sqrt{h(\mathrm{x})} .
$$

Hence, it is enough to show (79) to complete the proof of Lemma 14.

From Proposition 20, it suffices to prove that

$$
\exp \left(-\frac{g(k)}{\mathcal{W}_{\max }}\right) \leq \frac{1}{k^{4}}, \quad \text { for } k \geq \sqrt{h(\mathrm{x})} .
$$

By taking the double-logarithm (i.e. $\log \log$ ) on both sides of the above inequality and using $g(k)=\exp \left(\log \log ^{4} k\right)$, we have the equivalent inequality as

$$
\log \log ^{4} k-\log \mathcal{W}_{\max } \geq \log 4+\log \log k .
$$

One can check the above inequality holds if $\log \log ^{4} k \geq 2 \log \mathcal{W}_{\max }$ since $\mathcal{W}_{\max }$ is large enough. Equivalently, the desired condition for $k$ is

$$
k \geq \exp \left(\exp \left(\left(2 \log \mathcal{W}_{\max }\right)^{1 / 4}\right)\right) .
$$

Finally, $k \geq \sqrt{h(\mathrm{x})}$ satisfies the above condition since

$$
\begin{aligned}
\exp \left(\exp \left(\left(2 \log \mathcal{W}_{\max }\right)^{1 / 4}\right)\right) & =\exp \left(\exp \left(\Theta\left(\log \log ^{1 / 4} \mathbf{Q}_{\max }\right)\right)\right) \\
& \leq \frac{1}{\sqrt{2}} \cdot \exp \left(\frac{1}{2} \exp \left(\log \log ^{1 / 2} \mathbf{Q}_{\max }\right)\right) \\
& =\sqrt{h(\mathbf{x})},
\end{aligned}
$$


where the first inequality is from the definition of $\mathcal{W}_{\max }$ in (63) and the second inequality holds for large enough $\mathbf{Q}_{\max }$. This completes the proof of (79), hence the proof of Lemma 14.

7.3. Proof of Lemma 15. To begin with, we note that the proof of Lemma 15 is almost identical to that of Lemma 8 in Section 6.2. Let the random time $\tau^{*}=\inf \left\{\tau: a_{j}(\tau)=0\right\}$ i.e. the first time when $j$ does not attempt to transmit, and the event $\mathfrak{E}$ denotes $\tau^{*} \geq h(\mathrm{x})$. Hence, if $\mathfrak{E}$ happens, $B(h(\mathrm{x}))=$ $B+h(\mathrm{x})$ and

$\mathbb{P}[\mathfrak{E}] \leq \mathbb{P}[j$ attempts to transmit consecutively for time $\tau \in[0, h(\mathrm{x})-1]]$ $(80) \leq\left(1-\frac{1}{\mathcal{W}_{\max }}\right)^{h(\mathrm{x})-1}$,

where the last inequality holds because $W_{j}(\tau)$ is uniformly bounded from above by $\mathcal{W}_{\max }$.

On the other hand, if the event $\mathfrak{E}$ does not happen, $j$ stops its transmission before time $h(\mathrm{x})$, hence $B_{j}^{i}(\cdot)$ should set to 0 before time $h(\mathrm{x})$. Based on this observation and arguments similar to those used for establishing (80), we obtain

$$
\begin{aligned}
\mathbb{P}\left[B_{j}^{i}(h(\mathrm{x}))=k \mid \mathfrak{E}^{c}\right] & \leq \mathbb{P}[j \text { attempts to transmit consecutively } \\
& \leq\left(1-\frac{1}{\mathcal{W}_{\text {max }}}\right)^{k-1} .
\end{aligned}
$$

Further observe that

$$
\begin{aligned}
\mathbb{E}\left[g^{(-1)}(B(h(\mathrm{x})))\right]= & \mathbb{P}[\mathfrak{E}] \cdot \mathbb{E}\left[g^{(-1)}\left(B_{j}^{i}(h(\mathrm{x}))\right) \mid \mathfrak{E}\right] \\
& +\mathbb{P}\left[\mathfrak{E}^{c}\right] \cdot \mathbb{E}\left[g^{(-1)}\left(B_{j}^{i}(h(\mathrm{x}))\right) \mid \mathfrak{E}^{c}\right] \\
\leq \mathbb{P}[\mathfrak{E}] \cdot \mathbb{E}\left[g^{(-1)}\left(B_{j}^{i}(h(\mathrm{x}))\right) \mid \mathfrak{E}\right] & +\mathbb{E}\left[g^{(-1)}\left(B_{j}^{i}(h(\mathrm{x}))\right) \mid \mathfrak{E}^{c}\right] .
\end{aligned}
$$

For the first term in (82), we consider the following using (80).

$$
\begin{aligned}
\mathbb{P}[\mathfrak{E}] \cdot \mathbb{E}\left[g^{(-1)}\left(B_{j}^{i}(h(\mathrm{x}))\right) \mid \mathfrak{E}\right] & \leq\left(1-\frac{1}{\mathcal{W}_{\max }}\right)^{h(\mathrm{x})-1} \cdot g^{(-1)}\left(B_{j}^{i}+h(\mathrm{x})\right) \\
& \stackrel{(a)}{\leq}\left(1-\frac{1}{h(\mathrm{x})}\right)^{h(\mathrm{x})-1} \cdot g^{(-1)}(h(\mathrm{x})+h(\mathrm{x})) \\
& \stackrel{(b)}{=} o(1),
\end{aligned}
$$


where (a) follows from $\mathcal{W}_{\max } \leq 2 \log \mathbf{Q}_{\max } \leq h(\mathrm{x})$ and $B_{j}^{i} \leq C(\mathrm{x}) \leq \boldsymbol{W}_{\max }^{3}=$ $\log ^{3} \mathbf{Q}_{\max } \leq h(\mathbf{x})$; one can check (b) for large enough $h(\mathbf{x})$.

For the second term in (82), we consider the following using (81).

$$
\begin{aligned}
\mathbb{E}\left[g^{(-1)}\left(B_{j}^{i}(h(\mathrm{x}))\right) \mid \mathfrak{E}^{c}\right] & \leq \sum_{k=1}^{\infty} g^{(-1)}(k) \cdot\left(1-\frac{1}{\mathcal{W}_{\max }}\right)^{k-1} \\
& \stackrel{(a)}{=} O\left(g^{(-1)}\left(\mathcal{W}_{\max }^{2}\right)\right) \\
& \leq O\left(g^{(-1)}\left(4 \log ^{2} \mathbf{Q}_{\max }\right)\right),
\end{aligned}
$$

where (a) is from Proposition 12. Combining (82), (83) and (84) completes the proof of Lemma 15.

8. Discussion. As the main result of this paper, we presented a new medium-access algorithm for an arbitrary wireless network where simultaneously transmitting nodes must form an independent set of the network graph. The algorithm is optimal in the sense that network Markov chain is positive-recurrent as long as the imposed traffic demand can be satisfied by some scheduling algorithm. The algorithm is entirely distributed: the only information it utilizes is its own queue-size and the history of collisions or successful transmissions. In a sense, this work settles an important question that has been of interest in distributed computation, communication, probability and learning.

The algorithm we presented builds upon the work of [18] where the algorithm required a bit of information exchange between neighbors per unit time. Specifically, the key technical contribution of our work is to get rid of this requirement by means of designing a novel learning mechanism that essentially estimates the rate of a Bernoulli process with time varying rates. To incorporate the new mechanism well with the prior algorithm in [18], in essence we analyzed the positive recurrence of coupled Markov chains (via their mixing properties): the first and second components are from scheduling and queueing dynamics, respectively. We believe that our method could be of much broader interest to establish the positive recurrence of such coupled Markov chains in general.

\section{REFERENCES}

[1] N. Abramson and F. Kuo (Editors). The aloha system. Computer-Communication Networks, 1973.

[2] D. J. Aldous. Ultimate instability of exponential back-off protocol for acknowledgement-based transmission control of random access communication channels. IEEE Transactions on Information Theory, 33(2):219-223, 1987. 
[3] V. Anantharam and P. Tsoucas. A proof of the Markov chain tree theorem. Statistics E Probability Letters, 8(2):189-192, June 1989.

[4] M. Dyer, A. Frieze, and R. Kannan. A random polynomial-time algorithm for approximating the volume of convex bodies. J. ACM, 38(1):1-17, 1991.

[5] S. Foss and T. Konstantopoulos. An overview of some stochastic stability methods. Journal of Operations Research, Society of Japan, 47(4), 2004.

[6] H. O. Georgii. Gibbs measures and phase transitions. Walter de Gruyter, 1988.

[7] L. A. Goldberg and P. D. MacKenzie. Analysis of practical backoff protocols for contention resolution with multiple servers. Journal of Computer and System Sciences, 58(1):232-258, 1999. Preliminary version in SODA '96: Proceedings of the seventh annual ACM-SIAM Symposium on Discrete Algorithms.

[8] L. A. Goldberg. Design and analysis of contention-resolution protocols, epsrc research grant gr/160982. http://www.csc.liv.ac.uk/ leslie/contention.html, Last updated, Oct. 2002.

[9] J. Hastad, T. Leighton, and B. Rogoff. Analysis of backoff protocols for multiple access channels. SIAM Journal on Computing, 25(4), 1996. Preliminary version in STOC '87: Proceedings of the nineteenth annual ACM Symposium on Theory of Computing.

[10] L. Jiang, D. Shah, J. Shin, and J. Walrand. Distributed random access algorithm: Scheduling and congesion control. IEEE Transactions on Information Theory, 56(12):6182-6207, 2010.

[11] L. Jiang and J. Walrand. A distributed csma algorithm for throughput and utility maximization in wireless networks. In Proceedings of 46 th Allerton Conference on Communication, Control, and Computing, Urbana-Champaign, IL, 2008.

[12] F. P. Kelly. Stochastic models of computer communication systems. J. R. Statist. Soc B, 47(3):379-395, 1985.

[13] F. P. Kelly and I. M. MacPhee. The number of packets transmitted by collision detect random access schemes. The Annals of Probability, 15(4):1557-1568, 1987.

[14] I. M. MacPhee. On optimal strategies in stochastic decision processes, d. phil. thesis, University of Cambridge, 1989.

[15] R. Metcalfe and D. Boggs. Distributed packet switching for local computer networks. Comm. ACM, 19:395-404, 1976.

[16] R. Montenegro and P. Tetali. Mathematical aspects of mixing times in Markov chains. Found. Trends Theor. Comput. Sci., 1(3):237-354, 2006.

[17] J. Mosely and P. A. Humblet. A class of efficient contention resolution algorithms for multiple access channels. IEEE Transactions on Communications, 33(2):145-151, 1985.

[18] S. Rajagopalan, D. Shah, and J. Shin. Network adiabatic theorem: an efficient randomized protocol for contention resolution. In Proceedings of the eleventh international joint conference on Measurement and modeling of computer systems, 133-144. ACM New York, NY, USA, 2009.

[19] D. Shah and J. Shin. Randomized scheduling algorithm for queueing networks. Annals of Applied Probability, 22(1):128-171, 2012.

[20] D. Shah and D. J. Wischik. Optimal scheduling algorithm for input queued switch. In Proceeding of IEEE INFOCOM, 2006.

[21] A. Sinclair. Algorithms for Random Generation and Counting: A Markov Chain Approach. Birkhäuser, Boston, 1993. 
[22] A. L. Stolyar. MaxWeight scheduling in a generalized switch: State space collapse and workload minimization in heavy traffic. Annals of Applied Probability, 14(1):1-53, 2004.

[23] B. S. Tsybakov and N. B. Likhanov. Upper bound on the capacity of a random multiple-access system. Problemy Peredachi Informatsii, 23(3):64-78, 1987.

\section{APPENDIX A: PROPERTIES OF $\boldsymbol{W}(\cdot)$}

Here we establish deterministic properties of $\boldsymbol{W}(\cdot)$ under the algorithm that will be useful to prove the main theorem in this paper. Specifically we establish that, for any $i, W_{i}(\cdot)$ changes slowly if it becomes large.

Proposition 21 There exists an absolute constant $w_{o} \geq 0$ so that for any node $i$ and time $\tau$, if $W_{i}(\tau) \geq w_{o}$ then

$$
\left|W_{i}(\tau+1)-W_{i}(\tau)\right| \leq \frac{W_{i}(\tau)}{g^{(-1)}\left(\exp \left(\log ^{2} W_{i}(\tau)\right)\right)} .
$$

Proof. We shall establish existence of large enough constant $w_{o}$ under which the claimed result holds. To that end, given node $i$ and time $\tau$, from definition of weight as per (1),

$$
W_{i}(\tau)=\exp \left(\max \left\{f\left(Q_{i}(\tau)\right), \sqrt{\max _{j \in \mathcal{N}(i)} \log g\left(A_{j}^{i}(\tau)\right)}\right\}\right),
$$

where we use $f(x)=\log \log x$. Now $Q_{i}(\cdot)$ and $A_{j}^{i}(\cdot)$ for any $j \in \mathcal{N}(i)$ change by at most \pm 1 in unit time. That is, they are uniformly 1-Lipschitz. Therefore,

$$
\begin{aligned}
& W_{i}(\tau+1) \leq \exp \left(\max \left\{f\left(Q_{i}(\tau)+1\right), \sqrt{\max _{j \in \mathcal{N}(i)} \log g\left(A_{j}^{i}(\tau)+1\right)}\right\}\right), \\
& W_{i}(\tau+1) \geq \exp \left(\max \left\{f\left(Q_{i}(\tau)-1\right), \sqrt{\max _{j \in \mathcal{N}(i)} \log g\left(A_{j}^{i}(\tau)-1\right)}\right\}\right) .
\end{aligned}
$$

Using (86), we shall establish an upper bound on $W_{i}(\tau+1)-W_{i}(\tau)$. To that end, consider $\exp \left(f\left(Q_{i}(\tau)+1\right)\right)$ : using Taylor's expansion

$$
\begin{aligned}
\exp \left(f\left(Q_{i}(\tau)+1\right)\right) & \leq \log \left(Q_{i}(\tau)+1\right) \\
& \leq \log Q_{i}(\tau)+\frac{1}{Q_{i}(\tau)} \\
& \stackrel{(a)}{\leq} W_{i}(\tau)+\frac{1}{\exp \left(W_{i}(\tau)\right)},
\end{aligned}
$$

where (a) follows from the fact that $\log y+1 / y \geq \log x+1 / x$ if $0<x \leq y$ for all $y$ large enough; $w_{o}$ is chosen so that such is true when $y \geq w_{o}$; the fact 
that $\log Q_{i}(\tau) \leq W_{i}(\tau)$ and the assumption that $W_{i}(\tau) \geq w_{o}$. In a similar manner, using Taylor's expansion and the form of the derivative of function $\exp (\sqrt{\log g(\cdot)})$, we have

$\exp \left(\sqrt{\log g\left(A_{j}^{i}(\tau)+1\right)}\right) \leq \exp \left(\sqrt{\log g\left(A_{j}^{i}(\tau)\right)}\right)\left[1+\frac{1}{A_{j}^{i}(\tau)} \frac{2 \log \log A_{j}^{i}(\tau)}{\log A_{j}^{i}(\tau)}\right]$

$$
\stackrel{(a)}{\leq} W_{i}(\tau)+W_{i}(\tau) \frac{1}{g^{(-1)}\left(\exp \left(\log ^{2} W_{i}(\tau)\right)\right)} .
$$

In above (a) follows because for all large enough $y, q(x) \leq q(y)$ for $0<x \leq y$ with $q(x)=\frac{1}{y} \frac{2 \log \log x}{\log x}$; for $y$ large enough $q(y) \leq \frac{1}{y} ; w_{o}$ is large enough so that these two inequalities are satisfied; $W_{i}(\tau) \geq \exp \left(\sqrt{\log A_{j}^{i}(\tau)}\right)$ and $W_{i}(\tau) \geq w_{o}$. From (86), (88) and (89), it follows that

$$
\begin{aligned}
W_{i}(\tau+1) & \leq W_{i}(\tau)+\max \left\{\exp \left(-W_{i}(\tau)\right), W_{i}(\tau) \frac{1}{g^{(-1)}\left(\exp \left(\log ^{2} W_{i}(\tau)\right)\right)}\right\} \\
& \leq W_{i}(\tau)+W_{i}(\tau) \frac{1}{g^{(-1)}\left(\exp \left(\log ^{2} W_{i}(\tau)\right)\right)}
\end{aligned}
$$

where the last inequality follows from the fact that for $x$ large enough, $\exp (-x) \leq x / g^{(-1)}\left(\exp \left(\log ^{2} x\right)\right)$. This is because $g^{(-1)}(y)=\exp \left(\exp \left(\log ^{1 / 4} y\right)\right)$,

$$
\begin{aligned}
\log ^{1 / 4} \exp \left(\log ^{2} x\right) & =o(\log x), \quad \text { and hence } \\
g^{(-1)}\left(\exp \left(\log ^{2} x\right)\right) & =\exp \left(\exp \left(\log ^{1 / 4}\left(\exp \left(\log ^{2} x\right)\right)\right)\right)=\exp (o(x)) .
\end{aligned}
$$

We shall assume that $w_{o}$ is large enough to satisfy this and $W_{i}(\tau) \geq w_{o}$.

This completes the proof of upper bound on $W_{i}(\tau+1)$ as desired by Proposition of 21 . In the process, we implicitly defined $w_{o}$ : it is a constant large enough so that (i) $\log y+1 / y \geq \log x+1 / x$ if $0<x \leq y$ for all $y \geq w_{o}$; (ii) $q(x) \leq q(y) \leq 1 / y$ for $0<x \leq y$ with $q(x)=\frac{1}{y} \frac{2 \log \log x}{\log x}$ for all $y \geq w_{o}$; and (iii) $\exp (-x) \leq x / g^{(-1)}\left(\exp \left(\log ^{2} x\right)\right)$ for all $x \geq w_{o}$. In a similar manner (details are skipped here), an appropriate lower bound on $W_{i}(\tau+1)$ can be obtained (which will lead to additional constraints on $w_{o}$ ). This completes the proof of Proposition 21.

Following is an immediate corollary of Proposition 21. 
Corollary 22 There exists a large enough constant $w_{1}$ so that for any node $i$, if $W_{i}(0) \geq w_{1}$ then

$$
\left|W_{i}(\tau)-W_{i}(0)\right| \leq \frac{2 W_{i}(0)}{g^{(-1)}\left(\exp \left(\log ^{2} W_{i}(0)\right)\right)} \tau,
$$

for $\tau \leq g^{(-1)}\left(\exp \left(\log ^{2} W_{i}(0)\right)\right) / 2 W_{i}(0)$. Subsequently, for any $w_{2} \geq w_{1}$,

$$
\begin{aligned}
& W_{i}(\tau) \leq w_{2}+1, \quad \text { if } \quad W_{i}(0) \leq w_{2}, \\
& W_{i}(\tau) \geq w_{2}-1, \quad \text { if } \quad W_{i}(0) \geq w_{2},
\end{aligned}
$$

for $2 \tau \leq g^{(-1)}\left(\exp \left(\log ^{2} w_{2}\right)\right) / w_{2}$.

Proof. We need to establish existence of large enough constant $w_{1}$ so that claimed result holds. For this, we start by constraining $w_{1} \geq w_{o}$, where $w_{o}$ is the constant from Proposition 21. In addition, we shall assume that $w_{1}$ is large enough so that for all $y \geq w_{1}, g^{(-1)}\left(\exp \left(\log ^{2} x\right)\right) / x \leq g^{(-1)}\left(\exp \left(\log ^{2} y\right)\right) / y$ as long as $0<x \leq y$. Such is a possibility since $g^{(-1)}\left(\exp \left(\log ^{2} x\right)\right)$ scales much faster than $x$. Further, function $g^{(-1)}\left(\exp \left(\log ^{2} x\right)\right) / x=\exp (o(x))$. Therefore, it can be shown that for large enough $x$,

$$
1 \ll \frac{g^{(-1)}\left(\exp \left(\log ^{2} x\right)\right)}{2 x} \leq \frac{g^{(-1)}\left(\exp \left(\log ^{2}(x-1)\right)\right)}{x-1} .
$$

We shall assume $w_{1}$ is chosen to be such large enough constant. Now applying Proposition 21, starting with $W_{i}(0) \geq w_{1}$, it follows that

$$
\left|W_{i}(1)-W_{i}(0)\right| \leq \frac{W_{i}(0)}{g^{(-1)}\left(\exp \left(\log ^{2} W_{i}(0)\right)\right)} .
$$

As per the above bound and choice of $w_{1},\left|W_{i}(1)-W_{i}(0)\right| \ll 1$. By repeated application of Proposition 21 till the summation of the right hand side of the above bound remains less than 1 , we obtain

$$
\left|W_{i}(\tau)-W_{i}(0)\right| \leq \sum_{s=0}^{\tau-1} \frac{W_{i}(s)}{g^{(-1)}\left(\exp \left(\log ^{2} W_{i}(s)\right)\right)} .
$$

Now for all such $s$, since $\left|W_{i}(s)-W_{i}(0)\right| \leq 1, W_{i}(0) \geq w_{1}$ and above discussed 
properties of $w_{1}, g^{(-1)}$ we obtain that for all such $\tau$

$$
\begin{aligned}
\left|W_{i}(\tau)-W_{i}(0)\right| & \leq \tau \frac{W_{i}(0)-1}{g^{(-1)}\left(\exp \left(\log ^{2}\left(W_{i}(0)-1\right)\right)\right)} \\
& \leq \tau \frac{2 W_{i}(0)}{g^{(-1)}\left(\exp \left(\log ^{2} W_{i}(0)\right)\right)} .
\end{aligned}
$$

Therefore, it follows that the above holds true for all $\tau$ such that

$$
\tau \leq \frac{g^{(-1)}\left(\exp \left(\log ^{2} W_{i}(0)\right)\right)}{2 W_{i}(0)} .
$$

The remaining consequences (90) and (91) follow immediately from this. This completes the proof of Corollary 22.

\section{APPENDIX B: PROOFS OF AUXILIARY PROPOSITIONS}

B.1. Proof of Proposition 12. Using elementary calculus, it follows that

$$
\lim _{x \rightarrow \infty} \frac{g^{(-1)}(x)}{\exp (\sqrt{x} / 8)}=0 \quad \text { and } \quad \lim _{x \rightarrow \infty} \frac{x g^{(-1)}\left(x^{2} / 4\right)}{g^{(-1)}\left(x^{2}\right)}=0 .
$$

Hence, there exists a constant $C_{1}>0$ such that for $x>C_{1}$,

$$
g^{(-1)}(x) \leq \exp (\sqrt{x} / 8) \quad \text { and } \quad x \cdot g^{(-1)}\left(x^{2} / 4\right) \leq g^{(-1)}\left(x^{2}\right) .
$$

If $k \geq p^{-2} / 4$, it follows that

$g^{(-1)}(k) \leq \exp (\sqrt{k} / 8) \leq \exp (p \cdot k / 4)=(\exp (p / 4))^{k} \leq(1+p / 2)^{k}$, 
where the last inequality holds from $\exp (x) \leq 1+2 x$ for $x \in(0,1)$. Using this,

$$
\begin{aligned}
\sum_{k=1}^{\infty} g^{(-1)}(k) \cdot(1-p)^{k} & \\
\quad & =\sum_{k=1}^{p^{-2} / 4} g^{(-1)}(k) \cdot(1-p)^{k}+\sum_{k=p^{-2} / 4+1}^{\infty} g^{(-1)}(k) \cdot(1-p)^{k} \\
& \stackrel{(a)}{=} O\left(g^{(-1)}\left(p^{-2} / 4\right) \cdot p^{-1}\right)+\sum_{k=p^{-2} / 4+1}^{\infty}(1+p / 2)^{k} \cdot(1-p)^{k} \\
& =O\left(g^{(-1)}\left(p^{-2} / 4\right) \cdot p^{-1}\right)+\sum_{k=p^{-2} / 4+1}^{\infty}(1-p / 2)^{k} \\
& =O\left(g^{(-1)}\left(p^{-2} / 4\right) \cdot p^{-1}\right)+O\left(p^{-1}\right) \\
& \stackrel{(b)}{=} O\left(g^{(-1)}\left(p^{-2}\right)\right),
\end{aligned}
$$

where (a) is from (93) and for (b) we use (92) under assuming $p^{-1}>C_{1}$. Otherwise, note that (b) is trivial since $p^{-1}$ bounded above by constant $C_{1}$. This completes the proof of Proposition 12.

B.2. Proof of Proposition 16. We recall the formula (13).

$$
P_{x x^{\prime}}=c\left(x, x^{\prime}\right) \cdot \prod_{i \in \boldsymbol{\sigma} \backslash \boldsymbol{\sigma}^{\prime}} \frac{1}{W_{i}} \cdot \prod_{i \in \boldsymbol{\sigma} \cap \boldsymbol{\sigma}^{\prime}}\left(1-\frac{1}{W_{i}}\right),
$$

where $c\left(x, x^{\prime}\right)$ is some constant independent of $\boldsymbol{W}=\left[W_{i}\right]$. Hence, we will consider $P_{x x^{\prime}}$ as a real-valued function in several variables $\left\{W_{i}\right\}$ i.e. $P_{x x^{\prime}}=$ $P_{x x^{\prime}}(\boldsymbol{W})$.

Now from the mean value theorem in several variables,

$$
\begin{aligned}
\left|P_{x x^{\prime}}^{1}-P_{x x^{\prime}}^{2}\right| & =\left|\nabla P_{x x^{\prime}}(\cdot) \cdot\left(\boldsymbol{W}^{1}-\boldsymbol{W}^{2}\right)\right| \\
& \leq\left\|\nabla P_{x x^{\prime}}(\cdot)\right\|_{2} \cdot\left\|\boldsymbol{W}^{1}-\boldsymbol{W}^{2}\right\|_{2} .
\end{aligned}
$$

Using this and (13), the desired conclusion follows since one can easily check that $\left\|\nabla P_{x x^{\prime}}(\cdot)\right\|_{2}=O(1)$ since each component of $\boldsymbol{W}$ is always at least 1 ; $\left\|\boldsymbol{W}^{1}-\boldsymbol{W}^{2}\right\|_{2}=O\left(\max _{i}\left|W_{i}^{1}-W_{i}^{2}\right|\right)$. 
B.3. Proof of Proposition 17. Observe that the definition of distribution $\boldsymbol{\nu}$ implies that for any $x \in \Omega$,

$$
T(x)=\log Z+\log \nu_{x} .
$$

Using this, for any distribution $\boldsymbol{\mu}$ on $\Omega$, we obtain

$$
\begin{aligned}
F(\boldsymbol{\mu}) & =\sum_{x} \mu_{x} T(x)-\sum_{x} \mu_{x} \log \mu_{x} \\
& =\sum_{x} \mu_{x}\left(\log Z+\log \nu_{x}\right)-\sum_{x} \mu_{x} \log \mu_{x} \\
& =\sum_{x} \mu_{x} \log Z+\sum_{x} \mu_{x} \log \frac{\nu_{x}}{\mu_{x}} \\
& =\log Z+\sum_{x} \mu_{x} \log \frac{\nu_{x}}{\mu_{x}} \\
& \leq \log Z+\log \left(\sum_{x} \mu_{x} \frac{\nu_{x}}{\mu_{x}}\right) \\
& =\log Z
\end{aligned}
$$

with equality if and only if $\boldsymbol{\mu}=\boldsymbol{\nu}$. To complete other claim of proposition, consider $x^{*} \in \arg \max T(x)$. Let $\boldsymbol{\mu}$ be the Dirac distribution $\boldsymbol{\delta}_{x^{*}}$. Then, for this distribution

$$
F(\boldsymbol{\mu})=T\left(x^{*}\right) .
$$

But, $F(\boldsymbol{\nu}) \geq F(\boldsymbol{\mu})$. Also, the maximal entropy of any distribution on $\Omega$ is $\log |\Omega|$. Therefore,

$$
\begin{aligned}
T\left(x^{*}\right) & \leq F(\boldsymbol{\nu}) \\
& =\mathbb{E}\left[T\left(x_{\boldsymbol{\nu}}\right)\right]+H_{E R}(\boldsymbol{\nu}) \\
& \leq \mathbb{E}\left[T\left(x_{\boldsymbol{\nu}}\right)\right]+\log |\Omega| .
\end{aligned}
$$

Re-arrangement of terms in (94) will imply the second claim of Proposition 17. This completes the proof of Proposition 17.

\section{APPENDIX C: PROOF OF PROPOSITION 18}

Recall that the Proposition 18 assumes that $C(\mathrm{x}) \leq \boldsymbol{W}_{\max }^{3}$. As per the statement of Proposition 18, we wish to prove that

$$
\mathbb{P}\left[W_{j}(\tau+s) \geq \exp \left(\log ^{1 / 4} W\right)\right] \geq 1-o(1),
$$


for choice of $s$ such that

$$
\begin{aligned}
s & =g^{(-1)}(W / 20) \cdot \log ^{n+3} \mathbf{Q}_{\max } \\
& \stackrel{(a)}{\leq} g^{(-1)}(W / 20) \cdot \exp \left((n+3) \log ^{1 / \delta} W\right) \\
& \stackrel{(b)}{\leq} \frac{g^{(-1)}\left(\exp \left(\log ^{2} W\right)\right)}{2 W},
\end{aligned}
$$

where (a) is from the condition $W \geq \exp \left(\log \log ^{\delta} \mathbf{Q}_{\max }\right)$ and one can check (b) for large enough $W$ (depending on $\delta$ ).

Now for the case of $j=i$, from above and Corollary 22, we have

$$
W_{j}(\tau+s) \geq W-1 \geq \exp \left(\log ^{1 / 4} W\right), \quad \text { with probability } 1,
$$

where the last inequality holds for large enough $W$.

Now consider the case $j \neq i$. In this case, we have

$$
\begin{aligned}
& \mathbb{P}\left[W_{j}(\tau+s) \geq \exp \left(\log ^{1 / 4} W\right)\right] \\
& \quad \stackrel{(a)}{\geq}\left[\operatorname{Pexp}\left(\sqrt{\log g\left(A_{i}^{j}(\tau+s)\right)}\right) \geq \exp \left(\log ^{1 / 4} W\right)\right] \\
& \quad=\mathbb{P}\left[g\left(A_{i}^{j}(\tau+s)\right) \geq \exp \left(\log ^{1 / 2} W\right)\right] \\
& \quad \stackrel{(b)}{\geq} \mathbb{P}\left[g\left(A_{i}^{j}(\tau+s)\right) \geq W / 20\right] \\
& \quad \stackrel{(c)}{\geq} 1-o(1),
\end{aligned}
$$

where (a) is from definition of $W_{j}$; for (b) we use $\exp \left(\log ^{1 / 2} W\right)<W / 20$ for large enough $W$; (c) is due to the following lemma. This completes the proof of Proposition 18.

Lemma 23 Consider given $i, j \in \mathcal{N}(i), W>0$ and network state $X(\tau)=$ $\{\mathbf{Q}(\tau), \boldsymbol{\sigma}(\tau), \boldsymbol{a}(\tau), \boldsymbol{A}(\tau), \mathbf{B}(\tau)\}$ at time $\tau \leq h(\mathbf{x})$. Suppose that $\mathbf{Q}_{\max }$ is large enough and

$$
W_{i}(\tau)>W \geq \exp \left(\log \log ^{\delta} \mathbf{Q}_{\max }\right) \quad \text { for some } \delta>0 .
$$

Then,

$$
\mathbb{P}\left[g\left(A_{i}^{j}(\tau+s)\right) \geq W / 20\right] \geq 1-o(1),
$$

where $s=g^{(-1)}(W / 20) \cdot \log ^{n+3} \mathbf{Q}_{\max }$. 
Proof. First consider the case when $g\left(A_{i}^{j}(\tau)\right)>W / 10$. 1-Lipschitz property of $A_{i}^{j}(\cdot)$ implies that

(96) $g\left(A_{i}^{j}\left(\tau+\tau^{\prime}\right)\right) \geq W / 20, \quad$ for all $\tau^{\prime} \leq g^{(-1)}(W / 10)-g^{(-1)}(W / 20)$.

On the other hand, we have

$$
\begin{aligned}
\frac{g^{(-1)}(W / 10)}{g^{(-1)}(W / 20)} & =\exp \left(\exp \left(\log ^{1 / 4} \frac{W}{10}\right)-\exp \left(\log ^{1 / 4} \frac{W}{20}\right)\right) \\
& \stackrel{(a)}{\geq} \exp \left(\exp \left(\log ^{1 / 4} \frac{W}{10}\right) \cdot \frac{1}{4} \log ^{-3 / 4} \frac{W}{20}\right) \\
& \stackrel{(b)}{=} \operatorname{superpolylog}\left(\mathbf{Q}_{\max }\right) .
\end{aligned}
$$

where for (a) we use $d(x)-d(x / 2) \geq d^{\prime}(x / 2) \cdot x / 2$ with $d(x)=\exp \left(\log ^{1 / 4} x\right)$ and $x=W / 10$; (b) is due to $W \geq \exp \left(\log \log ^{\delta} \mathbf{Q}_{\max }\right)$. Therefore, it follows that

$$
g\left(A_{i}^{j}(\tau+s)\right) \geq W / 20
$$

since

(98) $s=g^{(-1)}(W / 20) \cdot \operatorname{polylog}\left(\mathbf{Q}_{\max }\right) \ll g^{(-1)}(W / 10)-g^{(-1)}(W / 20)$,

where the inequality is from (96), (97), and large enough $\mathbf{Q}_{\max }$.

Now consider the second case when $g\left(A_{i}^{j}(\tau)\right) \leq W / 10$. As the first step, we will find some absolute upper and lower bounds on $W_{i}\left(\tau+\tau^{\prime}\right)$ and $g\left(A_{i}^{j}(\tau+\right.$ $\left.\tau^{\prime}\right)$ ) for $\tau^{\prime} \leq s$. Based on these bounds, we will construct a martingale with respect to $g\left(A_{i}^{j}(\cdot)\right)$ to control $g\left(A_{i}^{j}(\tau+\Delta)\right)$, which is indeed similar to the strategy we use for the proof of Lemma 7 in Section 6.1.

First step: Bounds for $W_{i}\left(\tau+\tau^{\prime}\right), g\left(A_{i}^{j}\left(\tau+\tau^{\prime}\right)\right)$. From Corollary 22, we observe that for $\tau^{\prime} \leq s$

$$
W_{i}\left(\tau+\tau^{\prime}\right) \geq W-1
$$

since using (98) it is easy to check that

$$
\tau^{\prime} \leq s \leq g^{(-1)}(W / 10) \leq \frac{g^{(-1)}\left(\exp \left(\log ^{2} W\right)\right)}{2 W} \quad \text { for large enough } W .
$$

For the bound of $g\left(A_{i}^{j}\left(\tau+\tau^{\prime}\right)\right)$, we obtain that for $\tau^{\prime} \leq s$

$$
g\left(A_{i}^{j}\left(\tau+\tau^{\prime}\right)\right) \leq W / 5,
$$


using 1-Lipschitz property of $A_{i}^{j}(\cdot)$ and

$$
\tau^{\prime} \leq s \stackrel{(a)}{\leq} g^{(-1)}(W / 10) \stackrel{(b)}{\leq} g^{(-1)}(W / 5)-g^{(-1)}(W / 10),
$$

where (a) is from (98) and (b) is due to $g^{(-1)}(x) \geq g^{(-1)}(x / 2) \cdot 2$ for large enough $x$.

Second step: Martingale construction. This part of the proof is similar to that stated in Section 6.1. We consider a modified network Markov chain where all the Markovian rules are same as the original chain except for $\boldsymbol{W}\left(\tau^{\prime}\right)=\boldsymbol{W}\left(\tau^{\prime}-1\right)$ for $\tau^{\prime}>\tau+s$ i.e. $\boldsymbol{W}(\cdot)$ is fixed after time $\tau+s$. This modification does not affect the distribution of $g\left(A_{i}^{j}(\tau+s)\right)$; it is merely for guaranteeing (99) all $\tau \geq 0$.

Now define random time $T_{0}=\tau<T_{1}<T_{2}<\ldots$ such that $T_{m}$ is the $m$ th time when $A_{i}^{j}(\cdot)$ is updated since time $\tau$ i.e. $B_{i}^{j}\left(T_{m}-1\right) \geq 2$ and $B_{i}^{j}\left(T_{m}\right)=0$. Further, define for $m \geq 0$,

$$
Y_{m}:=\left\{\begin{array}{ll}
\exp \left(g^{(-1)}(W)-A_{i}^{j}\left(T_{m}\right)\right) & \text { if } T_{m-1} \leq \tau+s \text { or } m=0 \\
\alpha \cdot Y_{m-1} & \text { otherwise }
\end{array},\right.
$$

where $\alpha=\frac{e}{4}+\frac{3}{4 e} \in(0,1)$. We shall establish that for all $m \geq 1$,

$$
\mathbb{E}\left[Y_{m+1} \mid \mathcal{F}_{m}\right] \leq \alpha \cdot Y_{m},
$$

where $\mathcal{F}_{m}$ denotes the filtration containing $Y_{k}, T_{k}$ for $0 \leq k \leq m$. Note that (101) is trivial if $T_{m}>\tau+s$ by definition of $Y_{m}$. When $T_{m} \leq \tau+s$, we observe that

$$
\begin{aligned}
\mathbb{E} & {\left[Y_{m+1} \mid \mathcal{F}_{m}\right] } \\
& =\mathbb{E}\left[\exp \left(g^{(-1)}(W)-A_{i}^{j}\left(T_{m+1}\right)\right) \mid \mathcal{F}_{m}\right] \\
& \stackrel{(a)}{\leq} \frac{3}{4} \cdot \exp \left(g^{(-1)}(W)-A_{i}^{j}\left(T_{m}\right)-1\right)+\frac{1}{4} \cdot \exp \left(g^{(-1)}(W)-A_{i}^{j}\left(T_{m}\right)+1\right) \\
& =\alpha \cdot \exp \left(g^{(-1)}(W)-A_{i}^{j}\left(T_{m}\right)\right) \\
& =\alpha \cdot Y_{m}
\end{aligned}
$$


where for (a) we use

$$
\begin{aligned}
& \mathbb{P}\left[A_{i}^{j}\left(T_{m+1}\right)=A_{i}^{j}\left(T_{m}\right)+1\right]=\mathbb{P}\left[B_{i}^{j}\left(T_{m}-1\right) \geq g\left(A_{i}^{j}\left(T_{m-1}\right)\right)\right] \\
& \quad \stackrel{(b)}{\geq}\left[B_{i}^{j}\left(T_{m}-1\right) \geq W / 5\right]=1-\mathbb{P}\left[B_{i}^{j}\left(T_{m}-1\right)<W / 5\right] \\
& \quad \geq 1-\sum_{k=1}^{W / 5} \mathbb{P}\left[B_{i}^{j}\left(T_{m}-1\right)=k\right] \\
& \quad \geq 1-\sum_{k=1}^{W / 5} \mathbb{P}\left[i \text { stops to attempt at time } T_{m}-1\right] \\
& \quad \stackrel{(c)}{\geq} 1-\sum_{k=1}^{W / 5} \frac{1}{W-1} \geq \frac{3}{4}
\end{aligned}
$$

where (b) and (c) are from (100) and (99), respectively.

From (101), $\left\{Z_{m}:=Y_{m} / \alpha^{m-1}, m \geq 1\right\}$ becomes a sub-martingale with respect to $\mathcal{F}_{m}$. If we define a stopping time $m^{*}$ as $m^{*}=\inf \left\{m: T_{m}>\tau+s\right\}$,

$$
\mathbb{E}\left[Z_{m^{*}}\right] \stackrel{(a)}{\leq} \mathbb{E}\left[Z_{1}\right]=\mathbb{E}\left[Y_{1}\right] \stackrel{(b)}{\leq} Y_{0} \cdot e=\exp \left(g^{(-1)}(W)-A_{i}^{j}(\tau)+1\right),
$$

where (a) and (b) are from the Doob's optional stopping theorem and 1Lipschitz property of $A_{i}^{j}(\cdot)$. Using the above inequality and Markov's inequality, we have

$$
\frac{Y_{m^{*}}}{\alpha^{m^{*}-1}}=Z_{m^{*}} \leq \exp \left(g^{(-1)}(W)-A_{i}^{j}(\tau)+1\right) \cdot \log \mathbf{Q}_{\max },
$$

$$
\text { with probability at least } 1-\frac{1}{\log \mathbf{Q}_{\max }}=1-o(1) \text {. }
$$

Finally, it follows that

$$
\begin{aligned}
A_{i}^{j}(\tau+s) & =A_{i}^{j}\left(m^{*}-1\right) \geq A_{i}^{j}\left(m^{*}\right)-1 \\
& =g^{(-1)}(W)-\left(g^{(-1)}(W)-A_{i}^{j}\left(T_{m^{*}}\right)\right)-1 \\
& =g^{(-1)}(W)-\log Y_{m^{*}}-1 \\
& \stackrel{(a)}{\geq} g^{(-1)}(W)-\left(g^{(-1)}(W)-A_{i}^{j}(\tau)+1\right. \\
& \left.\quad+\log \log \mathbf{Q}_{\max }-\left(m^{*}-1\right) \log \frac{1}{\alpha}\right)-1 \\
& \stackrel{(b)}{\geq} A_{i}^{j}(\tau)+\frac{s}{\log ^{n+2} \mathbf{Q}_{\max }} \log \frac{1}{\alpha}-\log \log \mathbf{Q}_{\max }-2 \\
& \stackrel{(c)}{\geq} g^{(-1)}(W / 20),
\end{aligned}
$$


where (c) is due to the choice of $s=g^{(-1)}(W / 20) \cdot \log ^{n+3} \mathbf{Q}_{\max }$ and large enough $\mathbf{Q}_{\max }$; (a) and (b) hold with probability 1-o(1) from (102) and the Proposition 24, respectively. This completes the proof of Lemma 23.

\section{Proposition 24}

$$
\mathbb{P}\left[m^{*}-1 \geq \frac{s}{\log ^{n+2} \mathbf{Q}_{\max }}\right]=1-o(1) .
$$

Proof. We start by defining random variable $U_{\tau^{\prime}}$.

$$
U_{\tau^{\prime}}=\left\{\begin{array}{ll}
1 & \text { if } A_{i}^{j}(\cdot) \text { is updated at time } \tau^{\prime} \\
0 & \text { otherwise }
\end{array}, \quad \text { for } \tau^{\prime} \in[\tau+1, \tau+s] .\right.
$$

In other words, $U_{\tau^{\prime}}=1$ only if $B_{i}^{j}\left(\tau^{\prime}-1\right) \geq 2$ and $B_{i}^{j}\left(\tau^{\prime}\right)=0$. By definition of $U_{\tau^{\prime}}$ and $m^{*}$,

$$
m^{*}-1=\sum_{\tau^{\prime}=\tau+1}^{\tau+s} U_{\tau^{\prime}}
$$

Since $W_{\max }\left(\tau^{\prime}\right) \leq \mathcal{W}_{\max }=O\left(\log \mathbf{Q}_{\max }\right)$ for $\tau^{\prime} \leq \tau+s$ (cf. (63)), the same arguments as in the proof of Proposition 11 lead to the following bound for the expectation of $m^{*}-1$.

$$
\mathbb{E}\left[m^{*}-1\right]=\mathbb{E}\left[\sum_{\tau^{\prime}=\tau+1}^{\tau+s} U_{\tau^{\prime}}\right]=\Omega\left(\frac{s}{\left(\mathcal{W}_{\max }\right)^{n+1}}\right)=\Omega\left(\frac{s}{\log ^{n+1} \mathbf{Q}_{\max }}\right) .
$$

Now we define random variable $Z_{\tau^{\prime}}$ as

$$
Z_{\tau^{\prime}}=\mathbb{E}\left[\sum_{\tau^{\prime}=\tau+1}^{\tau+s} U_{\tau^{\prime}} \mid U_{\tau+1}, \ldots, U_{\tau^{\prime}-1}\right]
$$

where $\tau^{\prime} \in[\tau+1, \tau+s+1]$. Hence, it is easy to observe that

$$
Z_{\tau+1}=\mathbb{E}\left[m^{*}-1\right] \quad \text { and } \quad Z_{\tau+s+1}=m^{*}-1 .
$$

Further, $\left\{Z_{\tau^{\prime}}: \tau^{\prime} \in[\tau+1, \tau+s+1]\right\}$ forms a martingale with bounded increments i.e. $\left|Z_{\tau^{\prime}}-Z_{\tau^{\prime}+1}\right| \leq 1$. Therefore, the statement of Proposition 24 follows by applying the Azuma's inequality to the martingale $\left\{Z_{\tau^{\prime}}\right\}$. 